AperTO - Archivio Istituzionale Open Access dell'Università di Torino

Colorectal cancer residual disease at maximal response to EGFR blockade displays a druggable Paneth cell-like phenotype

This is a pre print version of the following article:

Original Citation:

Availability:

This version is available http://hdl.handle.net/2318/1750197

since 2020-08-17T12:03:14Z

Published version:

DOI:10.1126/scitransImed.aax8313

Terms of use:

Open Access

Anyone can freely access the full text of works made available as "Open Access". Works made available under a Creative Commons license can be used according to the terms and conditions of said license. Use of all other works requires consent of the right holder (author or publisher) if not exempted from copyright protection by the applicable law. 


\title{
Colorectal cancer residual disease at maximal response to EGFR blockade displays a druggable Paneth cell-like phenotype
}

\author{
Barbara Lupo ${ }^{1,2}$ *, Francesco Sassi ${ }^{2}$, Marika Pinnelli ${ }^{1,2}$, Francesco Galimi $i^{1,2}$, \\ Eugenia R. Zanella ${ }^{2}$, Valentina Vurchio ${ }^{1,2}$, Giorgia Migliardi ${ }^{1,2}$, Paolo Armando Gagliardi ${ }^{1,2} \S$, \\ Alberto Puliafito ${ }^{1,2}$, Daria Manganaro ${ }^{3}$, Paolo Luraghi2\#, Michael Kragh ${ }^{4 \wedge}$, Mikkel W. Pedersen ${ }^{4}$, \\ Ivan D. Horak ${ }^{40}$, Carla Boccaccio ${ }^{1,2}$, Enzo Medico ${ }^{1,2}$, Luca Primo ${ }^{1,2}$, Daniel Nichol ${ }^{5}$, \\ Inmaculada Spiteri ${ }^{5}$, Timon Heide ${ }^{5}$, Alexandra Vatsiou ${ }^{5}$, Trevor A. Graham ${ }^{6}$, Elena Élez ${ }^{7}$, \\ Guillem Argiles ${ }^{7}$, Paolo Nuciforo ${ }^{7}$, Andrea Sottoriva ${ }^{5}$, Rodrigo Dienstmann, Diego Pasini ${ }^{3,8}$, \\ Elena Grassi ${ }^{2}$, Claudio Isella ${ }^{1,2}$, Andrea Bertotti ${ }^{1,2}{ }^{1 \ddagger}$, and Livio Trusolino ${ }^{1,2} \dagger \neq \#$ \\ ${ }^{1}$ Department of Oncology, University of Torino, 10060 Candiolo, Torino, Italy. \\ ${ }^{2}$ Candiolo Cancer Institute - FPO IRCCS, 10060 Candiolo, Torino, Italy. \\ ${ }^{3}$ European Institute of Oncology, 20139 Milano, Italy. \\ ${ }^{4}$ Symphogen A/S, 2750 Ballerup, Denmark \\ ${ }^{5}$ The Institute of Cancer Research, London SW7 3RP, United Kingdom \\ ${ }^{6}$ Centre for Genomics and Computational Biology, Barts Cancer Institute, Barts and the London \\ School of Medicine and Dentistry, Queen Mary University of London, London EC1M 6BQ, \\ United Kingdom \\ 7Vall d'Hebron Institute of Oncology (VHIO), 08035 Barcelona, Spain \\ ${ }^{8}$ Department of Health Sciences, University of Milano, 20142 Milano, Italy
}

* Barbara Lupo and Francesco Sassi contributed equally to this work

${ }^{\dagger}$ Andrea Bertotti and Livio Trusolino contributed jointly supervised this work

‡ Corresponding authors. E-mail: livio.trusolino@ircc.it (L.T.); andrea.bertotti@ircc.it (A.B.)

$\S$ Present address: Institute of Cell Biology, University of Bern, 3012 Bern, Switzerland

\# Present address: IFOM - The FIRC Institute of Molecular Oncology, 20139 Milano, Italy

^Present address: Novo Nordisk A/S, 2880 Bagsvaerd, Denmark

- Present address: Thessa Therapeutics, 038988 Singapore 


\section{ABSTRACT}

Blockade of epidermal growth factor receptor (EGFR) causes tumor regressions in selected patients with metastatic colorectal cancer $(\mathrm{mCRC})$; however, residual disease reservoirs typically remain even after maximal response to therapy, leading to relapse. Using patient-derived xenografts, we observed that $\mathrm{mCRC}$ cells surviving EGFR inhibition exhibited gene expression patterns reminiscent of those displayed by a quiescent subpopulation of normal intestinal secretory precursors with Paneth-cell characteristics. These pseudodifferentiated remnants had reduced expression of EGFR-activating ligands, more pronounced activity of human epidermal growth factor 2 (HER2) and human epidermal growth factor receptor 3 (HER3), and persistent signaling along the phosphatidylinositol-3-kinase (PI3K) pathway compared with untreated tumors. Clinically, residual disease traits were detected in lingering tumors of responsive patients and in tumors of individuals who had experienced early recurrence. Mechanistically, residual tumor reprogramming was mediated by inactivation of Yesassociated protein (YAP) - a master regulator of post-injury intestinal epithelium recovery - following EGFR neutralization. In preclinical trials, Pan-HER antibodies minimized residual disease, blunted PI3K signaling, and induced long-term tumor control after treatment discontinuation. By showing that tolerance to EGFR inhibition is typified by the disengagement of an in-built lineage program that drives both regenerative signaling during intestinal repair and EGFR-dependent tumorigenesis, our results shed light onto $\mathrm{CRC}$ lineage plasticity as an adaptive escape mechanism from therapeutic insults and suggest opportunities to pre-emptively target residual disease.

Single sentence summary: Drug tolerance in EGFR-inhibited mCRC tumors involves Paneth cell-like pseudodifferentiation with rewired, clinically actionable signal dependencies. 


\section{INTRODUCTION}

Although many advanced-stage solid cancers regress when treated with therapies that target drivers of tumor growth, responses are usually incomplete, owing to the presence of residual cells that withstand initial treatment (1). Clinical studies have documented a correlation between depth of response to anticancer therapies and progression-free survival (PFS) (2-4), supporting the notion that the pool of cancer cells that persist in the face of therapy can ultimately be responsible for therapyresistant tumor progression.

The mechanisms that sustain residual tumor burden remain unclear. Cell line-based experiments have shown that exposure to an initial onslaught of potentially lethal growth-inhibitory cues (such as anti-cancer agents) prompts the emergence of a drug-tolerant state, in which drug-resistant subpopulations arise de novo from a drug-sensitive population $(5,6)$. These 'drug-tolerant persisters' (5) occasionally divide and fail to undergo cell death under drug pressure; moreover, they resume vigorous proliferation when therapy is withdrawn and regain drug sensitivity after a washout period, indicating a reversible phenotype that is stochastically attained and relinquished starting from a bulk of heterogeneous cells. The pool of drug-tolerant cancer cells that survive initial therapy can evolve subclones with more permanent, genetically determined mechanisms of acquired resistance. For example, after prolonged drug exposure, EGFR-mutant non-small cell lung cancer (NSCLC) cells that tolerate EGFR blockade give rise to newly emerging subpopulations harboring a gatekeeper resistance mutation $(6,7)$.

In vitro studies have crucially contributed to conceptualizing the basic principles of drug tolerance to targeted therapies. However, more clinically relevant attempts to understand how these principles apply to residual disease in human tumors have been scarce so far (8-10). This is due to inherent hurdles when using tumor material from patients, including the difficulty in collecting and analyzing residual cells at maximal tumor response, the paucity of clinical specimens from treated patients, and a dearth of experimental models that accurately recapitulate fundamental aspects of residual disease and recurrence (1). We decided to address this knowledge gap with a focus on metastatic colorectal cancer $(\mathrm{mCRC})$, a tumor setting for which the clinical challenge of residual disease is particularly germane. Monoclonal antibodies that inhibit EGFR (cetuximab and panitumumab) achieve clinically meaningful rates of response in chemorefractory patients with inoperable mCRC tumors (11-15). Several laboratories, including ours, have identified a number of genetic alterations that are associated with and causally responsible for innate resistance to EGFR blockade in mCRC (16-20). Although omitting anti-EGFR therapy in mCRC patients carrying these resistance-associated genotypes has refined the identification of sensitive cases, complete regressions remain exceptions. This translates into a suboptimal outlook for responsive patients, with only 2-month improvement in progression-free survival (PFS) and 6-month improvement in overall survival compared with chemotherapy (15). This 
evidence highlights the importance of understanding the molecular bases of drug tolerance in EGFRinhibited $\mathrm{mCRC}$ as a prelude to limiting residual disease and delaying the development of resistance.

Here we used mCRC patient-derived xenografts (PDXs), captured at maximal response to cetuximab, to disentangle the phenotypic and functional ramifications of EGFR inhibition in drugtolerant persisters. We report that EGFR blockade in CRC i) disrupts a feed-forward homeostatic circuit that maintains tumor reliance on EGFR signals by co-opting pro-regenerative programs active in the normal intestine after injury, and ii) unleashes pseudodifferentiation towards an EGFR-independent, Paneth cell-like phenotype with alternate therapeutic vulnerabilities. More broadly, this study illustrates the importance of lineage-based adaptive reprogramming as a means to evade pathway dependency and oppose tumor eradication by therapy. 


\section{RESULTS}

\section{Residual colorectal tumors after EGFR blockade are made of slowly cycling cells that resemble EGFR-inhibited normal intestinal cells}

For population-level assessment of residual disease after cetuximab therapy we deployed a series of 279 mCRC PDXs, derived from as many patients, part of which had been used in previous independent studies $(16,20,21)$. Tumor regressions for analysis of residual disease (designated as at least $50 \%$ mean tumor volume reduction compared with pre-treatment volumes) were scored in 40 cases (14.3\%) (Fig. 1A). Consistent with observations in patients (15), all these responsive cases were wild-type for clinically validated resistance mutations (KRAS, NRAS, and BRAF) (Fig. 1A) and for additional alterations that are known to confer resistance or attenuate response (16-21), but have not yet entered routine determination.

Among responsive PDXs, systematic studies for specific biomarkers were conducted on 30 cases (hereafter referred to as 'reference collection') (Fig. 1A). A variable number of these models were used for more complex investigations. In the reference collection, residual disease cells after 6 weeks of therapy exhibited widespread reduction (but not complete abrogation) of cell proliferation compared to the untreated tumors, together with a slight increase in apoptosis (Fig. 1B). Longitudinal evaluation for 12 weeks in 2 cases (CRC0078 and CRC0096) revealed a drastic drop in cell proliferation immediately after therapy initiation, with later resumption of low but continuous mitotic activity beginning 1 (CRC0078) or 3 (CRC0096) weeks after treatment onset (fig. S1A). Cell-cycle slowdown after prolonged treatment with cetuximab was confirmed by reduction of 5-ethynyl-2'-deoxyuridine (EdU) incorporation in both PDXs and organoids (fig. S1B). The number of apoptotic cells fluctuated only marginally over time, without an apparent trend (fig. S1A). The fact that tumor shrinkage was accompanied by reduced cancer cell proliferation in the absence of more pronounced apoptosis can be explained as the result of a new equilibrium imposed by cetuximab-dependent cell-cycle arrest on the spontaneous growth and death dynamics of the tumor mass, with a shift in the net balance between cell production and cell loss that favored basal tumor attrition without increasing the number of apoptotic cells (22). We also monitored response to a long-term stop-and-go schedule of cetuximab in 1 case. Antibody administration in mice with exponentially growing tumors resulted in a rapid (4 weeks) and massive (approximately 80\%) plateau of tumor regression, but failed to achieve tumor eradication (fig. S1C). Therapy was maintained for additional 17 weeks and then suspended. Upon treatment withdrawal, and following a latency of 5 weeks, tumors started regrowing with kinetics and phenotypes similar to treatment-naïve counterparts, and were sensitive to antibody rechallenge (fig. S1C).

To explore whether the residual cells that persist at maximal response originate from genetic selection or stochastic plasticity, we reasoned that if cetuximab favored the outgrowth of genetically defined pre-existing subclones, a population bottleneck would arise with an enrichment of high- 
frequency somatic mutations (fig. S2A); conversely, a lack of substantial modifications in the frequency distribution of sequence variants would be compatible with functional plasticity (fig. S2B). Wholeexome sequencing (WES) in 2 models of the reference collection did not reveal an enrichment for somatic mutations either in cetuximab-treated samples or in tumors that had relapsed following drug withdrawal, when compared to untreated tumors (fig. S2, C and D). Since our survey was restricted to protein-coding genes, we cannot formally exclude therapy-induced changes in the mutational profile of DNA regulatory regions. However, our results suggest that cetuximab pressure does not cause a measurable expansion of subclonal populations; rather, residual cancer cells likely undergo plastic phenotypic changes in the absence of substantial genetic selection. To achieve higher resolution, we also investigated whether rare subclones harboring known cetuximab resistance mutations had emerged during prolonged antibody treatment in 5 PDX models. Sequenom genotyping for hotspot mutations in 9 genes and Nanostring evaluation of copy number alterations in 11 genes documented a wild-type euploid or nearly euploid status for established resistance-associated genotypes in residual tumors after therapy (data file S1). A hitherto unrecognized Y1021S mutation in the PIK3CA gene, with no demonstrated connection to cetuximab resistance, was present at a $10 \%$ allele frequency in 1 of 2 post-cetuximab samples obtained from 1 model. Collectively, these results indicate that residual disease in PDXs has the identifying characteristics of drug tolerance, as observed in cultured cell lines (5): smoldering growth and minimal apoptosis under drug pressure; a metastable state of reversible sensitivity to therapy; and no acquisition or selection of resistance-conferring genetic alterations.

To better understand the molecular features of residual disease, we carried out a microarraybased gene expression analysis in 18 cetuximab-sensitive PDX models (including 12 models from the reference collection) and extracted paired information on vehicle- versus cetuximab-treated tumors (GSE108277). Combined gene set enrichment analysis (GSEA) and ingenuity pathway analysis (IPA) confirmed the observed reduction of cell proliferation in treated tumors, with no evident signs of apoptosis, and revealed a metabolic switch from glycolysis to lipid metabolism and oxidative phosphorylation (Fig. 1C and data files S2 and S3). This conforms to findings in pancreatic cancer, in which reliance on mitochondrial respiration and impaired glycolytic function have been described as hallmarks of a subpopulation of quiescent tumor cells that survive extinction of KRAS oncogenic signals and are eventually responsible for tumor relapse (23).

It has been recently demonstrated that normal murine intestinal stem cells undergo a state of reversible quiescence after EGFR inhibition (24), similar to the growth disadvantage observed in cetuximab-treated tumors. Accordingly, the transcriptional rewiring triggered by EGFR blockade in tumors echoed that of EGFR-inhibited normal stem cells (Fig. 1D). Analogies between the two contexts also occurred at the functional level. EGFR-inhibited quiescent stem cells of the normal mouse intestine display more pronounced Wnt signal strength compared to rapidly cycling cells with active EGFR signaling (24). Likewise, the nuclear localization of $\beta$-catenin (Fig. 1E) and the expression of the 
canonical Wnt target genes LGR5, LEF1 and ASCL2 (Fig. 1F), all indicative of Wnt activation, were generally higher in residual tumors than in vehicle-treated controls of the reference collection. Stronger Wnt signaling in drug-tolerant cells was confirmed by ChIP-seq analysis in 2 representative models, in which prolonged cetuximab treatment potentiated $\beta$-catenin binding to DNA targets (Fig. $1 \mathrm{G}$ and fig. S3). Similarly, TCF/LEF reporter assays in colospheres revealed increased $\beta$-catenin-dependent transcriptional activity over time after EGFR inhibition (Fig. 1H). Time-course experiments in the two PDX models treated with cetuximab for 12 weeks documented an increase in the number of cells with higher expression and stronger nuclear staining of $\beta$-catenin starting one (CRC0078) or two (CRC0096) weeks after treatment initiation and persisting until therapy was terminated (fig. S4).

\section{Cancer cells that withstand EGFR inhibition are similar to label-retaining secretory precursors expressing markers of the Paneth-cell lineage}

The gene expression profile induced by EGFR blockade in normal intestinal stem cells shares many traits with that shown basally by slowly cycling, DNA-label-retaining cells (LRCs) (24), a reserve population that accounts for tissue regeneration after damage (25). In keeping with the observation that cetuximab-tolerant cancer cells are reminiscent of rarely dividing cells in the normal tissue, we observed upregulation of 3 typical LRC markers (BMI1, HOPX, LRIG1) (26) in treated tumors of the reference collection (Fig. 2A). TERT, another classical LRC marker (26), was instead downregulated in EGFR-inhibited tumors (Fig. 2A), consistent with the fact that EGFR blockade decreases TERT expression (27).

LRCs have been identified as secretory precursors that are committed to mature into Paneth cells and express some genes of the Paneth-cell lineage (28). Accordingly, analysis of our global transcriptomic data revealed that residual PDXs were enriched for a signature of Mex3a-high/Lgr5high cells connoting a murine subpopulation of slowly dividing intestinal stem cells with elevated Wnt signaling and expression of Paneth cell genes (29) (Fig. 2B). The gene expression profile of residual tumors was also enriched for a signature of proper Paneth cells of the mouse small intestine (30) but it did not show commonalities with Reg4+ deep crypt secretory cells, which serve as Paneth cell equivalents in the murine colon (31) (fig. S5). On this ground, we derived a manually curated gene subset that includes established markers of classical Paneth-cell commitment and differentiation, in particular, transcription factors that specify the secretory fate of intestinal progenitors (ATOH1, GFI1, SOX9, XBP1) and markers of Paneth-cell terminal differentiation (DEFA5, DEFA6, LYZ, SPINK4, $D L L 1, D L L 4)$ ('secretory/Paneth-cell signature') (32). By performing GSEA in our microarray dataset, we found that this signature was upregulated in cetuximab-treated PDXs (Fig. 2C). For 5 genes with strong induction by cetuximab ('core signature') (data file S4), results were validated by RT-qPCR in the reference collection (Fig. 2D). Finally, overexpression of the top-ranked markers defensin-5 (DEFA5) and defensin-6 (DEFA6) in residual tumors was substantiated at the protein level, with an 8- 
fold and a 2-fold increase in the average percentage of protein positivity for DEFA5 and DEFA6, respectively (Fig. 2E). DEFA5 positivity in treated tumors was almost invariably associated with $\beta$ catenin nuclear accumulation (fig. S6), compatible with the gene expression data showing the cooccurrence of high Wnt signaling and Paneth cell-like pseudodifferentiation in residual tumors.

To analyze the temporal dynamics of Paneth cell-like phenotypic rewiring, we used two different observation time frames and treatment schedules. First, we analyzed the expression of the secretory/Paneth cell core signature genes over time in the two models in which cetuximab therapy had been prolonged for 12 weeks. Like $\beta$-catenin (fig. S4), many of the core signature genes were upregulated soon after the first dose of cetuximab and continued to be more expressed than in control tumors during the whole period of antibody administration (fig. S7A). At the protein level, the percentage of DEFA6 positivity increased by three folds after two weeks of treatment and remained higher at subsequent time points (fig. S7B). Second, we assessed the expression of the core signature genes at an initial time point (after 3 days of treatment) in the whole reference collection and after cetuximab suspension (when regrown tumors had reached volumes of around $750 \mathrm{~mm}^{3}$, typically several weeks after therapy interruption) in 5 representative cases. Overall, the core signature gene transcripts were upregulated as early as 3 days after the first antibody dose - although for some transcripts the extent of gene modulation was lower than that detected in long-treated tumors - (fig. S7C) and most of them reverted after antibody withdrawal (fig. S7D). Hence, cetuximab induces reversible changes that are rapidly acquired after therapy onset, consolidate in residual disease under continued drug pressure, and dissipate after therapy discontinuation. Principal component analysis of global RNA-seq data from untreated, cetuximab-treated, and regrown samples in 1 PDX model confirmed that tumors that had relapsed after therapy discontinuation showed a transcriptomic profile indistinguishable from that of their untreated counterparts, but radically different from that of ontreatment samples (fig. S7E). This reinforces the notion that the phenotypic rewiring experienced by residual tumors is driven by cancer cell functional plasticity rather than selection of preexisting resistant cells.

Analysis of the baseline gene expression profiles of our series of therapeutically annotated PDXs ( $n=241$; GSE76402) (33) revealed higher expression of the secretory/Paneth-cell signature genes in cetuximab-resistant tumors than in sensitive cases (Fig. $2 \mathrm{~F}$ ). This result holds also when moving to a clinical setting; using a publicly available gene expression dataset comprising CRC metastases from 80 individuals treated with cetuximab monotherapy (GSE5851) (34), we found that patients bearing tumors with high expression of the signature genes had shorter PFS than patients with tumors showing low signature expression (Fig. 2G). Overall, data in PDXs and in patients suggest that secretory/Paneth-cell like traits may be general hallmarks of tolerance to EGFR blockade not only in cancer cell persisters after adaptation to antibody treatment, but also in tumors that are poorly sensitive $a b$ initio. 


\section{Acquisition of the Paneth cell-like phenotype in residual cells is regulated by inactivation of YAP}

YAP, a downstream transcriptional effector of the Hippo pathway, favors post-injury intestinal regeneration by intensifying EGFR pathway activity and transiently suppressing Wnt signaling and Paneth cell differentiation in murine stem cells (35). The Wnt-high, Paneth cell-like phenotype displayed by drug-tolerant tumors led us to speculate that residual disease could be sustained by reduced activation of YAP in EGFR-inhibited cancer cells. We thus compared the gene expression profiles of residual mCRC tumors after cetuximab treatment with those of mice with YAP conditional deletion in the gut (35). The genes with the greatest extent of transcriptional regulation in YAP-knockout mice proved to be highly correlated with the genes most strongly modulated by cetuximab in PDXs (Fig. 3A). Furthermore, a ubiquitous signature of YAP-inducible target genes (36) was potently downmodulated in cetuximab-treated tumors (Fig. 3B). In accordance with a phenotype of signal deactivation, expression and/or nuclear accumulation of YAP were reduced in most residual PDXs of the reference collection (Fig. 3C). YAP protein reduction, along with transcriptional downregulation of YAP target genes, became visible soon after cetuximab administration and was maintained for several weeks until the end of the experiment (fig. S8, A and B); after therapy suspension, YAP immunoreactivity returned to pre-treatment positivity (fig. $\mathrm{S} 8 \mathrm{C}$ ). We confirmed these results using cetuximab-sensitive CRC cell lines (DiFi and HCA46). In agreement with PDX data, EGFR blockade diminished YAP activity, as demonstrated by reduced YAP-dependent transcriptional activity (Fig. 3D). Cetuximab treatment also slightly increased the phosphorylation of two inhibitory serine residues involved in YAP cytoplasmic retention and degradation (fig. S9A) and reduced the expression of established YAP target genes (fig. S9B). Consistent with the notion that YAP negatively regulates Wnt signaling (33), Wnt target genes were upregulated after RNAi-mediated YAP knockdown (fig. S10) and were downregulated after transduction of a constitutively active form of YAP (YAP-5SA) or - to a lesser extent - after transduction of wild-type YAP (fig. S11).

Among the upstream regulators of YAP activation, the mitogen-activated protein kinase (MAPK) cascade and the PI3K/AKT axis play a prominent role (37-39). Because these signals are typically triggered by EGFR (40), we investigated the contribution of either pathway to cetuximab-induced YAP inhibition. MEK blockade by selumetinib or trametinib was as potent as EGFR inhibition in abating YAP reporter activity (Fig. 3D and fig. S12). However, this effect was not recapitulated by PI3K pathway neutralization by dactolisib (targeting all PI3K isoforms and mTOR), buparlisib (targeting all PI3K isoforms) or alpelisib (targeting PI3K $\alpha$ ) (Fig. 3D and fig. S12). Similarly, the modulation of established YAP target genes was concordant after EGFR or MEK blockade, whereas it was less profound and/or more heterogeneous after PI3K pathway inhibition (fig. S9B). Collectively, these results indicate that cetuximab mainly impinges on the MAPK pathway for YAP inactivation. 
To explore whether cetuximab-dependent YAP inhibition has a role in cancer cell persistence, we simulated a therapeutic setting in established cell-line xenografts. In particular, we analyzed tumor growth kinetics during doxycycline-regulated expression of constitutively active YAP-5SA (fig. S13, A and B) and/or administration of cetuximab, followed by post-treatment monitoring of residual disease burden. After 3 weeks of treatment, DiFi tumors with induced expression of active YAP had grown slower than the non-induced (YAP-5SA-negative) controls (Fig. 3E). Moreover, after treatment withdrawal, tumors from mice previously treated with cetuximab and induced with doxycycline experienced markedly stronger growth disadvantage than tumors from mice treated with cetuximab only (Fig. 3E). Growth retardation in xenografts expressing YAP-5SA suggests that YAP hyperactivation decreases the fitness of EGFR-dependent cells; in drug-tolerant cells surviving prolonged treatment with cetuximab, this effect is exacerbated and translates into weakened restoration of tumor growth following therapy discontinuation. Thus, YAP inhibition is expected to strengthen the viability of EGFR-inhibited cells, instigating cell persistence and contributing to residual disease.

To causally link EGFR blockade, YAP inhibition, and acquisition of the Paneth cell-like phenotype, we modulated EGFR pathway and YAP activity in cell lines and patient-derived models. We confirmed increased expression of the core signature secretory/Paneth cell genes after EGFR inhibition in cell lines and patient-derived cultures, as observed in PDXs (fig. S13C). In analogy with the YAP reporter assays, upregulation of such genes was induced by MEK inhibitors but not by agents targeting the PI3K pathway, both in vitro and in vivo (Fig. 4A and Fig. S13, D and E). This indicates that cetuximabregulated YAP inactivation and induction of the Paneth cell-like phenotype are both primarily mediated by inhibition of the MAPK cascade. Congruent with the assumption that YAP suppresses Paneth cell pseudodifferentiation in CRC cells, secretory/Paneth cell genes were upregulated after RNAi-mediated silencing of YAP (fig. S14A) and some of them were downregulated after exogenous expression of constitutively active YAP-5SA (fig. S14B). Notably, enforced YAP-5SA expression prevented cetuximab-induced upregulation of secretory/Paneth cell markers (Fig. 4B). A reduction in the basal expression of the secretory/Paneth cell genes was also achieved by ectopic overexpression of wildtype YAP (fig. S14B); however, consistent with the fact that wild-type YAP can still be modulated by upstream signals, its ability to blunt the effect of cetuximab on the acquisition of the Paneth cell-like phenotype was weaker than that of YAP-5SA (Fig. 4B). Similar to in vitro data, established DiFi xenografts had increased expression of secretory/Paneth cell genes after cetuximab treatment and decreased expression of the same genes after doxycycline-regulated expression of YAP-5SA (Fig. 4C and fig. S14C); moreover, again in accordance with the in vitro results, conditional YAP-5SA induction in xenografts attenuated cetuximab-dependent upregulation of the secretory/Paneth cell markers, with a particularly pronounced effect for the top-ranked genes DEFA5 and DEFA6 (Fig. 4C). Together, 
these findings support a causal role for YAP inhibition in mediating the lineage rewiring engendered by EGFR blockade, both in vitro and in vivo.

The pro-regenerative program driven by YAP in the normal intestine entails not only suppression of Wnt signaling and Paneth cell differentiation, but also transcriptional induction of EGFR ligands, in particular, amphiregulin (AREG), epiregulin (EREG) and heparin-binding EGF (HBEGF) (35). In line with this evidence, EGFR-inhibited residual PDXs of the reference collection showed slight but diffuse reduction in the quantity of $A R E G, H B E G F$ and $E G F$ and marked downregulation of $E R E G$, together with overexpression of betacellulin (BTC) (fig. S15). High amounts of AREG and EREG correlate with response to EGFR antibodies in $\mathrm{MCRC}$ patients (34), whereas BTC can substitute for HER3 ligands in activating the HER2/HER3 heterodimer $(41,42)$. Therefore, the drug-tolerant state in CRC is characterized by reduced expression of ligands that sustain EGFR dependency and increased expression of a growth factor that can activate alternate survival pathways.

\section{Combined EGFR/PI3K inhibition reduces residual disease in vitro but has little impact on tumor control in vivo}

Analysis of residual tumors after EGFR inhibition revealed widespread reduction of cell proliferation without conspicuous signs of apoptosis (Fig. 1B). Accordingly, cetuximab did not trigger considerable apoptosis (as assessed by caspase-3/7 activation) in DiFi and HCA46 cells (fig. S16A). We reasoned that one way to chart therapeutic vulnerabilities in residual disease was to pinpoint targets that, when co-inhibited together with EGFR, synergistically enhance cell death. EGFR mainly signals via the PI3KAKT, MAPK, JAK-STAT, and NF-KB pathways (40). When used in monotherapy, specific inhibitors against each of these pathways were ineffective (fig. S16A). When combined with cetuximab, the drugs with the strongest pro-apoptotic activity were those selectively intercepting the PI3K-AKT signaling axis (as evidenced by abrogation of AKT phosphorylation after treatment with representative PI3K inhibitors) (Fig. 5A and fig. S16B). Similar to that observed in cell lines, co-inhibition of EGFR and PI3K in CRC0078 colospheres exerted more synergistic effects on reducing cell viability than combined blockade of EGFR and another target (MEK) (fig. S16C); consistently, although concomitant neutralization of EGFR and MEK led to smaller colospheres than cetuximab alone, only co-inhibition of EGFR and PI3K almost completely obliterated the colonies (Fig. 5B). We also evaluated the colospheres for their latency before resuming proliferation after treatment cessation; cells that had survived prolonged treatment with cetuximab alone or cetuximab and the MEK inhibitor selumetinib promptly regained growth ability after drug washout; conversely, concomitant blockade of EGFR and PI3K prevented regrowth after treatment withdrawal (Fig. 5C).

The fact that apoptosis was unleashed only when EGFR and PI3K were concomitantly inhibited suggests that the antitumor effects of cetuximab are attenuated by persistent activation of the PI3K pathway. Indeed, cetuximab treatment almost completely abated extracellular signal-regulated kinase 
(ERK) phosphorylation in cell lines and colospheres, whereas AKT phosphorylation was only partially affected or remained unaltered (fig. S17A). Similarly, in cetuximab-treated PDXs of the reference collection, the phosphorylation of ERK was more markedly reduced than that of ribosomal protein S6 (S6), a downstream effector of PI3K/AKT (fig. S17B); as expected, ERK and S6 phosphorylation was regained in tumors that had resumed growth after cetuximab discontinuation (fig. S17C). We note that a dichotomy between MAPK and PI3K signaling was also observed when delineating the pathways that transduce cetuximab-dependent impairment of YAP activity and upregulation of secretory/Paneth cell genes. In that case, MAPK inhibition better recapitulated the effects of cetuximab compared with PI3K inhibition (Fig. 3D, Fig. 4A, fig. S9B, fig. S12, fig. S13, D and E). Collectively, these results indicate that the outcomes of EGFR inhibition in CRC are largely regulated by inactivation of the MAPK pathway, and are countered by enduring PI3K signaling in residual cells.

Although concomitant inactivation of the EGFR and PI3K pathways increased cancer cell apoptosis in vitro, the burden of residual disease was only partially lessened in mice treated with both EGFR and PI3K inhibitors with respect to mice treated with cetuximab alone. We used the combination of cetuximab and dactolisib versus cetuximab monotherapy in 7 PDX models, all with wild-type forms of the PIK3CA and PTEN genes. In 4 such models, we also tested cetuximab and alpelisib as an alternative PI3K inhibitor. Both compounds were administered at pharmacodynamically active concentrations, as indicated by the specific reduction of phospho-S6 in tumors treated with either inhibitor (fig. S18A) and the decrease of both phospho-S6 and phospho-ERK in tumors treated also with cetuximab (fig. S18B). When dosed as monotherapies, both dactolisib and alpelisib did not induce tumor shrinkage (fig. S18C). The combination of dactolisib and cetuximab proved to be more effective than cetuximab alone in regressing palpable masses in 2/7 (28.6\%) models, and the combination of alpelisib and cetuximab was superior to single-agent cetuximab in 2/4 (50\%) cases (Fig. 5D and fig. S19). In aggregate, response to cetuximab and either dactolisib or alpelisib was better than response to cetuximab alone in $4 / 7$ models (57\%), indicating that the effect of dual EGFR-PI3K blockade was relatively distributed in the PDX subset tested. However, susceptibility to dactolisib or alpelisib was heterogeneous in the models treated with both compounds, likely due to the different targets of the two inhibitors (all PI3K isoforms and mTOR in the case of dactolisib versus PI3K $\alpha$ only for alpelisib) and the different nature of the ensuing signaling feedback loops (43).

Inspection of cancer cell density in PDXs with no macroscopic response revealed decreased representation of epithelial neoplastic islets in $3 / 5(60 \%)$ tumors, which suggests some therapeutic efficacy in a context of preponderant reactive stroma (fig. S20A). Dual inhibition of EGFR and PI3K induced a slight increase in the number of apoptotic cells compared with individual EGFR blockade in some PDX models in which the combination therapy was more effective than cetuximab alone (Fig. 5D and S20B). However, deeper tumor shrinkage was not invariably accompanied by heightened apoptosis; some tumors with stronger induction of apoptosis after combination therapy did not respond 
better in terms of tumor regression, and others with a more pronounced size reduction were not more apoptotic (Fig. 5D and fig. S20B). Similar to our interpretation of cetuximab-induced tumor regression in the absence of overt apoptosis, also in this case tumor shrinkage in models sensitive to the combination therapy was probably caused by the strong antiproliferative effect of dual EGFR-PI3K inhibition (which shifted the steady-state equilibrium between new cell production and cell loss toward tumor breakdown) rather than by apoptosis induction.

The limited extent of residual disease reduction documented above was not sufficient to increase animals' survival; indeed, PI3K blockade on top of cetuximab did not affect time to relapse after treatment discontinuation in the 4 PDX models examined (fig. S20C). In summary, combined EGFR and PI3K blockade was more effective than cetuximab alone in impairing cell viability in vitro; however, this therapy provided suboptimal and model-specific benefits in reducing residual disease at the end of treatment and did not have long-term effects on tumor control after therapy suspension.

\section{Inhibition of the EGFR-YAP axis leads to increased HER2/HER3 signaling in PDXs and in patients}

To identify more effective therapies against residual disease in vivo we searched for compensatory cues triggered by inhibition of the EGFR-YAP axis and expected to sustain persistent PI3K signaling. We focused on HER2 and HER3, prompted by the knowledge that tumor models treated with EGFR or PI3K inhibitors experience feedback activation of such receptors and that HER2/HER3 signaling preferentially impinges on the PI3K pathway (44-47). We started by gauging HER2 and HER3 expression in DiFi and HCA46 cells transduced with either a control vector or various YAP constructs, in the presence or absence of cetuximab. EGFR blockade resulted in increased expression of $H E R 2$ and HER3 in control cells (Fig. 6A); in a complementary fashion, ectopic introduction of constitutively active YAP-5SA lowered the basal amounts of HER2 and/or HER3 (fig. S21A) and blunted their upregulation by cetuximab (Fig. 6A). Likewise, doxycycline-regulated activation of YAP in established DiFi xenografts reduced the extent of HER2 and HER3 induction prompted by cetuximab (Fig. 6B). Similar to that described for YAP-5SA, transduction of wild-type YAP led to a reduction of HER3 basal expression (fig. S21B); however, since wild-type YAP can still be inhibited by upstream signals, its exogenous overexpression was not sufficient to prevent HER2/HER3 upregulation by cetuximab (fig. S21C). In complementary loss-of-function experiments, we found that only the more effective shRNA construct against YAP caused HER3 upregulation while HER2 was downregulated (fig. S21D), possibly because YAP depletion unleashed a compensatory activity by its paralog TAZ. In summary, the surge in HER2/HER3 expression triggered by EGFR inhibition was prevented by hyperactive YAP but it was not phenocopied by YAP silencing, suggesting that YAP is necessary but not sufficient for EGFR-dependent modulation of HER2 and HER3. The observation that HER2 and HER3 can be transcriptionally modulated by YAP activity is in accordance with the presence of binding site peaks 
for TEAD4 (a canonical transcriptional partner of YAP and TAZ) in HER2 and HER3 regulatory regions, as shown by analysis of the ENCODE ChIP-seq datasets (48).

We then analyzed HER2 and HER3 expression in treatment-naïve and cetuximab-treated PDXs of the reference collection. Consistent with results in cultured cell lines and cell-line xenografts, we detected moderate to strong overexpression of HER2 and HER3 in a sizeable fraction of EGFRinhibited models (Fig. 6C). Similar to that reported for Paneth cell genes, HER2/HER3 upregulation was evident as early as one week after the first cetuximab administration and was maintained for 12 weeks under antibody treatment (fig. S22). In situ inspection of tissue sections revealed cytoplasmic expression of DEFA5 in some, but not all, phospho-HER2 and phospho-HER3-positive cells (fig. S23), suggesting that HER2/HER3 activation after cetuximab treatment is regulated by YAP-dependent but also YAP-independent pathways. For example, HER2/HER3 could be activated in "bystander" DEFA5negative cells due to the observed surge in BTC expression after cetuximab treatment (fig. S15).

Of note, the transcriptional rewiring occurring in EGFR-inhibited cell lines and PDXs was also observed in tumors from patients. The present standard of care for KRAS/NRAS-wild-type mCRC is combinatorial treatment with cetuximab/panitumumab and chemotherapy $(14,15)$. Although this regimen is typically administered in the chemorefractory setting, with EGFR antibodies having a preponderant effect over chemotherapy, residual reactions to cytotoxic agents may influence response to EGFR inhibition. To overcome this hurdle, we interrogated samples from patients with $K R A S / N R A S / B R A F$ wild-type tumors who had undergone single-agent therapy with Sym004, a mixture of two recombinant antibodies against EGFR that induces clinically meaningful rates of tumor shrinkage in $\mathrm{mCRC}$ patients with acquired resistance to cetuximab or panitumumab (49). Patients had been monitored in the frame of a controlled clinical trial with planned on-treatment biopsies right before first tumor restaging, with paired tumor tissues including the treatment-naïve surgical specimen after primary tumor resection and the on-treatment biopsy of a metastasis, after 4 weeks of therapy. We detected strong transcriptional induction of HER2 and HER3 in the on-treatment samples of 2 individuals who had experienced a RECIST partial response (-38\%; patient MPP192-5) or a minor tumor shrinkage (-15\%; patient MPP192-1) at the time of metastatic biopsy (Fig. 6D). In patient MPP192-5, who had responded better to Sym004, we also observed transcriptional upregulation of the secretory/Paneth-cell markers DEFA5 and ATOH1 (Fig. 6D) and confirmed DEFA5 overexpression at the protein level (Fig. 6E). Such modulations appeared to be specific to sensitive cases, as the transcripts were not upregulated in samples from 2 patients in whom Sym004 had been poorly active (+6.4\%; patient MPP192-2) or ineffective (+37.8\%; patient MPP192-4) (Fig. 6D).

The notion that EGFR-inhibited tumors experience an adaptive shift from a status of high EGFR pathway activity to a condition of enhanced stimulation of HER2/HER3-dependent signals is strengthened by the observation that residual PDXs not only showed HER2 and HER3 upregulation, but also higher expression of the promiscuous HER ligand BTC (fig. S15). When we modeled this 
context in cell lines, we found that CRC cells exposed to BTC had stronger activation of EGFR, HER2, and HER3, as well as increased phosphorylation of downstream transducers (Fig. 6F). BTC-stimulated cells were also less responsive (or totally refractory, in the case of DiFi) to cetuximab (Fig. 6G), suggesting that BTC counteracts EGFR blockade through compensatory HER2/HER3 activation. To test this hypothesis, we incubated cells with BTC and treated them with Pan-HER, a mixture of 6 antibodies against the 3 HER receptors (50) (for stoichiometric targeting of EGFR, the Pan-HER dosage was adjusted to achieve equimolar concentrations of cetuximab and the anti-EGFR constituent of Pan-HER). Different from cetuximab, Pan-HER was effective in reducing the viability of BTC-treated cells (Fig. 6G), supporting the notion that HER2, HER3 and BTC restrain responsiveness to individual EGFR blockade and impart a new signal dependency to EGFR-inhibited cells. Selective inhibition of HER2 or HER3 with trastuzumab or the isolated anti-HER components of Pan-HER did not affect cell viability either in the presence or in the absence of BTC, indicating that full co-targeting of HER receptors is required to thwart BTC-dependent survival signals (fig. S24). In agreement with increased HER2/HER3 pathway activity in vitro, residual tumors in PDXs of the reference collection displayed increased phosphorylation of HER2 and HER3 (Fig. 6H).

\section{Targeting HER2/HER3 together with EGFR provides long-term therapeutic benefits in PDX trials}

To explore whether compensatory HER2/HER3 signaling contributes to residual disease in vivo, we treated 6 PDX models with cetuximab or Pan-HER. In the 4 PDXs in which cetuximab caused clear regressions, the extent of tumor shrinkage obtained with Pan-HER was similar to that achieved by cetuximab (Fig. 7A). However, resumption of tumor growth after antibody withdrawal was delayed in $3 / 4(75 \%)$ models treated with Pan-HER compared with those treated with cetuximab (Fig. 7B). In 2 models in which cetuximab left a higher burden of residual disease, Pan-HER was much more effective in reducing tumor volumes (Fig. 7C) and, again, in retarding tumor relapse after treatment suspension (Fig. 7D). In total, out of 6 PDX cases tested, mouse cohorts implanted with 5 models (83\%) experienced a survival benefit after therapy cessation.

The prominent activity of Pan-HER was also evident at the signaling level. Consistent with the notion that HER2 and HER3 cooperate for preferential stimulation of the PI3K-AKT pathway, Pan-HER induced stronger inhibition of PI3K-dependent signals than cetuximab - as assessed by S6 phosphorylation - whereas the effects on the MAPK cascade were similar (Fig. 7E and fig. S25). Moreover, Pan-HER impeded the feedback activation of HER2 and HER3 triggered by individual EGFR blockade (Fig. 7E). In summary, Pan-HER minimized residual disease and reduced EGFR downstream signaling in EGFR-dependent mCRC PDXs. This effect translated into tumor control after therapy discontinuation, with longer time to progression than that obtained with cetuximab. 


\section{DISCUSSION}

Following EGFR blockade, actively dividing stem cells of the normal mouse intestine morph into quiescent cells that maintain robust Wnt signaling and share attributes with DNA-label-retaining precursors expressing some secretory markers of the Paneth-cell lineage $(24,28,29)$. Using paired samples of untreated and cetuximab-treated PDXs, here we show that EGFR inhibition in mCRC tumors results in the emergence of residual cancer cell subpopulations that display hyperactive Wnt signaling and express traits of secretory commitment and Paneth cell-like differentiation. Hence, a functional reprogramming that drives quiescence of normal intestinal cells in the mouse is resurrected during acquisition of drug tolerance in human CRC tumors, suggesting a higher-order lineage plasticity that goes beyond species and tissue functional state.

We provide evidence that the Paneth cell-like phenotypic rewiring prompted by EGFR inhibition in colorectal tumors is an effect of YAP inactivation. During intestinal regeneration after injury, YAP reprograms intestinal stem cells by transiently suppressing Paneth cell differentiation, while bolstering EGFR-dependent proliferation mediated by increased production of EGFR ligands (35). In a reciprocal scenario, we found that residual tumors after EGFR inhibition had reduced expression and nuclear localization of YAP, increased expression of Paneth-cell markers, and downregulation of EGFRactivating ligands. These features are likely to be causally linked, as exogenous introduction of a hyperactive form of YAP prevented the induction of the Paneth cell-like phenotype by cetuximab in CRC cell lines. As a further piece of information, we demonstrate here that EGFR activation is not only a consequence of YAP function, but also an upstream regulatory event. This module speaks for a bistable, feed-forward autoregulatory loop that potentiates EGFR activation when the kinase is not constrained, but precipitates EGFR neutralization and fosters evasion from EGFR dependence as soon as the receptor activity is restricted, for example by low ligand availability or by pharmacological inhibition.

Many cetuximab-treated tumors also featured HER2 and HER3 overexpression, which, similar to manifestation of the Paneth cell-like phenotype, can be ascribed to cetuximab-induced inhibition of YAP. YAP inactivation appears therefore to orchestrate both a lineage shift toward the secretory/Paneth cell-like fate and a signaling shift from high EGFR activity to high HER2/HER3 activity. We assume that EGFR pathway rewiring sustains residual disease, as shown by the fact that cumulative targeting of EGFR, HER2 and HER3 induced more pronounced tumor regressions and delayed tumor relapse after treatment cessation in several PDX models; in this context, we interpret the materialization of Paneth-cell-like characteristics as the accompanying phenotype of the change in signal dependency, which likely reflects the conserved memory of an in-built program that controls differentiation-associated quiescence in the normal intestine and residual disease in tumors. We detected HER2/HER3 phosphorylation/activation also in non-Paneth-like (DEFA5-negative) residual 
cells; this suggests that EGFR family adaptive reprogramming after EGFR inhibition may be enacted through additional cooperative mechanisms, for example through BTC upregulation or YAPindependent, MYC-dependent modulation of HER3 expression (51). HER2/HER3 activation, besides driving tolerance in cetuximab-sensitive tumors as a consequence of compensatory gene overexpression, may also sustain upfront antibody resistance on a genetic basis, as shown for mCRC subsets with ERBB2 gene amplification or mutations $(16,19,20)$. Finally, residual disease may be further fostered by implementation of a cancer stem cell program, as we have shown that both EGFR inhibition and YAP inactivation led to increased expression of Wnt target genes known to control stem cell maintenance in the normal intestine and colorectal cancer (25).

When translated to the clinic, our results advocate agents targeting functional dependencies in residual disease be given as upfront therapies or as sequential therapies immediately after maximal response. Such treatments may complement other preemptive strategies aimed to neutralize genetic mechanisms of resistance before clinical manifestation of disease progression, for example by combining EGFR antibodies and MEK inhibitors for contrasting the outgrowth of RAS-mutant subclones (52). Approaches of this kind would be a paradigm shift in clinical decision making by repositioning rational therapeutic intervention from the refractory state, when multiple and preponderant genetic mechanisms of resistance reduce the chances of clinical benefit, to the phase of massive regression.

We are aware that our findings have certain limitations. For example, we have not tested whether Paneth cell-like cells act as tumor-(re)initiating cells following cetuximab cessation, mainly because PDXs are hardly amenable to lineage-tracing approaches. Moreover, we acknowledge that the clinical validation of our results is restricted to a small number of patients. The procurement of paired pre- and on-treatment samples to investigate early adaptive response to therapy remains a hurdle in $\mathrm{mCRC}$, not only for ethical and logistical reasons but also owing to the difficulty of selecting patients with a treatment history and a clinical monitoring that allow for unequivocal interpretation of the results. For accurate clinical investigation of residual disease we decided to interrogate samples from mCRC patients who had received a 'clean' treatment (EGFR antibody monotherapy without concomitant chemotherapeutic agents), even though this conservative choice limited the analysis to 4 patients. Albeit circumscribed to a small cohort of individuals, the results confirmed HER2 and HER3 upregulation in the residual tumors of sensitive patients and overexpression of secretory/Paneth-cell markers in 1 good responder. The clinical relevance of our findings is also backed by evidence that patients experiencing earlier relapse during cetuximab therapy had tumors with higher amounts of secretory/Paneth cell markers than individuals with longer PFS, providing clinical support to the notion that this cell fate reprogramming typifies lingering cells that tend to endure EGFR inhibition.

Transdifferentiation has been associated with drug resistance in NSCLC, prostate cancer, and melanoma $(9,53,54)$. By extending the generality of this mechanism to other tumor types, our results 
illustrate how perturbations of conserved developmental pathways that control cellular plasticity may represent key factors for shaping drug response in cancer, and motivate the design of therapeutic strategies to target the reversible phase of drug tolerance preceding the onset of mutational resistance. 


\section{MATERIALS AND METHODS}

\section{Study design}

This study was designed to identify mechanisms that sustain drug tolerance in residual CRC tumors at maximal response to the EGFR antibody cetuximab and find therapies that, when combined with EGFR blockade, may minimize residual disease and delay tumor regrowth after treatment discontinuation. In the first part of the study, cohorts of nonobese diabetic-severe combined immunodeficient (NOD-SCID) mice bearing tumors expanded from 279 independent PDXs (12 or 24 mice for each original sample) were treated with cetuximab monotherapy to identify cases with objective but incomplete response. In the second part, molecular profiles (gene expression data obtained from oligonucleotide microarrays and/or RT-qPCR) were analyzed to extract the identifying features of residual disease and put forward hypotheses about potential regulatory mechanisms. In the third part, mechanistic studies based on the working hypotheses emerged from the second part were conducted using forward and reverse genetics in cell lines. Finally, candidate targets likely to sustain residual disease were inhibited with specific therapies, alone or in combination with cetuximab, in PDXs. Tumor-bearing mice were randomized to treatment cohorts. Cohort sample sizes were calculated to have $80 \%$ power to detect a twofold change in tumor size between groups, using a twogroup $t$ test with a two-sided type I error rate of 0.05 . End points for animal experiments were selected in accordance with institutional-approved criteria and guidelines from the Italian National Institute of Health. Information on immunohistochemistry and morphometric quantitations, molecular and bioinformatics analyses, and cell-based experimentation can be found in the Supplementary Materials. Details regarding sample number and replication in assays, as well as statistical analysis, are given in figure legends and in the Methods below. All values for quantitation of immunohistochemistry images and tumor growth curves in animal experiments were recorded blindly.

\section{Specimen collection and annotation}

Tumor and matched normal samples were obtained from patients treated by liver metastasectomy at the Candiolo Cancer Institute (Candiolo, Torino, Italy), Mauriziano Umberto I (Torino), and San Giovanni Battista (Torino). All patients provided informed consent. Samples were procured and the study was conducted under the approval of the Review Boards of the Institutions.

\section{PDX models and in vivo treatments}

Tumor implantation and expansion were performed in 6-week-old male and female NOD/SCID mice as previously described (16). Once tumors reached an average volume of $\sim 400 \mathrm{~mm}^{3}$, mice were randomized into treatment arms, with at least $n=5$ per group, and were treated with the modalities indicated in the figures. Dactolisib (Carbosynth) was dissolved in 1 volume of $\mathrm{N}$-methyl-2-pyrrolidone 
(Sigma Aldrich) and 9 volumes of PEG300 (Fluka). Alpelisib (Carbosynth) was dissolved in $0.5 \%$ carboxymethylcellulose (Fluka). Doxycycline was dissolved in water. Tumor size was evaluated onceweekly by caliper measurements, and the approximate volume of the mass was calculated using the formula $4 / 3 \pi \cdot(d / 2)^{2} \cdot D / 2$, where $d$ and $D$ are the minor tumor axis and the major tumor axis, respectively. Results were considered interpretable when a minimum of 4 mice per treatment group reached the pre-specified endpoints (at least 3 weeks on therapy or development of tumors with average volumes larger than $1500 \mathrm{~mm}^{3}$ within each treatment group in trials aimed to assess drug efficacy; at least 3 weeks after treatment cessation or development of individual tumors with volumes larger than $750 \mathrm{~mm}^{3}$ in survival experiments aimed to assess tumor control by therapy). Operators were blinded during measurements. In vivo procedures and related biobanking data were managed using the Laboratory Assistant Suite (55). Animal procedures were approved by the Italian Ministry of Health (authorization 806/2016-PR).

\section{Statistics}

Error bars indicate standard deviations (SD) unless otherwise indicated. The number of biological (nontechnical) replicates for each experiment is reported in the figure legends. For experiments with two groups, statistical analysis was performed using two-tailed Student's $t$-test, Welch's $t$-test or Wilcoxon matched pairs signed-rank test unless otherwise noted. For experiments with more than two groups, one-way ANOVA was used. Two-way ANOVA was applied for experiments in which the determinations were considered interdependent. In case of multiple testing, we adopted the Benjamini, Krieger and Yekutieli false discovery rate (FDR) two-stage step-up method or the Benjamini-Hochberg FDR test. Correlations were calculated by Pearson's coefficients. Statistical analyses in the survival experiments were performed by Log-rank (Mantel-Cox) test. The level of statistical significance was set at $P<0.05$. For multiple comparisons, the results were considered significant when the Benjamini, Krieger and Yekutieli method was $<0.05$ and when the Benjamini-Hochberg FDR was $<0.1$. Graphs were generated and statistical analyses performed using the GraphPad Prism (v8.0) statistical package. Original data are provided in data file S5. 


\section{SUPPLEMENTARY MATERIALS}

Materials and Methods

Figure S1. Biological characterization of residual disease after prolonged treatment with cetuximab in representative mCRC PDXs

Figure S2. Analysis of adaptive changes versus clonal selection induced by cetuximab in representative $\mathrm{mCRC}$ PDXs

Figure S3. ChIP-seq analysis of residual disease after prolonged treatment with cetuximab in representative mCRC PDXs

Figure S4. Longitudinal analysis of $\beta$-catenin expression at different time points during prolonged treatment with cetuximab in representative $\mathrm{mCRC}$ PDXs

Figure S5. GSEA of residual PDXs with signatures of Paneth cells and deep secretory cells

Figure S6. DEFA5 and $\beta$-catenin double staining in representative $\mathrm{mCRC}$ PDXs treated with cetuximab

Figure S7. Transcript and protein changes of Paneth-cell markers and global gene expression variations in representative $\mathrm{mCRC}$ PDXs during different time points of cetuximab treatment and after therapy suspension

Figure S8. Expression of YAP and YAP targets in representative mCRC PDXs during different time points of cetuximab treatment and after therapy suspension

Figure S9. Inhibition of YAP activity and expression of YAP-dependent genes by cetuximab in CRC cell lines

Figure S10. Expression of secretory/Paneth cell genes after YAP silencing in CRC cell lines

Figure S11. YAP-dependent regulation of Wnt target genes in CRC cell lines

Figure S11. Expression of secretory/Paneth cell genes after YAP silencing in CRC cell lines. 
Figure S12. Modulation of YAP transcriptional activity by cetuximab and other inhibitors of the EGFR pathway in CRC cell lines

Figure S13. Expression/activity of doxycycline-inducible YAP-5SA and modulation of secretory/Paneth cell genes by EGFR pathway inhibition in vitro and in vivo

Figure S14. Expression of secretory/Paneth cell genes after YAP silencing or YAP overexpression in vitro and in vivo

Figure S15. Modulation of EGFR family ligands in mCRC PDXs treated with cetuximab

Figure S16. Effects of individual signal inhibition and dual blockade of EGFR and PI3K or EGFR and MEK in CRC cell cultures

Figure S17. Effects of cetuximab on downstream signals in vitro and in vivo

Figure S18. Effects of PI3K inhibitors on downstream signals and tumor growth in vivo

Figure S19. Effects of PI3K inhibition and combined EGFR and PI3K inhibition on mCRC PDX macroscopic residual disease (pre-treatment and end-of-treatment tumor volumes)

Figure S20. Effects of combined EGFR and PI3K inhibition on mCRC PDX microscopic residual disease, apoptosis, and survival

Figure S21. YAP-dependent transcriptional modulation of HER2 and HER3 in CRC cell lines

Figure S22. Modulation of HER2 and HER3 expression in mCRC PDXs during prolonged treatment with cetuximab

Figure S23. DEFA5 and HER2/HER3 double staining in representative mCRC PDXs treated with cetuximab

Figure S24. CRC cell line sensitivity to individual targeting of HER family members

Figure S25. Effects of cetuximab and Pan-HER on EGFR downstream targets in vivo 
Data file S1 (Microsoft Excel format). List of genes subject to mutational and gene copy number analysis

Data file S2 (Microsoft Excel format). GSEA of gene expression changes induced by cetuximab in mCRC PDXs

Data file S3 (Microsoft Excel format). Ingenuity pathway analysis of gene expression changes induced by cetuximab in $\mathrm{mCRC}$ PDXs

Data file S4 (Microsoft Excel format). Expression changes of secretory/Paneth cell genes induced by cetuximab in the reference collection (GSE108277)

Data file S5 (Microsoft Excel format). Original data

Data file S6 (Microsoft Excel format). Taqman probes used for RT-qPCR 


\section{REFERENCES}

1. T.G. Bivona, R.C. Doebele, A framework for understanding and targeting residual disease in oncogene-driven solid cancers. Nat. Med. 22, 472-478 (2016).

2. G. M. Blumenthal, S. W. Karuri, H. Zhang, L. Zhang, S. Khozin, D. Kazandjian, S. Tang, R. Sridhara, P. Keegan, R. Pazdur, Overall response rate, progression-free survival, and overall survival with targeted and standard therapies in advanced non-small-cell lung cancer: US Food and Drug Administration trial-level and patient-level analyses. J. Clin. Oncol. 33, 1008-1014 (2015).

3. H. Piessevaux, M. Buyse, W. De Roock, H. Prenen, M. Schlichting, E. Van Cutsem, S. Tejpar, Radiological tumor size decrease at week 6 is a potent predictor of outcome in chemorefractory metastatic colorectal cancer treated with cetuximab (BOND trial). Ann. Oncol. 20, 1375-1382 (2009).

4. H. Piessevaux, M. Buyse, M. Schlichting, E. Van Cutsem, C. Bokemeyer, S. Heeger, S. Tejpar, Use of early tumor shrinkage to predict long-term outcome in metastatic colorectal cancer treated with cetuximab. J. Clin. Oncol. 31, 3764-3775 (2013).

5. S. V. Sharma, D. Y. Lee, B. Li, M. P. Quinlan, F. Takahashi, S. Maheswaran, U. McDermott, N. Azizian, L. Zou, M. A. Fischbach, K. K. Wong, K. Brandstetter B. Wittner, S. Ramaswamy, M. Classon, J. Settleman, A chromatin-mediated reversible drug-tolerant state in cancer cell subpopulations. Cell 141, 69-80 (2010).

6. A. N. Hata, M. J. Niederst, H. L. Archibald, M. Gomez-Caraballo, F. M. Siddiqui, H. E. Mulvey, Y. E. Maruvka, F. Ji, H. E. Bhang, V. Krishnamurthy Radhakrishna, G. Siravegna, H. Hu, S. Raoof, E. Lockerman, A. Kalsy, D. Lee, C. L. Keating, D. A. Ruddy, L. J. Damon, A. S. Crystal, C. Costa,, Z. Piotrowska, A. Bardelli, A. J. lafrate, R. I. Sadreyev, F. Stegmeier, G. Getz, L. V. Sequist, A. C. Faber, J. A. Engelman, Tumor cells can follow distinct evolutionary paths to become resistant to epidermal growth factor receptor inhibition. Nat. Med. 22, 262-269 (2016).

7. M. Ramirez, S. Rajaram, R. J. Steininger, D. Osipchuk, M. A. Roth, L. S. Morinishi, L. Evans, W. Ji, C. H. Hsu, K. Thurley, S. Wei, A. Zhou, P. R. Koduru, B. A. Posner, L. F. Wu, S. J. Altschuler, Diverse drug-resistance mechanisms can emerge from drug-tolerant cancer persister cells. Nat. Commun. 7, 10690 (2016).

8. C. Song, M. Piva, L. Sun, A. Hong, G. Moriceau, X. Kong, H. Zhang, S. Lomeli, J. Qian, C. C. Yu, R. Damoiseaux, M. C. Kelley, K. B. Dahlman, P. O. Scumpia, J. A. Sosman, D. B. Johnson, A. Ribas, W. Hugo, R. S. Lo, Recurrent tumor cell-intrinsic and -extrinsic alterations during 
MAPKi-induced melanoma regression and early adaptation. Cancer Discov. 7, 1248-1265 (2017).

9. F. Rambow, A. Rogiers, O. Marin-Bejar, S. Aibar, J. Femel, M. Dewaele, P. Karras, D. Brown, Y. H. Chang, M. Debiec-Rychter, C. Adriaens, E. Radaelli, P. Wolter, O. Bechter, R. Dummer, M. Levesque, A. Piris, D. T. Frederick, G. Boland, K. T. Flaherty, J. van den Oord, T. Voet, S. Aerts, A. W. Lund, J. C. Marine, Toward Minimal Residual Disease-Directed Therapy in Melanoma. Cell 174, 843-855 (2018).

10. K. N. Shah, R. Bhatt, J. Rotow, J. Rohrberg, V. Olivas, V. E. Wang, G. Hemmati, M. M. Martins, A. Maynard, J. Kuhn, J. Galeas, H. J. Donnella, S. Kaushik, A. Ku, S. Dumont, G. Krings, H. J. Haringsma, L. Robillard, A. D. Simmons, T. C. Harding, F. McCormick, A. Goga, C. M. Blakely, T. G. Bivona, S. Bandyopadhyay, Aurora kinase A drives the evolution of resistance to third-generation EGFR inhibitors in lung cancer. Nat. Med. 25, 111-118 (2019).

11. D. Cunningham, Y. Humblet, S. Siena, D. Khayat, H. Bleiberg, A. Santoro, D. Bets, M. Mueser, A. Harstrick, C. Verslype, I. Chau, E. Van Cutsem, Cetuximab monotherapy and cetuximab plus irinotecan in irinotecan-refractory metastatic colorectal cancer. N. Engl. J. Med. 351, 337345 (2004).

12. E. Van Cutsem, M. Peeters, S. Siena, Y. Humblet, A. Hendlisz, B. Neyns, J. L. Canon, J. L. Van Laethem, J. Maurel, G. Richardson, M. Wolf, R. G. Amado, Open-label phase III trial of panitumumab plus best supportive care compared with best supportive care alone in patients with chemotherapy-refractory metastatic colorectal cancer. J. Clin. Oncol. 25, 1658-1664 (2007).

13. R. G. Amado, M. Wolf, M. Peeters, E. Van Cutsem, S. Siena, D. J. Freeman, T. Juan, R. Sikorski, S. Suggs, R. Radinsky, S. D. Patterson, D. D. Chang, Wild-type KRAS is required for panitumumab efficacy in patients with metastatic colorectal cancer. J. Clin. Oncol. 26, 162634 (2008).

14. C. S. Karapetis, S. Khambata-Ford, D. J. Jonker, C. J. O'Callaghan, D. Tu, N. C. Tebbutt, R. J. Simes, H. Chalchal, J. D. Shapiro, S. Robitaille, T. J. Price, L. Shepherd, H. J. Au, C. Langer, M. J. Moore, J. R. Zalcberg, K-ras mutations and benefit from cetuximab in advanced colorectal cancer. N. Engl. J. Med. 359, 1757-1765 (2008).

15. J. Y. Douillard, K. S. Oliner, S. Siena, J. Tabernero, R. Burkes, M. Barugel, Y. Humblet, G. Bodoky, D. Cunningham, J. Jassem, F. Rivera, I. Kocákova, P. Ruff, M. Błasińska-Morawiec, M. Šmakal, J. L. Canon, M. Rother, R. Williams, A. Rong, J. Wiezorek, R. Sidhu, S. D. 
Patterson, Panitumumab-FOLFOX4 treatment and RAS mutations in colorectal cancer. $N$. Engl. J. Med. 369, 1023-1034 (2013).

16. A. Bertotti, G. Migliardi, F. Galimi, F. Sassi, D. Torti, C. Isella, D. Corà, F. Di Nicolantonio, M. Buscarino, C. Petti, D. Ribero, N. Russolillo, A. Muratore, P. Massucco, A. Pisacane, L. Molinaro, E. Valtorta, A. Sartore-Bianchi, M. Risio, L. Capussotti, M. Gambacorta, S. Siena, E. Medico, A. Sapino, S. Marsoni, P. M. Comoglio, A. Bardelli, L. Trusolino, A molecularly annotated platform of patient-derived xenografts ("xenopatients") identifies HER2 as an effective therapeutic target in cetuximab-resistant colorectal cancer. Cancer Discov. 1, 508$523(2011)$.

17. K. Yonesaka, K. Zejnullahu, I. Okamoto, T. Satoh, F. Cappuzzo, J. Souglakos, D. Ercan, A. Rogers, M. Roncalli, M. Takeda, Y. Fujisaka, J. Philips, T. Shimizu, O. Maenishi, Y. Cho, J. Sun, A. Destro, K. Taira, K. Takeda, T. Okabe, J. Swanson, H. Itoh, M. Takada, E. Lifshits, K. Okuno, J.A. Engelman, R.A. Shivdasani, K. Nishio, M. Fukuoka, M. Varella-Garcia, K. Nakagawa, P.A. Jänne, Activation of ERBB2 signaling causes resistance to the EGFRdirected therapeutic antibody cetuximab. Sci. Transl. Med. 3, 99ra86 (2011).

18. A. Bardelli, S. Corso, A. Bertotti, S. Hobor, E. Valtorta, G. Siravegna, A. Sartore-Bianchi, E. Scala, A. Cassingena, D. Zecchin, M. Apicella, G. Migliardi, F. Galimi, C. Lauricella, C. Zanon, T. Perera, S. Veronese, G. Corti, A. Amatu, M. Gambacorta, L.A. Diaz Jr, M. Sausen, V.E. Velculescu, P. Comoglio, L. Trusolino, F. Di Nicolantonio, S. Giordano, S. Siena, Amplification of the MET receptor drives resistance to anti-EGFR therapies in colorectal cancer. Cancer Discov. 3, 658-673 (2013).

19. S. M. Kavuri, N. Jain, F. Galimi, F. Cottino, S. M. Leto, G. Migliardi, A. C. Searleman, W Shen, J. Monsey, L. Trusolino, S. A. Jacobs, A. Bertotti, R. Bose, HER2 activating mutations are targets for colorectal cancer treatment. Cancer Discov. 5, 832-541 (2015).

20. A. Bertotti, E. Papp, S. Jones, V. Adleff, V. Anagnostou, B. Lupo, M. Sausen, J. Phallen, C. A. Hruban, C. Tokheim, N. Niknafs, M. Nesselbush, K. Lytle, F. Sassi, F. Cottino, G. Migliardi, E. R. Zanella, D. Ribero, N. Russolillo, A. Mellano, A. Muratore, G. Paraluppi, M. Salizzoni, S. Marsoni, M. Kragh, J. Lantto, A. Cassingena, Q. K. Li, R. Karchin, R. Scharpf, A. SartoreBianchi, S. Siena, L. A. Diaz Jr., L. Trusolino, V. E. Velculescu, The genomic landscape of response to EGFR blockade in colorectal cancer. Nature 526, 263-267 (2015).

21. E. R. Zanella, F. Galimi, F. Sassi, G. Migliardi, F. Cottino, S. M. Leto, B. Lupo, J. Erriquez, C. Isella, P. M. Comoglio, E. Medico, S. Tejpar, E. Budinská, L. Trusolino, A. Bertotti, IGF2 is an actionable target that identifies a distinct subpopulation of colorectal cancer patients with marginal response to anti-EGFR therapies. Sci. Transl. Med. 7, 272ra12 (2015). 
22. I. A. Rodriguez-Brenes, N.L. Komarova, D. Wodarz, Tumor growth dynamics: insights into evolutionary processes. Trends Ecol. Evol. 28, 597-604 (2013).

23. A. Viale, P. Pettazzoni, C. A. Lyssiotis, H. Ying, N. Sánchez, M. Marchesini, A. Carugo, T. Green, S. Seth, V. Giuliani, M. Kost-Alimova, F. Muller, S. Colla, L. Nezi, G. Genovese, A. K. Deem, A. Kapoor, W. Yao, E. Brunetto, Y. Kang, M. Yuan, J. M. Asara, Y. A. Wang, T. P. Heffernan, A. C. Kimmelman, H. Wang, J. B. Fleming, L. C. Cantley, R. A. DePinho, G. F. Draetta, Oncogene ablation-resistant pancreatic cancer cells depend on mitochondrial function. Nature 514, 628-32 (2014).

24. O. Basak, J. Beumer, K. Wiebrands, H. Seno, A. van Oudenaarden, H. Clevers, Induced quiescence of Lgr5+ stem cells in intestinal organoids enables differentiation of hormoneproducing enteroendocrine cells. Cell Stem Cell 20, 177-190 (2017).

25. H. Clevers, Stem cells: a unifying theory for the crypt. Nature 495, 53-54 (2013).

26. N. Barker, A. van Oudenaarden, H. Clevers, Identifying the stem cell of the intestinal crypt: strategies and pitfalls. Cell Stem Cell 11, $452-460$ (2012).

27. A. Budiyanto, T. Bito, M. Kunisada, M. Ashida, M. Ichihashi, M. Ueda, Inhibition of the epidermal growth factor receptor suppresses telomerase activity in HSC-1 human cutaneous squamous cell carcinoma cells. J. Invest. Dermatol. 121, 1088-1094 (2003).

28. S. J. Buczacki, H. I. Zecchini, A. M. Nicholson, R. Russell, L. Vermeulen, R. Kemp, D. J. Winton, Intestinal label-retaining cells are secretory precursors expressing Lgr5. Nature 495, 65-69 (2013).

29. F. M. Barriga, E. Montagni, M. Mana, M. Mendez-Lago, X. Hernando-Momblona, M. Sevillano, A. Guillaumet-Adkins, G. Rodriguez-Esteban, S. J. A. Buczacki, M. Gut, H. Heyn, D. J. Winton, O. H. Yilmaz, C. S. Attolini, I. Gut, E. Batlle, Mex3a Marks a Slowly Dividing Subpopulation of Lgr5+ Intestinal Stem Cells. Cell Stem Cell 20, 801-816 (2017).

30. A. L. Haber, M. Biton, N. Rogel, R. H. Herbst, K. Shekhar, C. Smillie, G. Burgin, T. M. Delorey, M. R. Howitt, Y. Katz, I. Tirosh, S. Beyaz, D. Dionne, M. Zhang, R. Raychowdhury, W. S. Garrett, O. Rozenblatt-Rosen, H. N. Shi, O. Yilmaz, R. J. Xavier, A. Regev, A single-cell survey of the small intestinal epithelium. Nature 551, 333-339 (2017).

31. N. Sasaki, N. Sachs, K. Wiebrands, S. I. Ellenbroek, A. Fumagalli, A. Lyubimova, H. Begthel, M. van den Born, J. H. van Es, W. R. Karthaus, V. S. Li, C. López-Iglesias, P. J. Peters, J. van Rheenen, A. van Oudenaarden, $H$. Clevers, Reg4+ deep crypt secretory cells function as epithelial niche for Lgr5+ stem cells in colon. Proc. Natl. Acad. Sci. USA 113, E5399-5407 (2016). 
32. H. C. Clevers, C. L. Bevins, Paneth cells: maestros of the small intestinal crypts. Annu. Rev. Physiol. 75, 289-311 (2013).

33. C. Isella, F. Brundu, S. E. Bellomo, F. Galimi, E. Zanella, R. Porporato, C. Petti, A. Fiori, F. Orzan, R. Senetta, C. Boccaccio, E. Ficarra, L. Marchionni, L. Trusolino, E. Medico, A. Bertotti, Selective analysis of cancer-cell intrinsic transcriptional traits defines novel clinically relevant subtypes of colorectal cancer. Nat. Commun. 8, 15107 (2017).

34. S. Khambata-Ford, C.R. Garrett, N.J. Meropol, M. Basik, C.T. Harbison, S. Wu, T.W. Wong, X. Huang, C.H. Takimoto, A.K. Godwin, B.R. Tan, S.S. Krishnamurthi, H.A. Burris 3rd, E.A. Poplin, M. Hidalgo, J. Baselga, E.A. Clark, D.J. Mauro, Expression of epiregulin and amphiregulin and $\mathrm{K}$-ras mutation status predict disease control in metastatic colorectal cancer patients treated with cetuximab. J. Clin. Oncol. 25, 3230-3237 (2007).

35. A. Gregorieff, Y. Liu, M. R. Inanlou, Y. Khomchuk, J. L. Wrana, Yap-dependent reprogramming of Lgr5(+) stem cells drives intestinal regeneration and cancer. Nature 526, 715-718 (2015).

36. S. Dupont, L. Morsut, M. Aragona, E. Enzo, S. Giulitti, M. Cordenonsi, F. Zanconato, J. Le Digabel, M. Forcato, S. Bicciato, N. Elvassore, S. Piccolo, Role of YAP/TAZ in mechanotransduction. Nature 474, 179-183 (2011).

37. B. V. Reddy, K. D. Irvine, Regulation of Hippo signaling by EGFR-MAPK signaling through Ajuba family proteins. Dev. Cell 24, 459-471 (2013).

38. L. Lin, A. J. Sabnis, E. Chan, V. Olivas, L. Cade, E. Pazarentzos, S. Asthana, D. Neel, J. J. Yan, X. Lu, L. Pham, M. M. Wang, N. Karachaliou, M. G. Cao, J. L. Manzano, J. L. Ramirez, J. M. Torres, F. Buttitta, C. M. Rudin, E. A. Collisson, A. Algazi, E. Robinson, I. Osman, E. Muñoz-Couselo , J. Cortes , D. T. Frederick, Z. A. Cooper, M. McMahon, A. Marchetti, R. Rosell, K. T. Flaherty, J. A. Wargo, T. G. Bivona, The Hippo effector YAP promotes resistance to RAF- and MEK-targeted cancer therapies. Nat. Genet. 47, 250-256 (2015).

39. S. Basu, N. F. Totty, M. S. Irwin, M. Sudol, J. Downward, Akt phosphorylates the Yesassociated protein, YAP, to induce interaction with 14-3-3 and attenuation of p73-mediated apoptosis. Mol. Cell 11, 11-23 (2003).

40. Y. Yarden Y, G. Pines. The ERBB network: at last, cancer therapy meets systems biology. Nat. Rev. Cancer 12, 553-563 (2012).

41. R. Pinkas-Kramarski, A. E. Lenferink, S. S. Bacus, L. Lyass, M. L. van de Poll, L. N. Klapper, E. Tzahar, M. Sela, E. J. van Zoelen, Y. Yarden Y, The oncogenic ErbB-2/ErbB-3 heterodimer is a surrogate receptor of the epidermal growth factor and betacellulin. Oncogene 16, 12491258 (1998). 
42. M. Alimandi, L. M. Wang, D. Bottaro, C. C. Lee, A. Kuo, M. Frankel, P. Fedi, C. Tang, M. Lippman, J. H. Pierce, Epidermal growth factor and betacellulin mediate signal transduction through co-expressed ErbB2 and ErbB3 receptors. EMBO J. 16, 5609-5617 (1997).

43. A. Sathe, G. Chalaud, I. Oppolzer, K. Y. Wong, M. von Busch, S. C. Schmid, Z. Tong, M. Retz, J. E. Gschwend, W. A. Schulz, R. Nawroth, Parallel PI3K, AKT and mTOR inhibition is required to control feedback loops that limit tumor therapy. PLoS One 13, e0190854 (2018).

44. M. Mancini, N. Gaborit, M. Lindzen, T. M. Salame, M. Dall'Ora, M. Sevilla-Sharon, A. AbdulHai, J. Downward, Y. Yarden, Combining three antibodies nullifies feedback-mediated resistance to erlotinib in lung cancer. Sci. Signal. 8, ra53 (2015).

45. A. Bosch-Vilaró, B. Jacobs, V. Pomella, L. Abbasi Asbagh, R. Kirkland, J. Michel, S. Singh, X. Liu, P. Kim, G. Weitsman, P. R. Barber, B. Vojnovic, T. Ng, S. Tejpar, Feedback activation of HER3 attenuates response to EGFR inhibitors in colon cancer cells. Oncotarget 8, 4277-4288 (2017).

46. S. Chandarlapaty, A. Sawai, M. Scaltriti, V. Rodrik-Outmezguine, O. Grbovic-Huezo, V. Serra, P. K. Majumder, J. Baselga, N. Rosen, AKT inhibition relieves feedback suppression of receptor tyrosine kinase expression and activity. Cancer Cell 19, 58-71 (2011).

47. K. Gala, S. Chandralapaty, Molecular pathways: HER3 targeted therapy. Clin. Cancer Res. 20, 1410-16 (2014).

48. ENCODE Project Consortium, An integrated encyclopedia of DNA elements in the human genome. Nature 489, 57-74 (2012).

49. R. Dienstmann, A. Patnaik, R. Garcia-Carbonero, A. Cervantes, M. Benavent, S. Susana Roselló, B. B. Tops, R. van der Post, G. Argilés, N. J. Skartved, U. H. Hansen, R. Hald, M. W. Pedersen, M. Kragh, I. D. Horak, S. Braun, E. Van Cutsem, A. W. Tolcher, J. Tabernero, Safety and activity of the first-in-class Sym004 anti-EGFR antibody mixture in patients with refractory colorectal cancer. Cancer Discov. 5, 598-609 (2015).

50. H. J. Jacobsen, T. T. Poulsen, A. Dahlman, I. Kjær, K. Koefoed, J. W. Sen, D. Weilguny, B. Bjerregaard, C. R. Andersen, I. D. Horak, M. W. Pedersen, M. Kragh, J. Lantto, Pan-HER, an antibody mixture simultaneously targeting EGFR, HER2, and HER3, effectively overcomes tumor heterogeneity and plasticity. Clin. Cancer Res. 21, 4110-4122 (2015).

51. C. Sun, S. Hobor, A. Bertotti, D. Zecchin, S. Huang, F. Galimi, F. Cottino, A. Prahallad, W. Grernrum, A. Tzani, A. Schlicker, L. F. Wessels, E. F. Smit, E. Thunnissen, P. Halonen, C. Lieftink, R. L. Beijersbergen, F. Di Nicolantonio, A. Bardelli, L. Trusolino, R. Bernards, Intrinsic 
resistance to MEK inhibition in KRAS mutant lung and colon cancer through transcriptional induction of ERBB3. Cell Rep. 7, 86-93 (2014).

52. S. Misale, I. Bozic, J. Tong, A. Peraza-Penton, A. Lallo, F. Baldi, K. H. Lin, M. Truini, L. Trusolino, A. Bertotti, F. Di Nicolantonio, M. A. Nowak, L. Zhang, K. C. Wood, A. Bardelli, Vertical suppression of the EGFR pathway prevents onset of resistance in colorectal cancers. Nat. Commun. 6, 8305 (2015).

53. L. V. Sequist, B. A. Waltman, D. Dias-Santagata, S. Digumarthy, A. B. Turke, P. Fidias, K. Bergethon, A. T. Shaw, S. Gettinger, A. K. Cosper, S. Akhavanfard, R. S. Heist, J. Temel, J. G. Christensen, J. C. Wain, T. J. Lynch, K. Vernovsky, E. J. Mark, M. Lanuti, A. J. lafrate, M. Mino-Kenudson, J. A. Engelman, Genotypic and histological evolution of lung cancers acquiring resistance to EGFR inhibitors. Sci. Transl. Med. 3, 75ra26 (2011).

54. M. Zou, R. Toivanen, A. Mitrofanova, N. Floch, S. Hayati, Y. Sun, C. Le Magnen, D. Chester, E. A. Mostaghel, A. Califano, M. A. Rubin, M. M. Shen, C. Abate-Shen, Transdifferentiation as a mechanism of treatment resistance in a mouse model of castration-resistant prostate cancer. Cancer Discov. 7, 736-749 (2017).

55. E. Baralis, A. Bertotti, A. Fiori, A. Grand, LAS: a software platform to support oncological data management. J. Med. Syst. 36 Suppl. 1, S81-90 (2012).

56. S. Jammula, D. Pasini, EpiMINE, a computational program for mining epigenomic data. Epigenetics Chromatin 9, 42 (2016).

57. P. Luraghi, G. Reato, E. Cipriano, F. Sassi, F. Orzan, V. Bigatto, F. De Bacco, E. Menietti, M. Han, W. M. Rideout 3rd, T. Perera, A. Bertotti, L. Trusolino, P. M. Comoglio, C. Boccaccio, MET signaling in colon cancer stem-like cells blunts the therapeutic response to EGFR inhibitors. Cancer Res. 74, 1857-1869 (2014).

58. E. Pupo, N. Ducano, B. Lupo, E. Vigna, D. Avanzato, T. Perera, L. Trusolino, L. Lanzetti, P. M. Comoglio, Rebound Effects Caused by Withdrawal of MET Kinase Inhibitor Are Quenched by a MET Therapeutic Antibody. Cancer Res. 76, 5019-5029 (2016).

59. F. lorio, T. A. Knijnenburg, D. J. Vis, G. R. Bignell, M. P. Menden, M. Schubert, N. Aben, E. Gonçalves, S. Barthorpe, H. Lightfoot, T. Cokelaer, P. Greninger, E. van Dyk, H. Chang, H. de Silva, H. Heyn, X. Deng, R. K. Egan, Q. Liu, T. Mironenko, X. Mitropoulos, L. Richardson, J. Wang, T. Zhang, S. Moran, S. Sayols, M. Soleimani, D. Tamborero, N. Lopez-Bigas, P. RossMacdonald, M. Esteller, N. S. Gray, D. A. Haber, M. R. Stratton, C. H. Benes, L. F. A. Wessels, J. Saez-Rodriguez, U. McDermott, M. J. Garnett, A landscape of pharmacogenomic interactions in cancer. Cell 166, 740-754 (2016). 
60. G. Hrustanovic, V. Olivas, E. Pazarentzos, A. Tulpule, S. Asthana, C. M. Blakely, R. A. Okimoto, L. Lin, D. S. Neel, S. Sabnis, J. Flanagan, E. Chan, M. Varella-Garcia, D. L. Aisner, A. Vaishnavi, S. H. Ou, E. A. Collisson, E. Ichihara, P. C. Mack, C. M. Lovly, N. Karachaliou, R. Rosell, J. W. Riess, R. C. Doebele, T. G. Bivona, RAS-MAPK dependence underlies a rational polytherapy strategy in EML4-ALK-positive lung cancer. Nat. Med. 21, 1038-1047 (2015).

61. S. M. Leto, F. Sassi, I. Catalano, V. Torri, G. Migliardi, E. R. Zanella, M. Throsby, A. Bertotti, L. Trusolino, Sustained Inhibition of HER3 and EGFR Is Necessary to Induce Regression of HER2-Amplified Gastrointestinal Carcinomas. Clin. Cancer Res. 21, 5519-5531 (2015).

62. F. Di Nicolantonio, S. Arena, J. Tabernero, S. Grosso, F. Molinari, T. Macarulla, M. Russo, C. Cancelliere, D. Zecchin, L. Mazzucchelli, T. Sasazuki, S. Shirasawa, M. Geuna, M. Frattini, J. Baselga, M. Gallicchio, S. Biffo, A. Bardelli, Deregulation of the PI3K and KRAS signaling pathways in human cancer cells determines their response to everolimus. J. Clin. Invest. 120, 2858-2866 (2010).

63. J. Tsai, J. T. Lee, W. Wang, J. Zhang, H. Cho, S. Mamo, R. Bremer, S. Gillette, J. Kong, N. K. Haass, K. Sproesser, L. Li, K. S. Smalley, D. Fong, Y. L. Zhu, A. Marimuthu, H. Nguyen, B. Lam, J. Liu, I. Cheung, J. Rice, Y. Suzuki, C. Luu, C. Settachatgul, R. Shellooe, J. Cantwell, S. H. Kim, J. Schlessinger, K. Y. Zhang, B. L. West, B. Powell, G. Habets, C. Zhang, P. N. Ibrahim, P. Hirth, D. R. Artis, M. Herlyn, G. Bollag, Discovery of a selective inhibitor of oncogenic B-Raf kinase with potent antimelanoma activity. Proc. Natl. Acad. Sci. USA 105, 3041-3046 (2008).

64. T. S. Jani, J. DeVecchio, T. Mazumdar, A. Agyeman, J. A. Houghton. Inhibition of NF-kappaB signaling by quinacrine is cytotoxic to human colon carcinoma cell lines and is synergistic in combination with tumor necrosis factor-related apoptosis-inducing ligand (TRAIL) or oxaliplatin. J. Biol. Chem. 285, 19162-19172 (2010).

Acknowledgments: We acknowledge Merck KGaA (Darmstadt, Germany) for a generous gift of cetuximab. We thank L. Ailles for providing the TOP-GFP reporter construct; L. Azzolin, E. Berrino, I. Catalano, M. Cordenonsi, F. Cottino, S.M. Leto, S. Marsoni, F. Orzan, and G. Siravegna for discussion and sharing reagents; A. Ferrero, A. Mellano, A. Muratore, M. Papotti, G. Paraluppi, S. Perotti, and A. Sapino for sample acquisition; M. Buscarino and B. Martinoglio, for support with real-time PCR and Sanger sequencing; F. Maina, R. Machiorlatti and C. Zerbone for animal husbandry; R. Albano, S. Giove, L. Fontani, and L. Palmas for technical assistance; and D. Gramaglia and G. Falca for secretarial assistance. Funding: Supported by AIRC, Associazione Italiana per la Ricerca sul Cancro, 
Investigator Grants 20697 (to A.B.), 12944 (to E.M.), 20290 (to D.P.), and 18532 (to L.T.). AIRC 5x1000 grant 21091 (to A.B., E.M. and L.T). AIRC/CRUK/FC AECC Accelerator Award 22795 (to L.T.). My First AIRC Grant 19047 (to C.I.). European Research Council Consolidator Grant 724748 - BEAT (to A.B.). H2020 grant agreement no. 754923 COLOSSUS (to L.T.). H2020 INFRAIA grant agreement no. 731105 EDIReX (to A.B. and E.M.). Transcan, TACTIC (to L.T.). Fondazione Piemontese per la Ricerca sul Cancro-ONLUS, 5x1000 Ministero della Salute 2015 (to E.M. and L.T.), 2014 and 2016 (to L.T.). A.B., E.M. and L.T. are members of the EurOPDX Consortium. A.P. is the recipient of a fellowship from Fondazione Umberto Veronesi. Author contributions: B.L. was responsible for study design, biological experimentation in vitro, data interpretation, and manuscript writing. F.S. performed immunohistochemistry and analyzed and interpreted morphological data. M.P. assisted with reporter assays and RT-qPCR experiments. F.G. conducted mutational and gene expression analyses. E.R.Z. coordinated the biobank of viable CRC specimens and molecular derivatives. V.V. performed experiments with CRC organoids. G.M. designed and conducted PDX experimentation. P.A.M. designed, performed and interpreted experiments with colospheres under the supervision of A.P. and L.P. D.M. performed ChIP-seq experiments under the supervision of D.P. P.L. and C.B. provided colospheres. M.K., M.W.P. and I.H. developed, characterized and provided the Pan-HER antibody. E.M and C.I. analyzed gene expression data. D.N., I.S., T.H., A.V. and T.H. performed and interpreted WES and RNA-seq analyses under the supervision of A.S. E.E., G.A., P.N. and R.D. selected, annotated and provided clinical samples. E.G. analyzed gene expression data and supervised statistical analyses. A.B. and L.T. conceptualized the study, coordinated and supervised overall research, and wrote the manuscript. All authors have critically read, edited, and approved the final version of the manuscript. Competing interests: L.T. receives research grants from Symphogen, which has developed the Pan-HER antibody mixture used in this study. M.W.P. is, and M.K. and I.D.H. have been, employed at Symphogen and have ownership interest (including patents) in the same. L.T. also receives research grants from Servier, Pfizer, and Merus, and he is in the speakers' bureau of Eli Lilly, AstraZeneca, and Merck KGaA. Data and materials availability: Microarray data have been deposited in GEO with accession code GSE108277. RNA-seq data have been deposited in the Sequence Read Archive (SRA) with accession code EGAS00001003765. WES data have been deposited in SRA with accession code EGAS00001003764. PDX models and derivatives thereof are available for solely academic purposes from L.T. or A.B. under a material transfer agreement with the University of Torino. All data associated with this study are present in the paper or the Supplementary Materials. 


\section{FIGURE LEGENDS}

Figure 1. Residual tumors after EGFR blockade are made of slowly cycling cells with high Wnt signaling

(A) Waterfall plot of response after 3 weeks of treatment with cetuximab (20 mg/kg twice a week), compared with tumor volume at baseline, in a population of 279 PDX models ( $n=6$ or 12 mice for each bar, depending on whether initial engraftment was successful in one or two mice). Dotted lines indicate the cut-off values for arbitrarily defined categories of therapy response (14): regressions (below the lower line, $-50 \%$ ), progressive disease (above the upper line, $+35 \%$ ), and stabilizations (between the lines). Gray bars designate cases with no mutations in KRAS, NRAS, or BRAF; red, orange, and yellow bars denote cases harboring the indicated mutations. Green bars identify the 'reference collection' of responder cases selected for characterization of residual disease (all wild-type for KRAS, NRAS, and $B R A F$ ). (B) Morphometric quantitation of proliferation (Ki67 staining, left panel) and apoptosis (caspase 3 staining, right panel) in PDXs from the reference collection after treatment with vehicle (until tumors reached an average volume of $1500 \mathrm{~mm}^{3}$ ) or cetuximab (for 6 weeks). Each dot represents the average of 10 optical fields (Ki67, 40X) or 5 optical fields (caspase 3, 20X) in a section from randomly chosen tumors from vehicle-treated and cetuximab-treated mice bearing a PDX from the same original patient $(n=30)$. The plots show means \pm SD. Statistical analysis by two-tailed paired Student's $t$-test. Casp, caspase. Cetux, cetuximab. (C) Scatter plot showing GSEA (diamonds) and IPA (circles) results of differential gene expression profiles obtained by comparing PDXs treated with cetuximab with their matched vehicle-treated controls (GSE108277). Enrichments are plotted on the y axis, and their significance is plotted on the $x$ axis. Relevant gene sets related to cell cycle and metabolism are colored as shown in the figure. ES, enrichment score. (D) GSEA plots showing modulation of the EGFR inhibition signature, as observed in LGR5+ normal intestinal cells (23), in PDXs treated with cetuximab (GSE108277). EGFRi, EGFR inhibition; NES, normalized enrichment score; FDR, false discovery rate. (E) Left panel, qualitative morphometric assessment of the extent of nuclear $\beta$-catenin translocation in PDXs from the reference collection after treatment with cetuximab for 6 weeks, compared with vehicletreated counterparts. Right panels, representative images in PDXs from the reference collection after treatment with vehicle or cetuximab. Scale bar, $50 \mu \mathrm{m}$. (F) Heatmap showing expression changes for the indicated Wnt target genes in PDXs of the reference collection after treatment with cetuximab for 6 weeks, as assessed by RT-qPCR. Average gene expression, Log2R relative to vehicle-treated tumors: LGR5 0.46, $P=0.029$; LEF1 1.98, $P<0.0001$; ASCL2 0.61, $P=0.011$ by two-tailed Wilcoxon test. Benjamini-Hochberg FDR $<0.1$ for all genes. (G) Heat maps of CTNNB1 gene (encoding $\beta$ catenin) ChIP-seq signal in the indicated tumors and experimental conditions at CTNNB1 enriched genomic loci $+/-4 \mathrm{~kb}$ to the peak center. (H) Longitudinal time-lapse monitoring of $\beta$-catenin transcriptional activity in CRC0078 colospheres transduced with a TOP-GFP lentiviral construct 
containing a TCF/LEF-responsive promoter. PuraMatrix-embedded colospheres were treated with vehicle or cetuximab $(20 \mu \mathrm{g} / \mathrm{ml}$ ) in EGF-deprived medium (EGF $=0.4 \mathrm{ng} / \mathrm{ml}$ ) for 6 days. Upper panel, representative images (scale bar, $50 \mu \mathrm{m}$ ); lower panel, morphometric quantitation of GFP fluorescence. Values are the means \pm SEM from one experiment $(n=18$ for vehicle-treated colospheres and 20 for cetuximab-treated colospheres). Statistical analysis by two-way ANOVA.

Figure 2. Cancer cells that withstand EGFR inhibition express markers of the Paneth cell lineage

(A) Heatmap showing expression changes for the indicated LRC markers in PDXs of the reference collection after treatment with cetuximab (20 mg/kg twice a week for 6 weeks), as assessed by RTqPCR. Average gene expression, Log2R relative to vehicle-treated tumors: $B M I 10.57, P=0.0002$; HOPX 1.41, $P<0.0001$; LRIG1 0.42, $P=0.006$; TERT -1.41, $P=0.0071$ by two-tailed Wilcoxon test. Benjamini-Hochberg FDR $<0.1$ for all genes. (B) GSEA plot showing positive modulation of the Mex3ahigh/Lgr5-high signature (29) in PDXs treated with cetuximab (GSE108277). NES, normalized enrichment score; FDR, false discovery rate. (C) GSEA plot showing upregulation of a manually curated subset of secretory/Paneth cell genes in PDXs treated with cetuximab (GSE108277). (D) Heatmap showing expression changes for the indicated secretory/Paneth-cell markers in PDXs of the reference collection after treatment with cetuximab, as assessed by RT-qPCR. Average gene expression, Log2R relative to vehicle-treated tumors: DEFA5 4.03, $P<0.0001 ; D L L 11.12, P=0.002$; ATOH1 1.26, $P<0.0001$; DEFA6 2.95, $P=0.0001$; GFI1 1.04, $P=0.0006$ by two-tailed Wilcoxon test. Benjamini-Hochberg FDR $<0.1$ for all genes. (E) Morphometric quantitation of DEFA5 and DEFA6 protein expression changes in PDXs of the reference collection after treatment with cetuximab. Each bar represents the change of average protein expression in 5 optical fields (20X) in a section from randomly chosen tumors from cetuximab-treated mice compared with a tumor from matched vehicletreated mice ( $n=5$ for each bar). Representative images are also shown. $P=0.028$ for DEFA5 and 0.0131 for DEFA6 by two-tailed paired Student's $t$-test. Scale bar, $100 \mu \mathrm{m}$. Cetux, cetuximab. (F) Expression of secretory/Paneth cell markers in a dataset of mCRC PDXs annotated for response to cetuximab (GSE76402). Each dot represents the average expression of the secretory/Paneth cell signature metagene (ATOH1, GFI1, SOX9, XBP1, DEFA5, DEFA6, LYZ, SPINK4, DLL1, DLL4) in individual tumors. Log2R relative to the dataset median (red line). $P<0.0001$ by two-tailed unpaired Student's t-test. (G) Progression-free survival of patients with CRC metastases treated with cetuximab monotherapy (34), divided into two groups using the median signal intensity of the secretory/Paneth cell signature metagene as a cutoff ( $n=40$ patients with high signature and 40 patients with low signature). Statistical analysis by Cox regression. 


\section{Figure 3. Cetuximab inactivates YAP}

(A) Correlation between gene expression changes induced by cetuximab (20 mg/kg twice a week for 6 weeks) in CRC PDXs and those induced by YAP knock-out (YAP $\Delta / \Delta)$ in the normal mouse intestine (35). Left panel, scatter plot of gene expression changes. Right panel, Pearson's correlation of differentially regulated genes. Cetux, cetuximab. (B) GSEA plot showing the regulation of YAPinducible target genes (36) in PDXs treated with cetuximab. NES, normalized enrichment score; FDR, false discovery rate. (C) Left panels, qualitative morphometric assessment of YAP total and nuclear expression in PDXs from the reference collection after treatment with cetuximab, compared with vehicle-treated counterparts. Right panels, representative images in PDXs from the reference collection after treatment with vehicle (until tumors reached an average volume of $1500 \mathrm{~mm}^{3}$ ) or cetuximab. Scale bar, $50 \mu \mathrm{m}$. Expr, expression; ref, reference. (D) Measurement of YAP transcriptional activity in DiFi and HCA46 cell lines after treatment for $48 \mathrm{~h}$ with vehicle (DMSO), cetuximab $(20 \mu \mathrm{g} / \mathrm{ml})$, selumetinib $(1 \mu \mathrm{M})$, or dactolisib $(250 \mathrm{nM})$. Luciferase activity was normalized against reporter plasmid concentrations as determined by DNA qPCR and expressed as arbitrary units (A.U.). Three independent experiments were performed in biological quadruplicates $(n=12)$. The plots show means \pm SD. Statistical analysis by one-way ANOVA followed by Benjamini, Krieger and Yekutieli FDR correction. (E) Effect of doxycycline-regulated YAP activation, with or without concomitant EGFR inhibition, in established DiFi xenografts. Left panel, tumor volume changes after 3 weeks of treatment with cetuximab $(20 \mathrm{mg} / \mathrm{kg}$, intraperitoneal injection twice a week) and/or doxycycline $(50 \mathrm{mg} / \mathrm{kg}$ daily oral gavage). Right panel, tumor volume changes after 4 weeks from treatment suspension. $n=7$ animals per each treatment arm. Statistical analysis by one-way ANOVA followed by Benjamini, Krieger and Yekutieli FDR correction (left panel) or two-tailed Wilcoxon test (right panel). Control, doxycycline vehicle + cetuximab vehicle; Doxy, doxycycline; Combo, doxycycline + cetuximab.

Figure 4. Acquisition of the Paneth cell-like phenotype in residual cells is regulated by YAP inactivation

(A) RT-qPCR analysis of the indicated secretory/Paneth-cell markers in DiFi and HCA46 cells and in CRC0078 colospheres treated with cetuximab, selumetinib, or dactolisib for $72 \mathrm{~h}$. Independent experiments ( $n=3$ for DiFi and CRC0078; $n=4$ for HCA46) were performed in technical triplicates. In some experiments, controls with untreated cells were repeated. The plots show means \pm SD. The effects of cetuximab and selumetinib were positively correlated (DiFi, $r=0.857$; HCA46, $r=0.963$; CRC0078, $r=0.984$, Pearson's correlation coefficient), whereas dactolisib-induced transcriptional changes tended to negatively correlate with those triggered by cetuximab ( $\mathrm{DiFi}, r=-0.592$; HCA46, $r$ $=-0.104$; CRC0078, $r=-0.905$ ). Cetux, cetuximab. (B) RT-qPCR analysis of the indicated secretory/Paneth-cell genes in DiFi and HCA46 cell lines transduced with a pLVX-control vector (mock), the constitutively active YAP variant (YAP-5SA), or wild-type YAP (YAP-WT), and treated with 
cetuximab $(20 \mu \mathrm{g} / \mathrm{ml}$ ) for $72 \mathrm{~h}$. Independent experiments ( $n=3$ for HCA46 and $n=4$ for DiFi) were performed in technical triplicates. In some experiments, controls with untreated cells were repeated. The plots show means \pm SD. Statistical analysis by one-way ANOVA followed by Benjamini, Krieger and Yekutieli FDR correction. Stars next to DEFA5 and DEFA6 gene names indicate loss of basal expression in the YAP-5SA experimental group, which prevented statistical comparison against mock controls for these genes. In the DiFi YAP-WT group, DEFA5 and DEFA6 expression was above detection in one replicate. (C) RT-qPCR analysis of the indicated secretory/Paneth-cell genes in established DiFi xenografts transduced with doxycycline-inducible YAP-5SA and treated with cetuximab (20 mg/kg, 2 administrations), doxycycline (50 mg/kg daily), or both for 1 week. Four samples for each condition were analyzed in technical triplicates. The plots show means \pm SD. Statistical analysis by one-way ANOVA followed by Benjamini, Krieger and Yekutieli FDR correction. Doxy, doxycycline.

Figure 5. Combined EGFR/PI3K inhibition unleashes apoptosis and reduces residual disease in vitro but has suboptimal activity in vivo

(A) Luciferase-based evaluation of apoptosis (assessed by caspase 3/7 activity) in DiFi and HCA46 cell lines treated for $24 \mathrm{~h}$ with the indicated drugs at the following concentrations: cetuximab, $20 \mu \mathrm{g} / \mathrm{ml}$; BMS345541, ruxolitinib, selumetinib, PLX4720, alpelisib, $1 \mu \mathrm{M}$; dactolisib, $250 \mathrm{nM}$; everolimus, $50 \mathrm{nM}$; pictilisib, $100 \mathrm{nM}$; MK2206, $0.5 \mu \mathrm{M}$; buparlisib, $0.5 \mu \mathrm{M}$. To make sure that the different drugs would be used at biologically relevant concentrations, we mined a publicly available, large-scale data set reporting the sensitivity of several hundred cancer cell lines to hundreds of drugs (59); we then extracted the minimum $\mathrm{IC}_{50}$ of the specific inhibitors, and used them at an least two-fold higher concentration. The elected drug concentrations have proven to be active in various cell lines also in independent studies: (60) (ruxolitinib, alpelisib, pictilisib, buparlisib, MK2206); (61) (dactolisib, selumetinib); (62) (everolimus); (63) (PLX4720); and (64) (BMS345541). Results represent the means \pm SD of 2 independent experiments performed in biological quintuplicates $(n=10)$. The effect of different PI3K/AKT inhibitors is indicated by red bars. The targets of each drug are specified. Statistical analysis (combinatorial treatment versus cetuximab alone) by one-way ANOVA followed by Benjamini, Krieger and Yekutieli FDR correction. Cetux, cetuximab. (B) Representative confocal images of PuraMatrix-embedded CRC0078 colospheres treated for 2 weeks with vehicle (DMSO), cetuximab (10 $\mu \mathrm{g} / \mathrm{ml}$ ), selumetinib (300 $\mathrm{nM})$, or dactolisib (100 $\mathrm{nM}$ ), alone or in combination, prior to fixation and staining for F-actin (green) and DNA (blue). (C) Growth curves of PuraMatrix-embedded CRC0078 colospheres treated with cetuximab $(30 \mu \mathrm{g} / \mathrm{ml})$, selumetinib $(1 \mu \mathrm{M})$, or dactolisib $(300 \mathrm{nM})$, alone or in combination, for 21 days and then kept without drugs for additional 28 days. Time-lapse imaging was carried out by bright field microscopy, and individual equatorial areas were measured by Image $\mathrm{J}$ software. Average time-dependent area variation is represented as fold change relative to area value 
at day 21 . Results are the means \pm SEM of at least 15 colospheres for each experimental condition. Statistical analysis by two-way ANOVA (calculated after drug withdrawal) followed by Benjamini, Krieger and Yekutieli FDR correction: cetuximab + selumetinib versus cetuximab alone, $P=0.0138$; cetuximab + dactolisib versus cetuximab alone, $P<0.0001$. (D) Tumor volume changes in PDXs treated with the indicated modalities for 4 weeks. Cetuximab, $20 \mathrm{mg} / \mathrm{Kg}$ (intraperitoneal injection twice a week); dactolisib, $35 \mathrm{mg} / \mathrm{Kg}$ (daily oral gavage); alpelisib, $25 \mathrm{mg} / \mathrm{Kg}$ (daily oral gavage). Dots represent volume changes of individual mice, and plots show the means \pm SD for each treatment arm. $n=4$ to 14 animals per each treatment arm. For CRC0069, CRC0096, CRC0252, CRC0322, CRC0542, and CRC0743, statistical analysis was performed by two-tailed unpaired Welch's $t$-test. For CRC0078, statistical analysis was performed by one-way ANOVA followed by Benjamini, Krieger and Yekutieli FDR correction. Tumor variation outliers were excluded using the ROUT method (GraphPad Prism). Pre- and end-of-treatment tumor volumes in individual mice are reported in fig. S19.

Figure 6. EGFR blockade leads to increased HER2/HER3 signaling in PDXs and in patients

(A) RT-qPCR analysis of HER2 and HER3 expression in DiFi and HCA46 cell lines transduced with either a pLVX-control vector (mock) or the constitutively active YAP variant (YAP-5SA) and treated with cetuximab $(20 \mu \mathrm{g} / \mathrm{ml})$ for $48 \mathrm{~h}$. Independent experiments ( $n=4$ for DiFi and $n=3$ for HCA46) were performed in technical triplicates. The plots show means \pm SD. Statistical analysis by one-way ANOVA followed by Benjamini, Krieger and Yekutieli FDR correction. (B) RT-qPCR analysis of HER2 and HER3 expression in established DiFi xenografts transduced with doxycycline-inducible YAP-5SA and treated with cetuximab (20 mg/kg, 1 administration), doxycycline (50 mg/kg daily), or both for 1 week. Four samples for each condition were analyzed in technical triplicates. The plots show means \pm SD. Statistical analysis by one-way ANOVA followed by Benjamini, Krieger and Yekutieli FDR correction. Cetux, cetuximab; Doxy, doxycycline. (C) Heatmap showing expression changes for HER2 and HER3 in PDXs of the reference collection, after treatment with cetuximab for 6 weeks $(20 \mathrm{mg} / \mathrm{kg}$ twice a week), as assessed by RT-qPCR. Average gene expression, Log2R relative to vehicle-treated tumors: HER2 0.29, $P=0.029$; HER3 0.31, $P=0.014$ by two-tailed Wilcoxon test. Benjamini-Hochberg FDR < 0.1 for both genes. (D) Heatmap showing expression changes for HER2, HER3, DEFA5 and ATOH1 in tumors from patients after 4 weeks of treatment with Sym004, as assessed by RT-qPCR. Changes in the sum of diameters of target lesions, as per RECIST criteria, are indicated as tumor variations. (E) Expression of DEFA5 protein in the treatment-naïve sample and on-treatment biopsy of patient MPP192-5. Scale bar, $20 \mu \mathrm{m}$. IHC, immunohistochemistry (F) Western blot analysis of the indicated phosphoproteins in DiFi and HCA46 cells treated for 10 min with BTC at the indicated concentrations. Total proteins were used for normalization; vinculin was used as a loading control. Western blots for total proteins were run with the same lysates as those used for anti-phosphoprotein detection but on different gels. The images shown are representative of 2 experiments on independent biological 
replicates. P-EGFR, phospho-EGFR; P-HER2, phospho-HER2; P-HER3, phospho-HER3; P-ERK, phospho-ERK; P-AKT, phospho-AKT. (G) Quantitation of cell number (assessed by ATP content) in DiFi and HCA46 cell lines treated for $72 \mathrm{~h}$ with the indicated antibodies at the indicated concentrations in the absence or presence of $10 \mathrm{ng} / \mathrm{ml} \mathrm{BTC}$. Results represent the means \pm SD of 2 independent experiments performed in biological triplicates $(n=6)$. Statistical analysis by two-way ANOVA. (H) Morphometric quantitation of phospho-HER2 and phospho-HER3 immunoreactivity in PDXs from the reference collection after treatment with vehicle (until tumors reached an average volume of $1500 \mathrm{~mm}^{3}$ ) or cetuximab (for 6 weeks). Each dot represents the average of 10 optical fields (40X) in a section from randomly chosen tumors from vehicle-treated and cetuximab-treated mice bearing a PDX from the same original patient $(n=30)$. The plots show means \pm SD. Statistical analysis by ratio paired $t$-test. Scale bar, $50 \mu \mathrm{m}$.

Figure 7. Targeting HER2/HER3 together with EGFR provides long-term therapeutic benefits in PDX trials

(A) Tumor volume changes in PDXs treated with the indicated modalities for 4 weeks. Cetuximab, 20 $\mathrm{mg} / \mathrm{Kg}$ (intraperitoneal injection twice a week); Pan-HER, $60 \mathrm{mg} / \mathrm{Kg}$ (intraperitoneal injection twice a week). Dots represent volume changes of individual mice, and plots show the means \pm SD for each treatment arm. $n=5$ to 12 animals per each treatment arm. Statistical analysis by two-tailed unpaired Welch's $t$-test. Tumor variation outliers were excluded using the ROUT method (GraphPad Prism). Cetux, cetuximab. (B) Kaplan-Meier survival curves in PDXs after discontinuation of the indicated treatments. $n=5$ to 10 animals per each treatment arm. Statistical analysis by Log-rank (Mantel-Cox) test. (C) Tumor volume changes in PDXs treated with the indicated modalities for 4 weeks. $n=5$ animals per each treatment arm. Statistical analysis as in A. (D) Kaplan-Meier survival curves in PDXs after discontinuation of the indicated treatments. $n=5$ animals per each treatment arm. Statistical analysis as in B. (E) Morphometric quantitation of the phosphorylation of the indicated proteins in PDXs CRC0322 and CRC0542 after treatment with vehicle (until tumors reached an average volume of 1500 $\mathrm{mm}^{3}$ ), cetuximab (5 weeks), or Pan-HER (5 weeks). At the end of treatment, 3 tumors from 3 different mice were explanted and subjected to immunohistochemical analysis. Each dot represents the value measured in one optical field (40x), with 2 to 10 optical fields per tumor depending on the extent of section area ( $n=16$ to 30 ). The plots show means \pm SD. Statistical analysis by one-way ANOVA followed by Benjamini, Krieger and Yekutieli FDR correction. P-S6, phospho-S6; P-ERK, phosphoERK; P-HER2, phospho-HER2; P-HER3, phospho-HER3. Representative images for phospho-S6 and phospho-ERK immunoreactivity in CRC0542 are shown in fig. S25. 
A

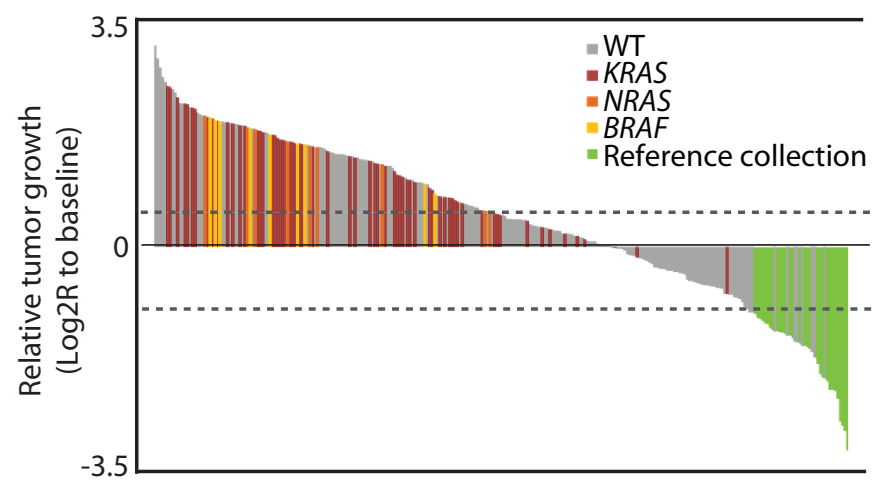

C

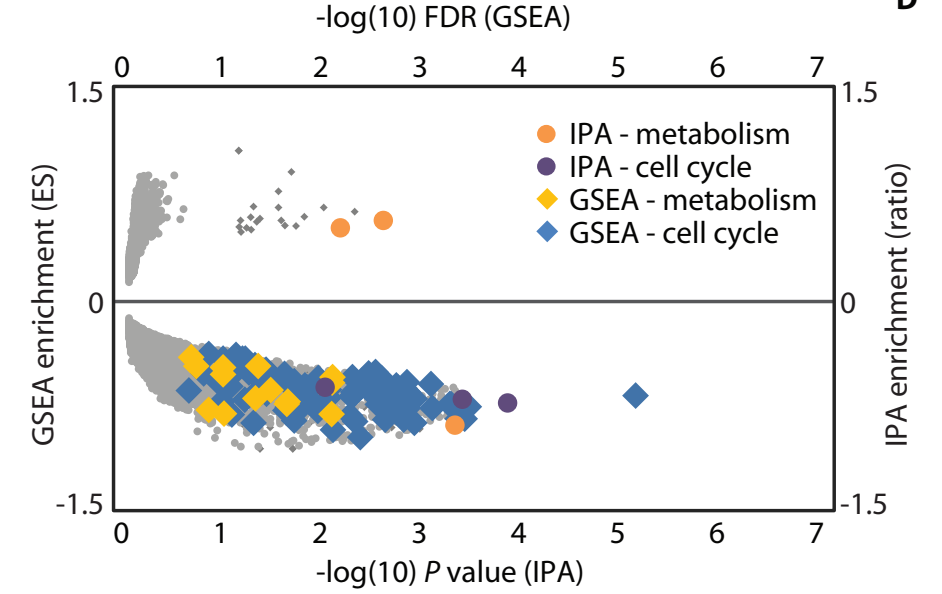

E

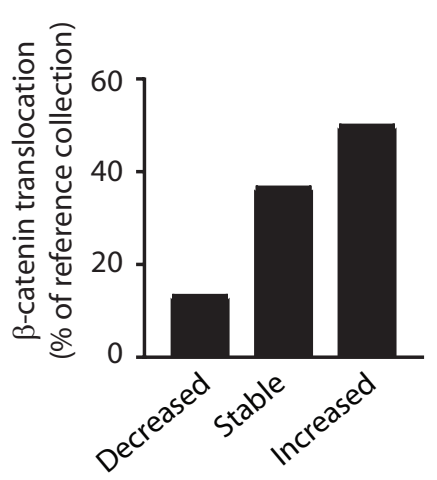

B
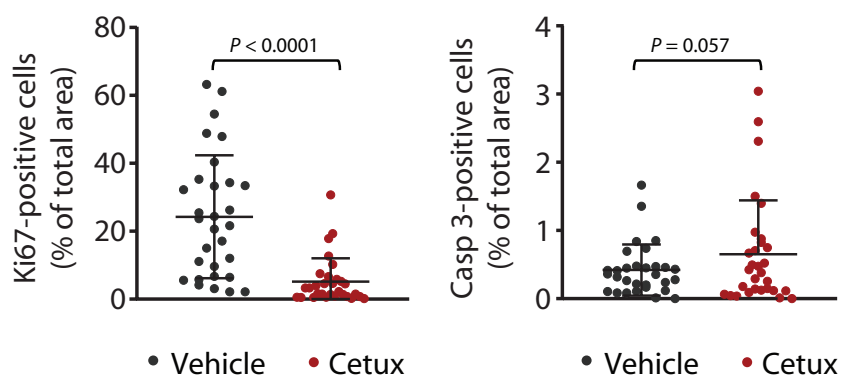

D
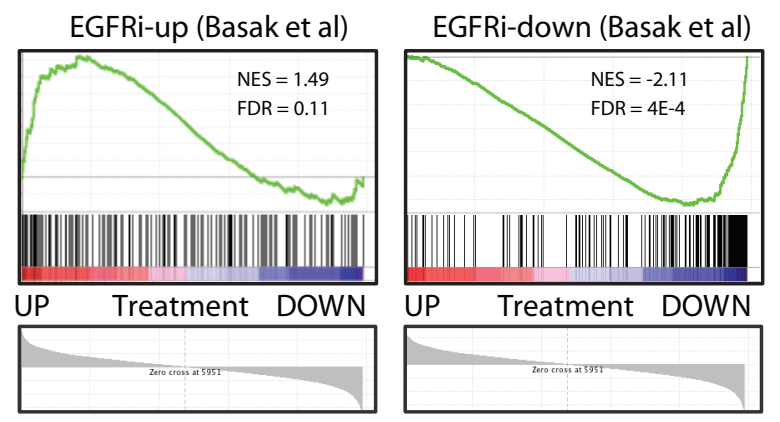

F

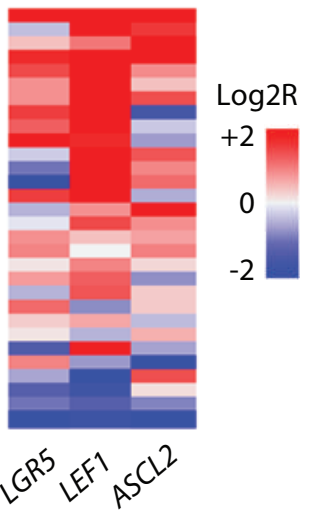

G

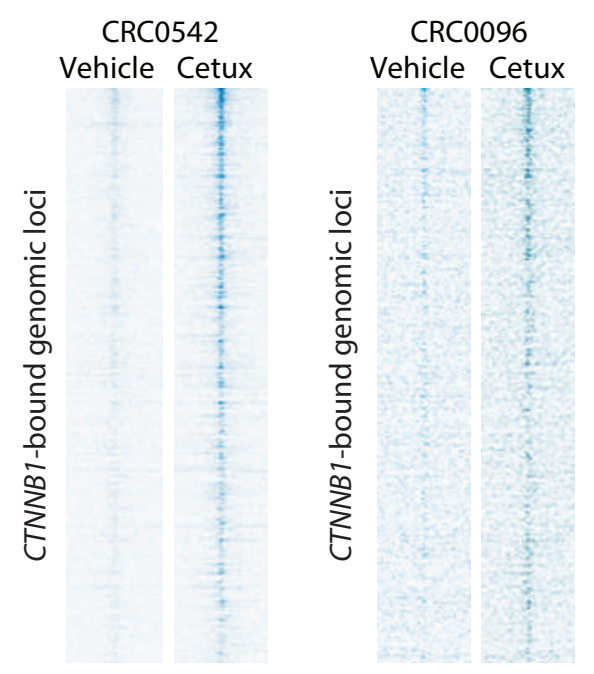

H
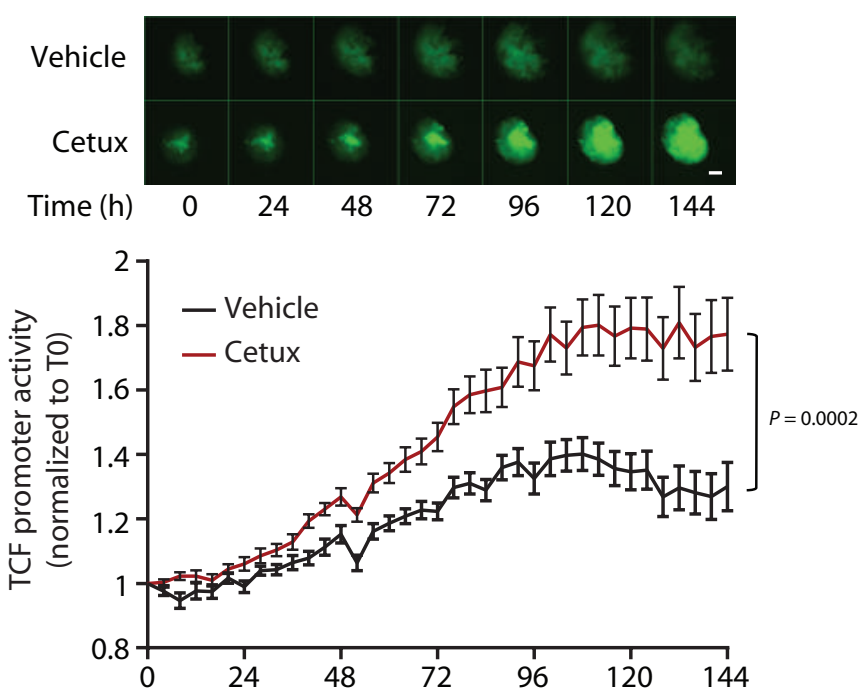
A

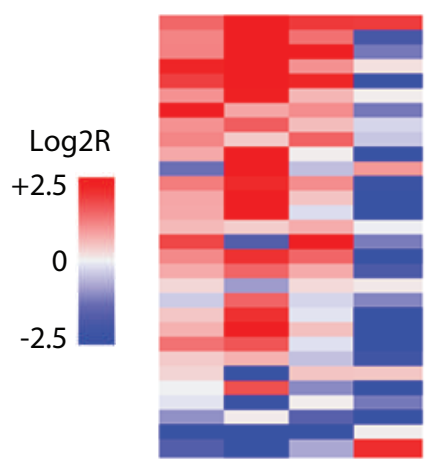

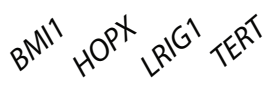

D

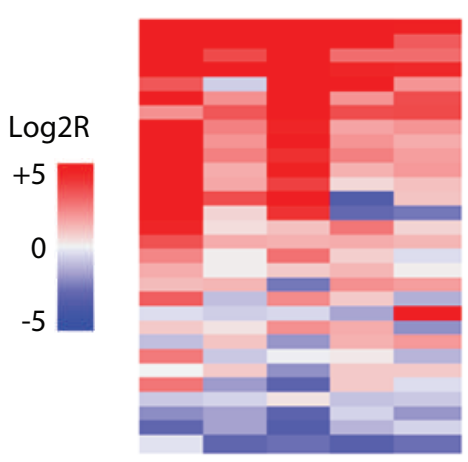

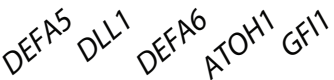

$\mathbf{F}$

PDXs (GSE76402)

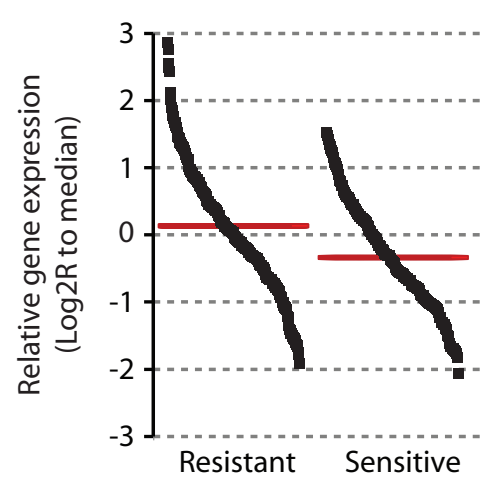

G

Patients (GSE5851)

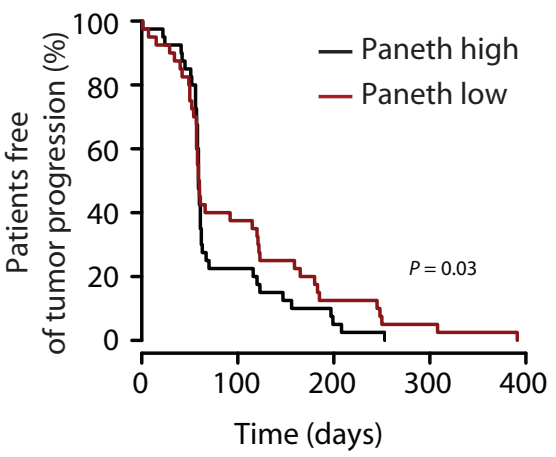

B

MEX3A-high/LGR5-high signature (Barriga et al)

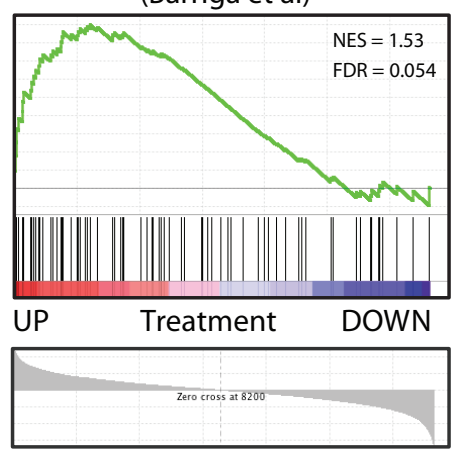

Secretory/Paneth cell markers

(manually curated signature)

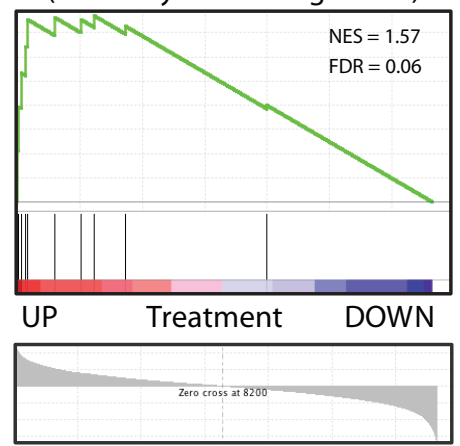

E

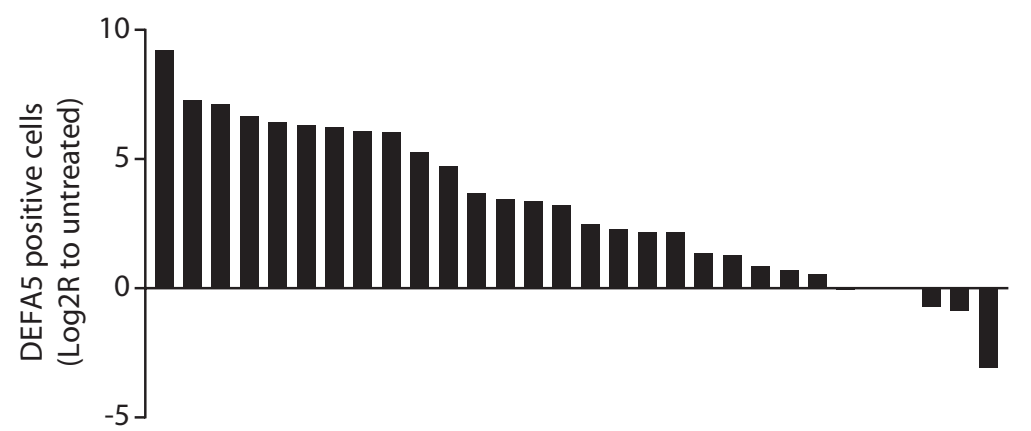

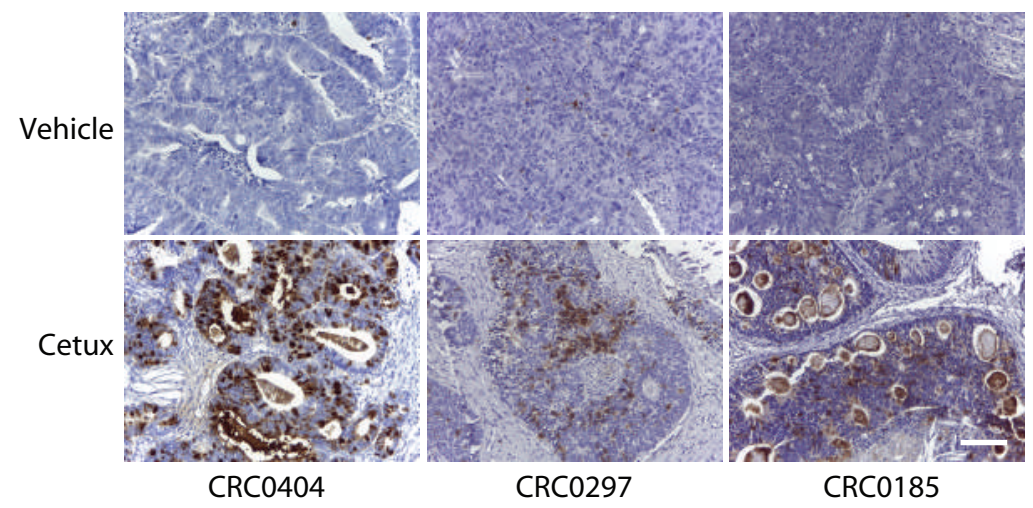
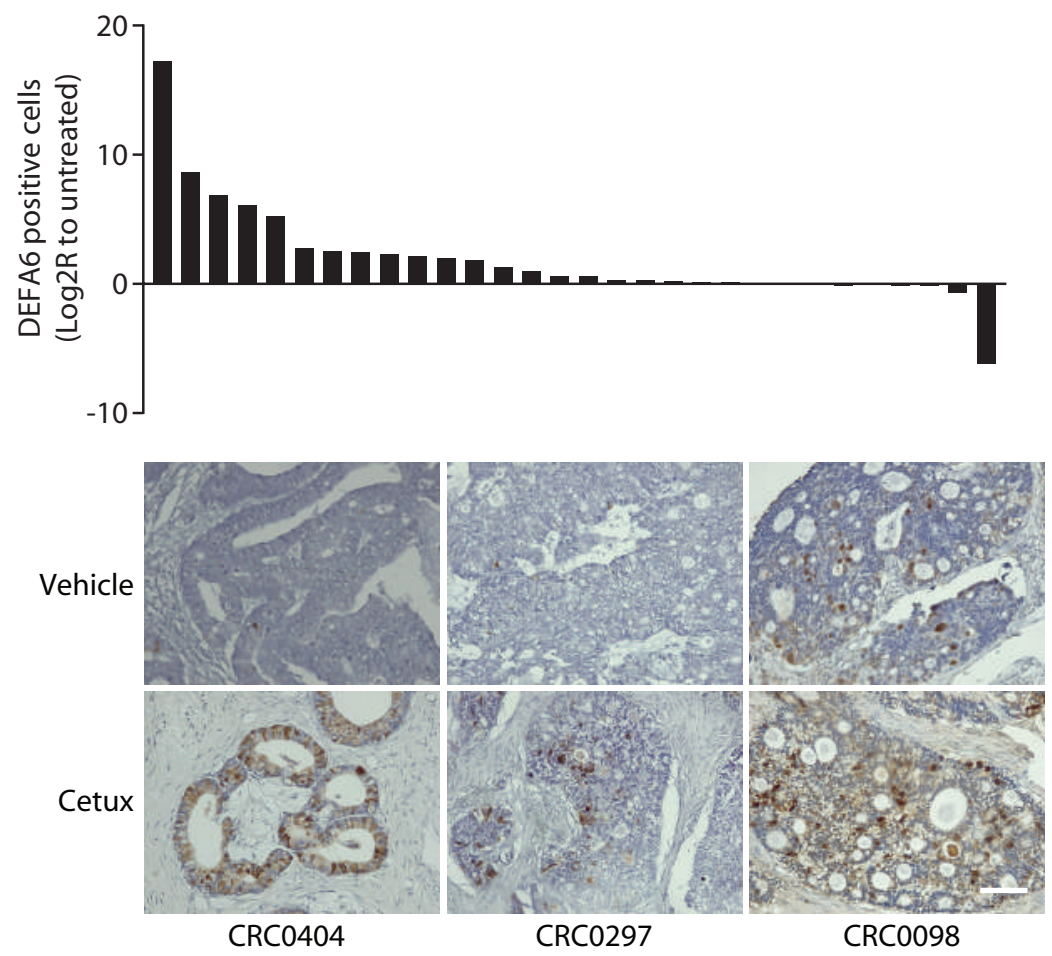

Lupo et al., Figure 2 


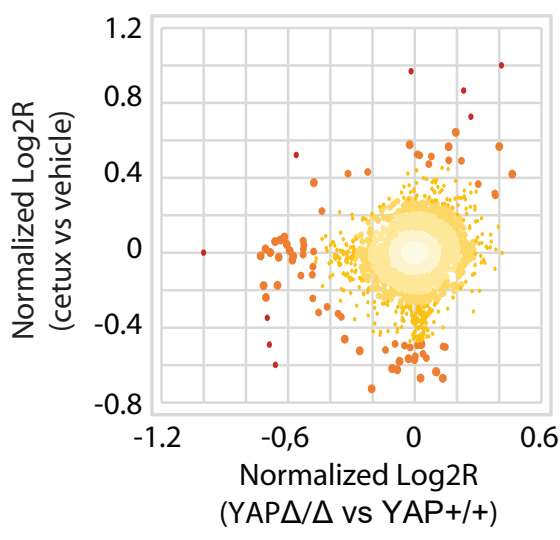

C
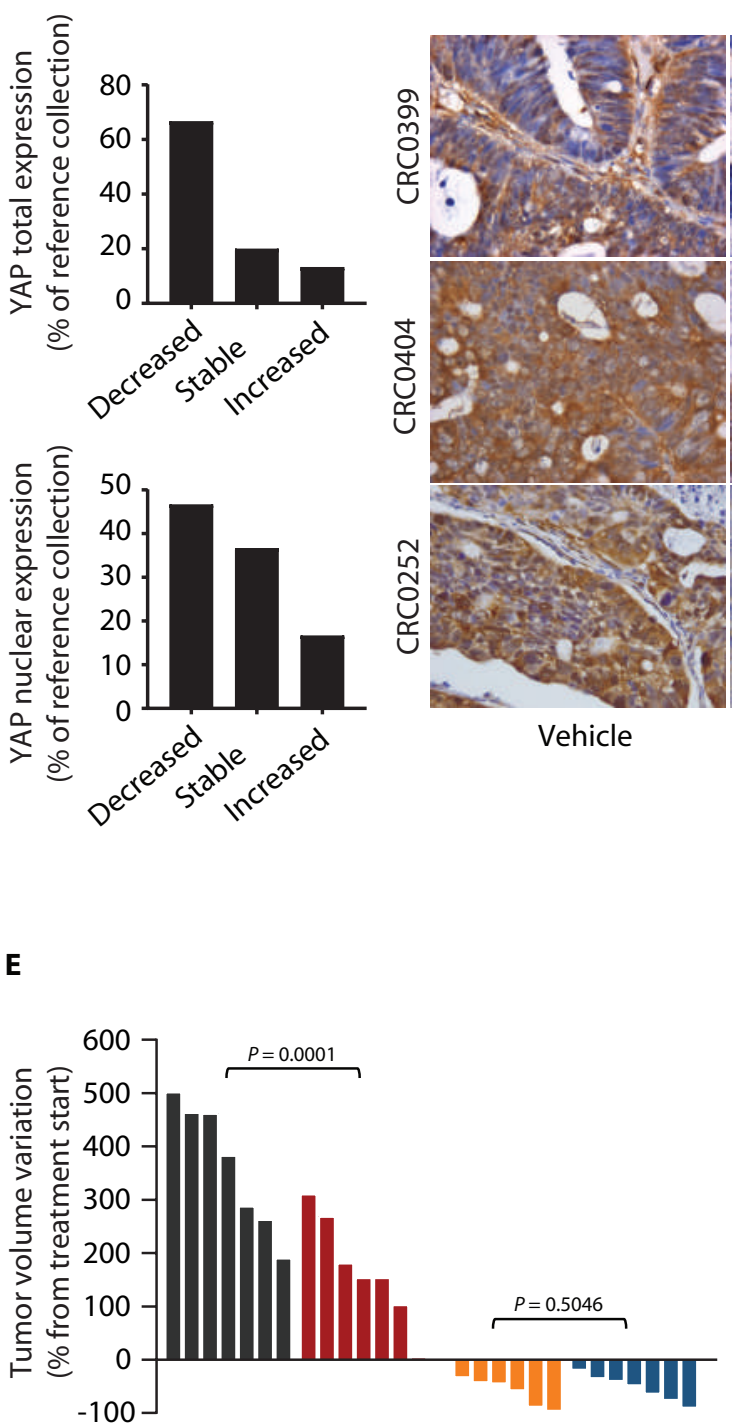
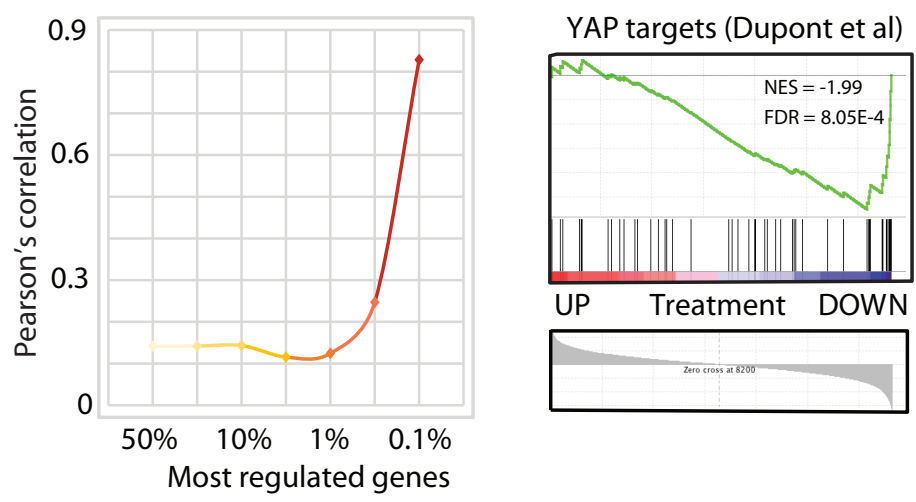

D
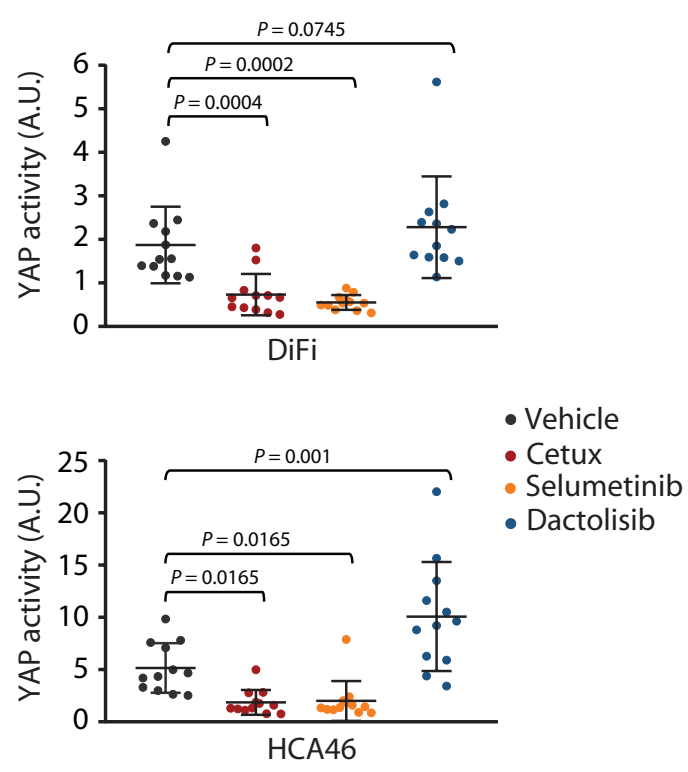
A
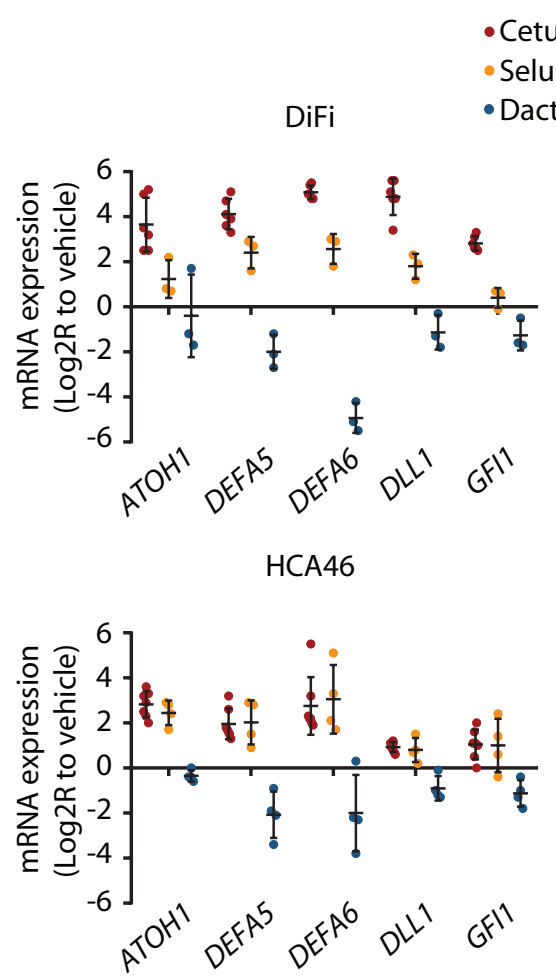

CRC0078 colospheres

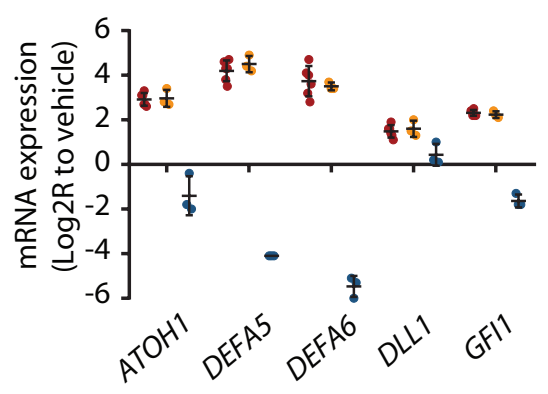

B

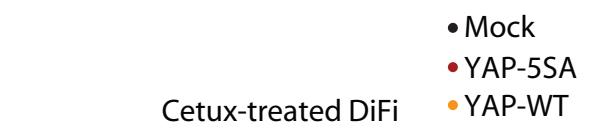

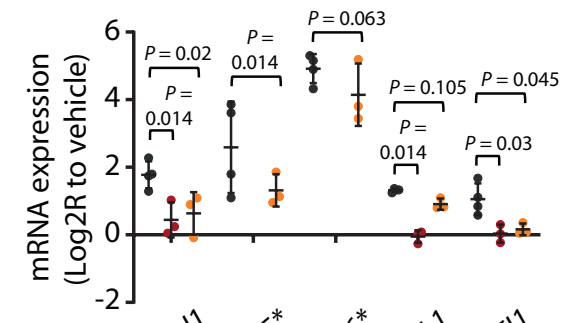

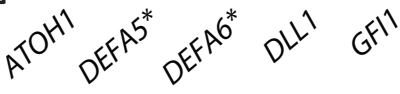

C

Cetux-treated DiFi xenografts

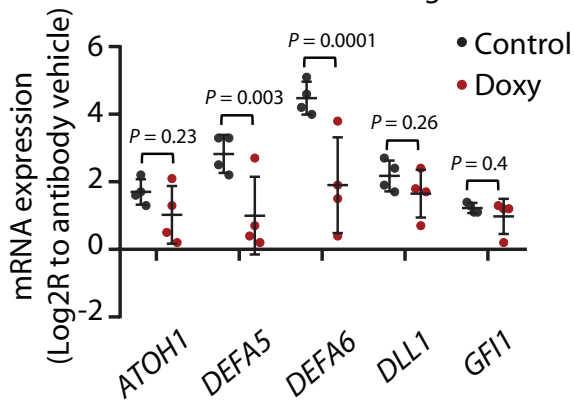




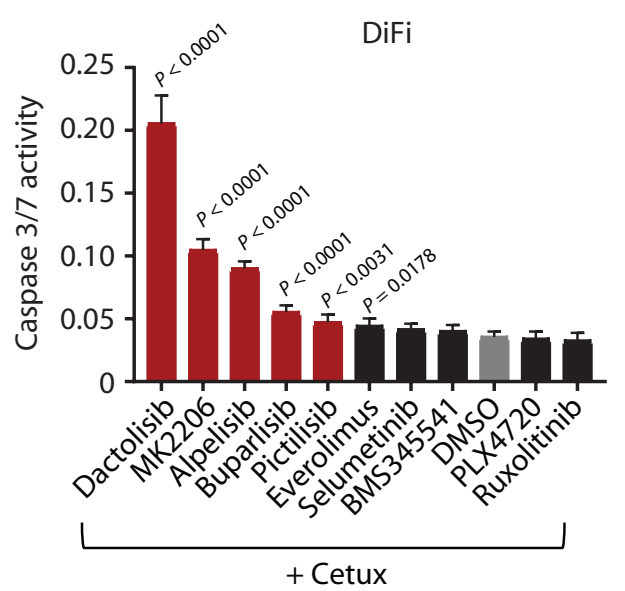

B

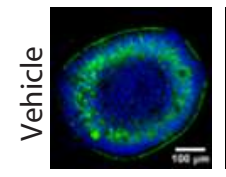

CRC0078 (colospheres)

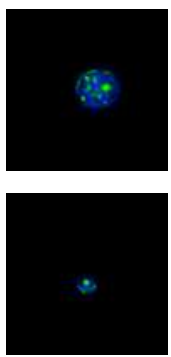

Vehicle

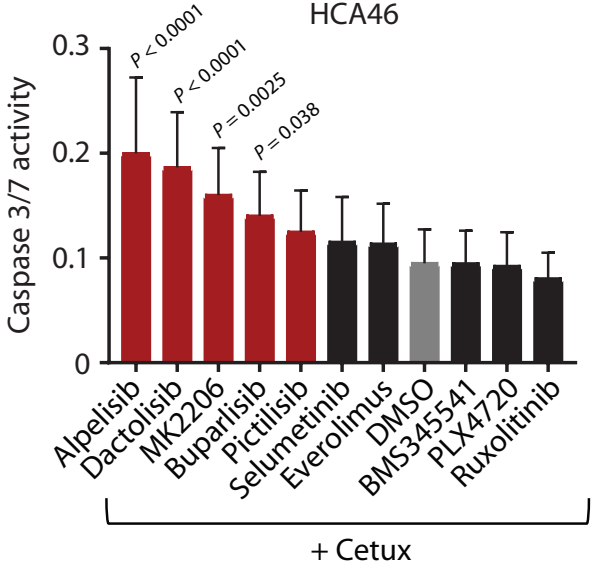

C

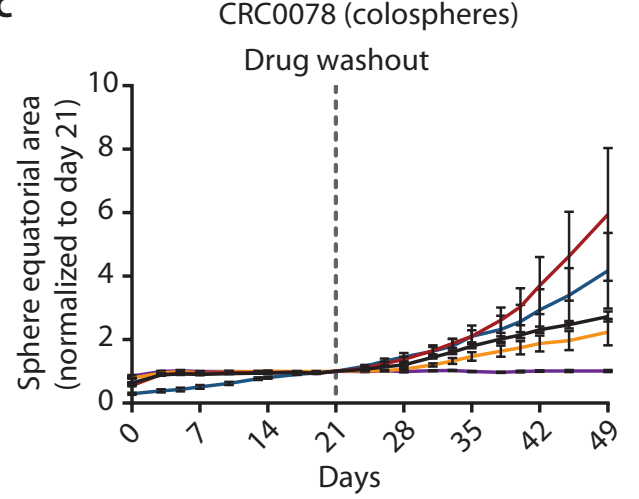

Drug targets:

Alpelisib: PI3K $\alpha$

Dactolisib: PI3K/mTOR MK2206: AKT

Buparlisib: pan-PI3K

Pictilisib: $\mathrm{PI} 3 \mathrm{~K} \alpha / \delta$

Everolimus: mTOR

PLX4720: BRAF

Selumetinib: MEK1

Ruxolitinib: JAK1/2

BMS345541: IKB/IKK
- Cetux

- Selumetinib

- Dactolisib

- Cetux + selumetinib

- Cetux + dactolisib
D
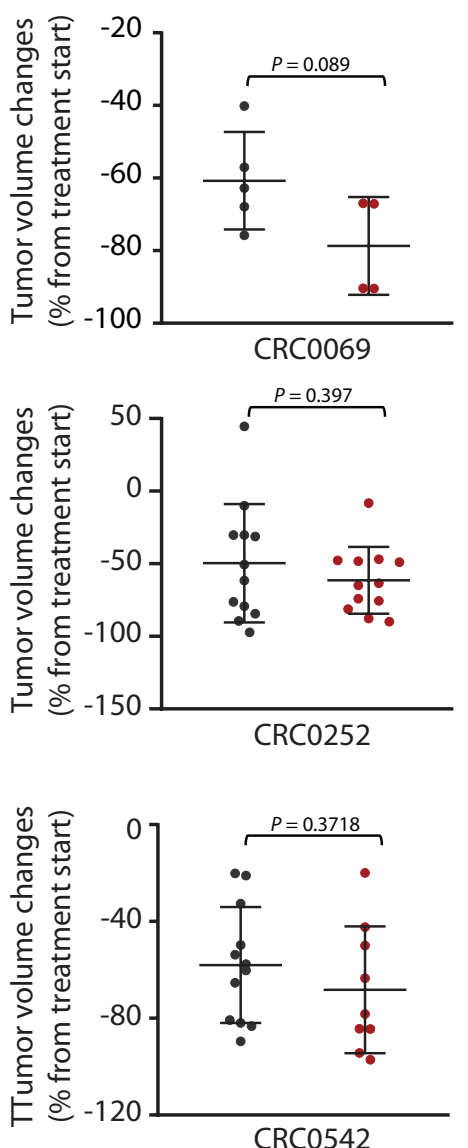
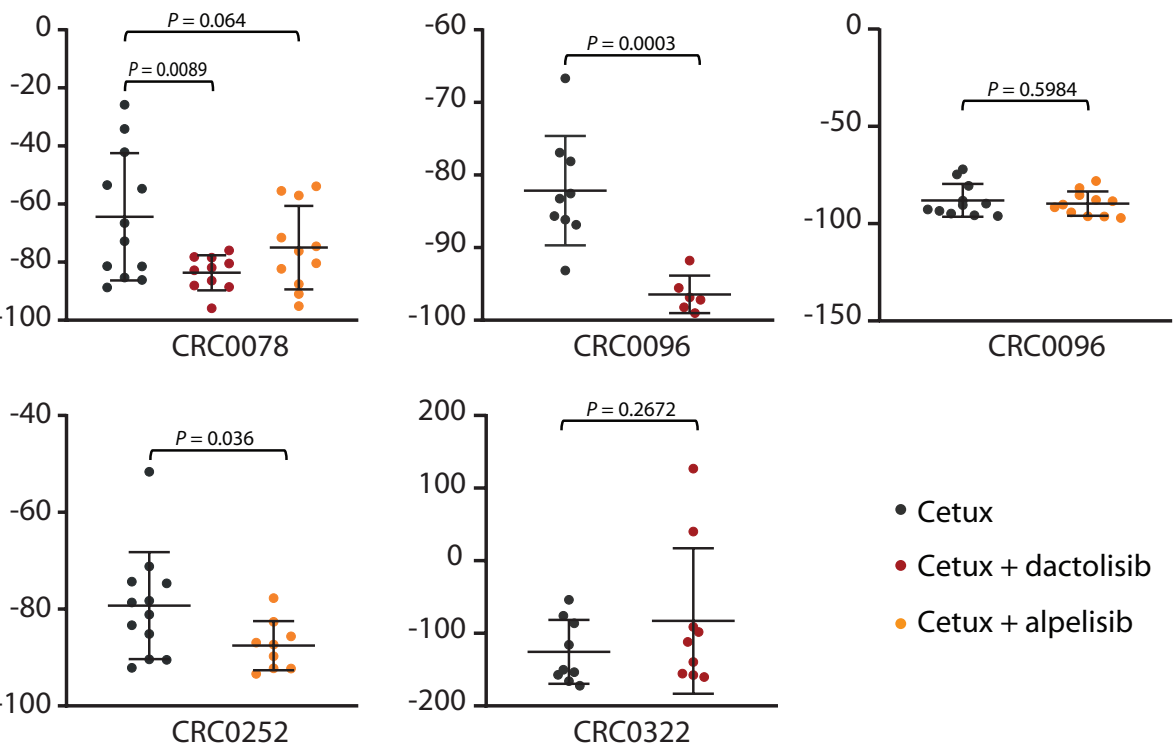

- Cetux

- Cetux + dactolisib

- Cetux + alpelisib
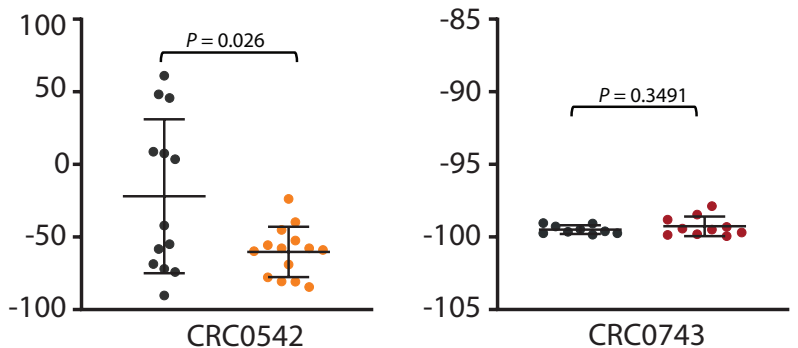
Cetux-treated DiFi

Cetux-treated HCA46

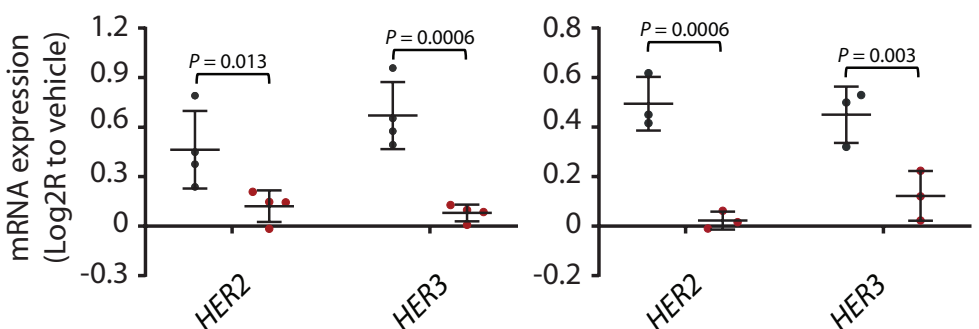

C

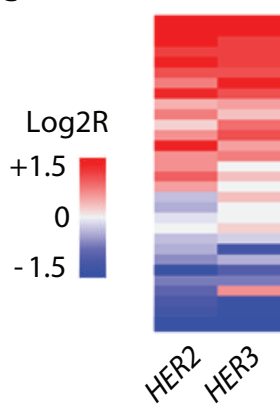

D

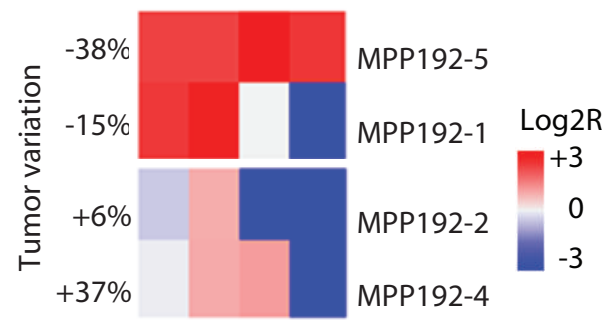

$\mathbf{F}$

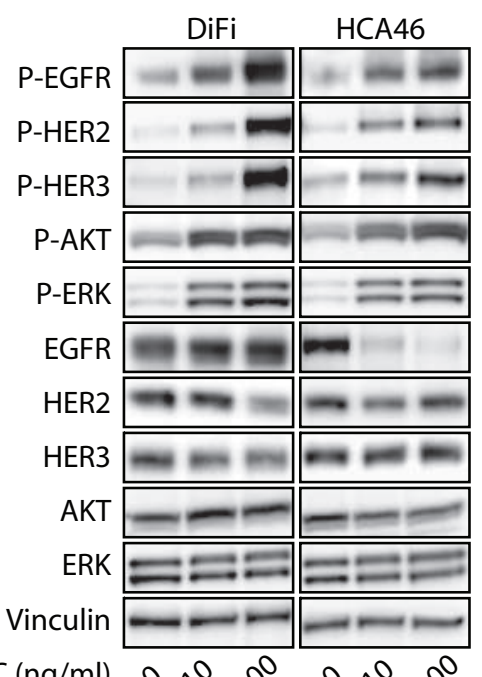

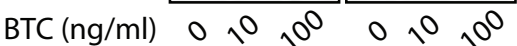

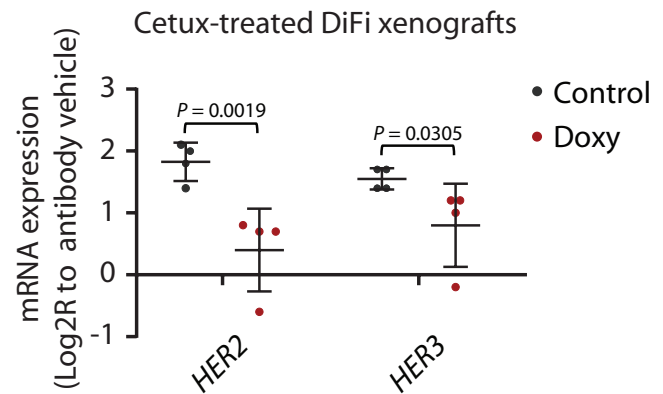

E

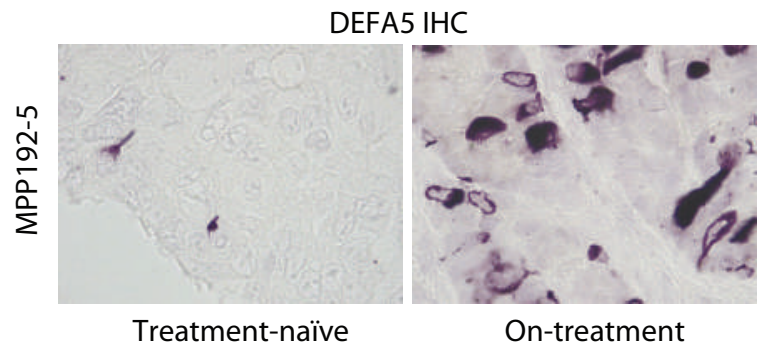

DiFi

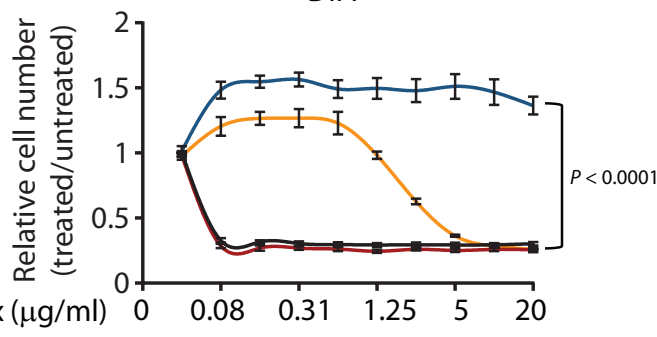

$\begin{array}{lllllllll}\text { Pan-HER }(\mu \mathrm{g} / \mathrm{ml}) & 0 & 0.23 & 0.94 & 3.75 & 15 & 60 & \text { - Cetux }\end{array}$

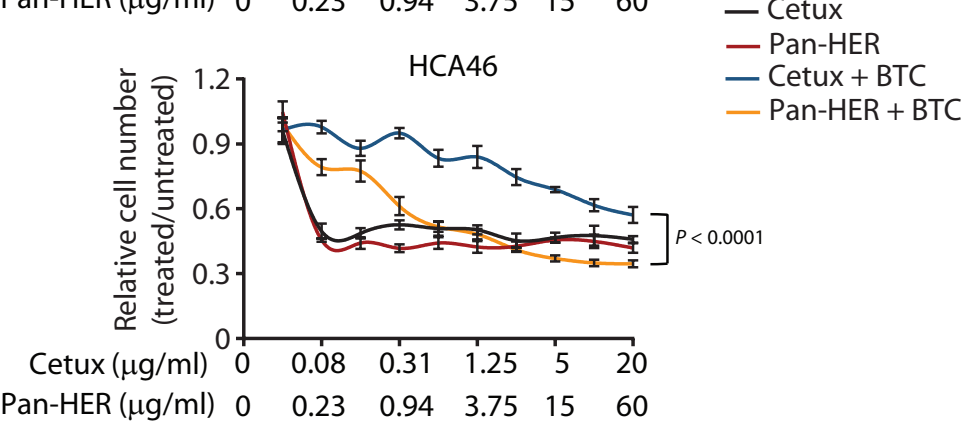

H
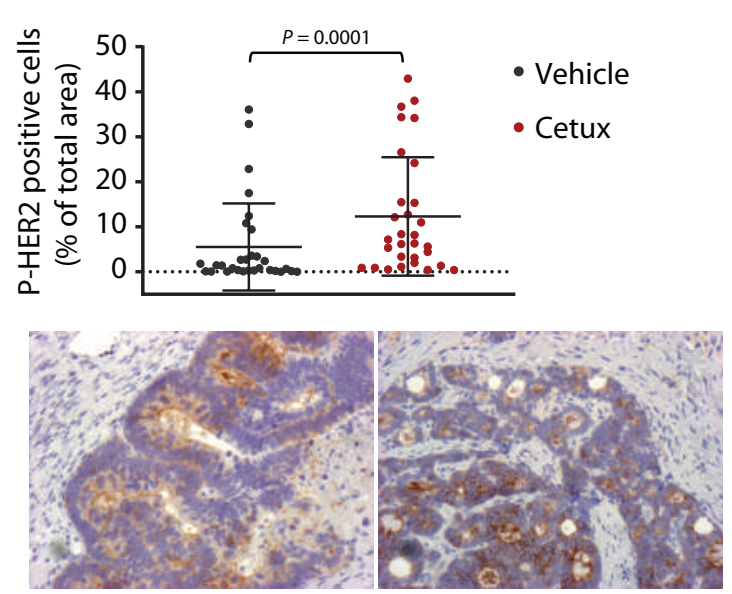

Vehicle
Cetux

CRC0322
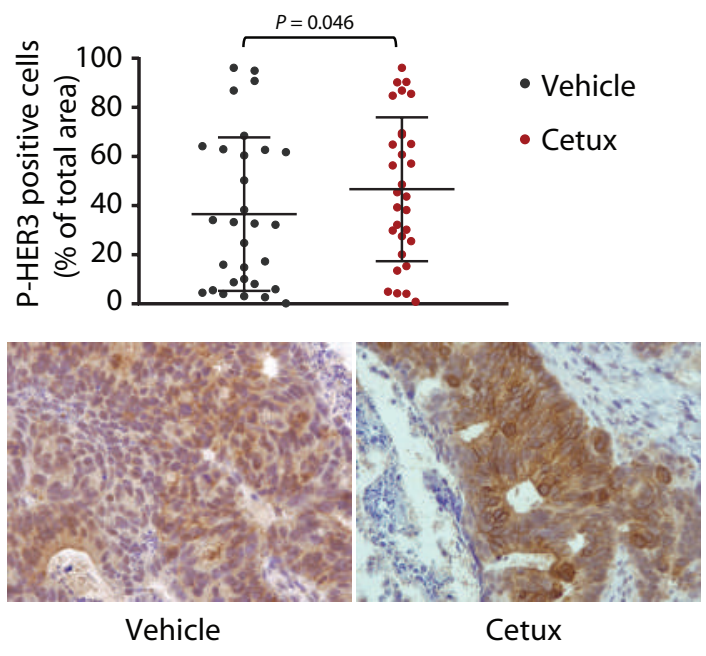

CRC0542 
A

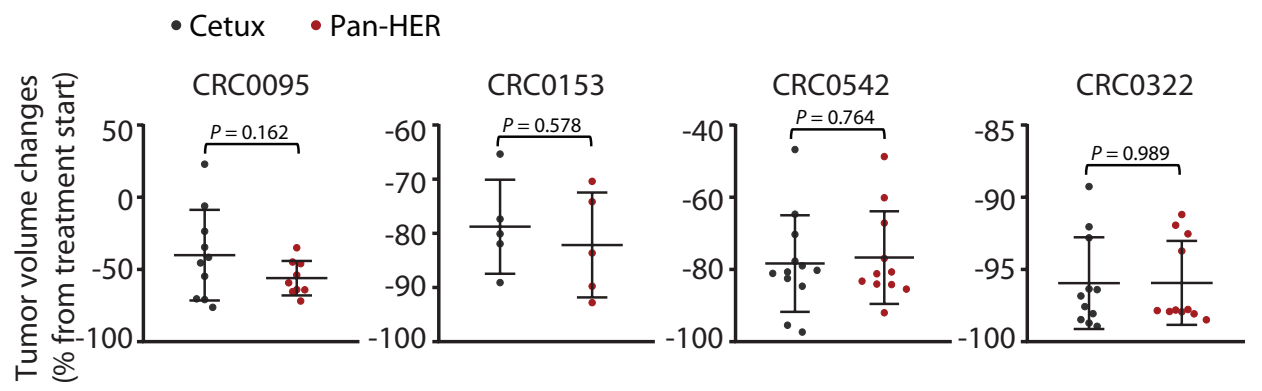

B

- Cetux - Pan-HER

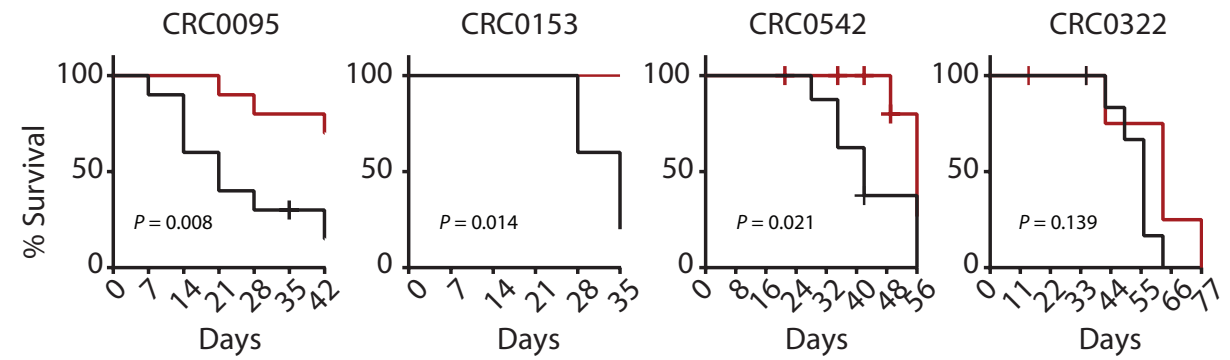

C

D
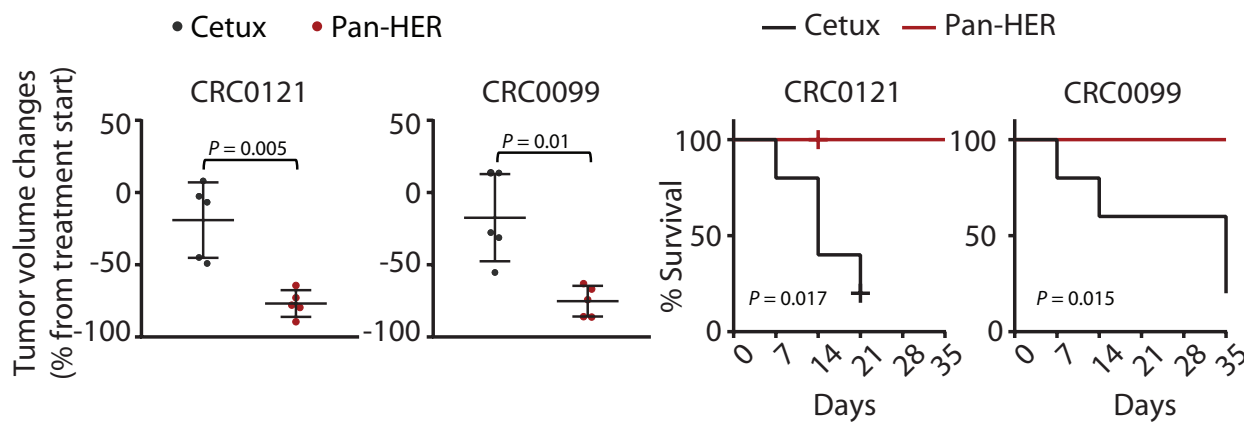

E
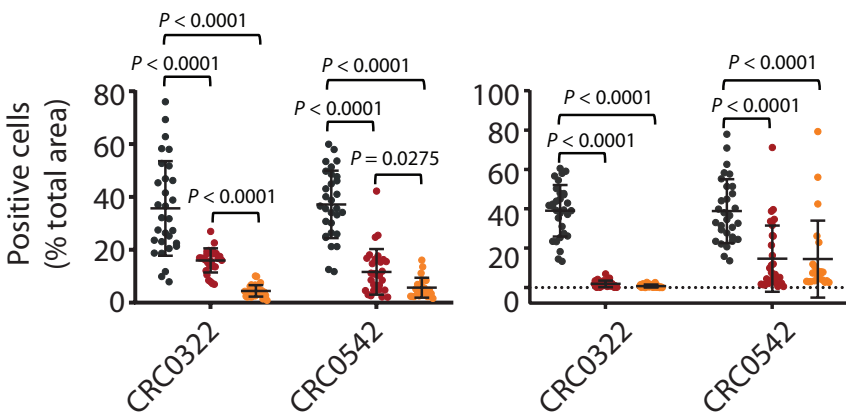

P-S6

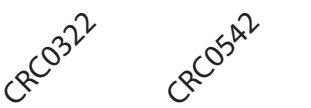

-ERK

- Vehicle

- Cetux

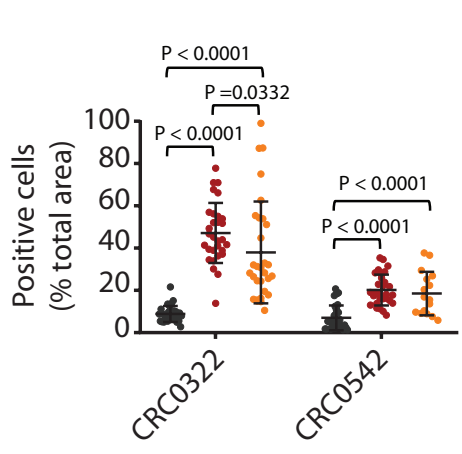

P-HER2

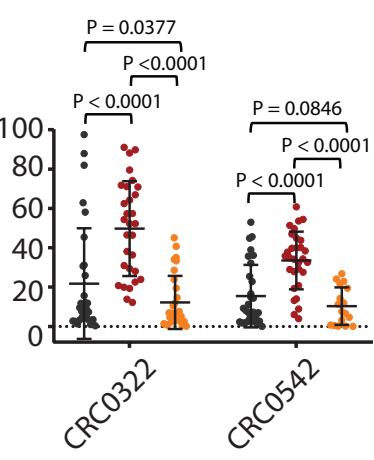

- Pan-HER

P-HER3 


\title{
Colorectal cancer residual disease at maximal response to EGFR blockade displays a druggable Paneth cell-like phenotype
}

\author{
Barbara Lupo, Francesco Sassi, Marika Pinnelli, Francesco Galimi, \\ Eugenia R. Zanella, Valentina Vurchio, Giorgia Migliardi, Paolo Armando Gagliardi, \\ Alberto Puliafito, Daria Manganaro, Paolo Luraghi, Michael Kragh, Mikkel W. Pedersen, \\ Ivan D. Horak, Carla Boccaccio, Enzo Medico, Luca Primo, Daniel Nichol, \\ Inmaculada Spiteri, Timon Heide, Alexandra Vatsiou, Trevor A. Graham, Elena Élez, \\ Guillem Argiles, Paolo Nuciforo, Andrea Sottoriva, Rodrigo Dienstmann, Diego Pasini, \\ Elena Grassi, Claudio Isella, Andrea Bertotti ${ }^{*}$, and Livio Trusolino*
}

*Corresponding authors. E-mails: livio.trusolino@ircc.it; andrea.bertotti@ircc.it

\section{The file includes:}

Materials and Methods

Figure S1. Biological characterization of residual disease after prolonged treatment with cetuximab in representative $\mathrm{mCRC}$ PDXs

Figure S2. Analysis of adaptive changes versus clonal selection induced by cetuximab in representative $\mathrm{mCRC}$ PDXs

Figure S3. ChIP-seq analysis of residual disease after prolonged treatment with cetuximab in representative $\mathrm{mCRC}$ PDXs

Figure S4. Longitudinal analysis of $\beta$-catenin expression at different time points during prolonged treatment with cetuximab in representative mCRC PDXs

Figure S5. GSEA of residual PDXs with signatures of Paneth cells and deep secretory cells in the mouse gut

Figure S6. DEFA5 and $\beta$-catenin double staining in representative mCRC PDXs treated with cetuximab 
Figure S7. Transcript and protein changes of Paneth-cell markers and global gene expression variations in representative $\mathrm{mCRC}$ PDXs during different time points of cetuximab treatment and after therapy suspension

Figure S8. Expression of YAP and YAP targets in representative mCRC PDXs during different time points of cetuximab treatment and after therapy suspension

Figure S9. Inhibition of YAP activity and expression of YAP-dependent genes by cetuximab in CRC cell lines

Figure S10. Expression of secretory/Paneth cell genes after YAP silencing in CRC cell lines

Figure S11. YAP-dependent regulation of Wnt target genes in CRC cell lines

Figure S11. Expression of secretory/Paneth cell genes after YAP silencing in CRC cell lines.

Figure S12. Modulation of YAP transcriptional activity by cetuximab and other inhibitors of the EGFR pathway in CRC cell lines

Figure S13. Expression/activity of doxycycline-inducible YAP-5SA and modulation of secretory/Paneth cell genes by EGFR pathway inhibition in vitro and in vivo

Figure S14. Expression of secretory/Paneth cell genes after YAP silencing or YAP overexpression in vitro and in vivo

Figure S15. Modulation of EGFR family ligands in mCRC PDXs treated with cetuximab

Figure S16. Effects of individual signal inhibition and dual blockade of EGFR and PI3K or EGFR and MEK in CRC cell cultures

Figure S17. Effects of cetuximab on downstream signals in vitro and in vivo

Figure S18. Effects of PI3K inhibitors on downstream signals and tumor growth in vivo

Figure S19. Effects of PI3K inhibition and combined EGFR and PI3K inhibition on mCRC PDX macroscopic residual disease (pre-treatment and end-of-treatment tumor volumes)

Figure S20. Effects of combined EGFR and PI3K inhibition on mCRC PDX microscopic residual disease, apoptosis, and survival 
Figure S21. YAP-dependent transcriptional modulation of HER2 and HER3 in CRC cell lines

Figure S22. Modulation of $H E R 2$ and $H E R 3$ expression in mCRC PDXs during prolonged treatment with cetuximab

Figure S23. DEFA5 and HER2/HER3 double staining in representative mCRC PDXs treated with cetuximab

Figure S24. CRC cell line sensitivity to individual targeting of HER family members

Figure S25. Effects of cetuximab and Pan-HER on EGFR downstream targets in vivo

\section{Other Supplementary Material for this manuscript includes the following:}

Data file S1 (Microsoft Excel format). List of genes subject to mutational and gene copy number analysis

Data file S2 (Microsoft Excel format). GSEA of gene expression changes induced by cetuximab in mCRC PDXs

Data file S3 (Microsoft Excel format). Ingenuity pathway analysis of gene expression changes induced by cetuximab in $\mathrm{mCRC}$ PDXs

Data file S4 (Microsoft Excel format). Expression changes of secretory/Paneth cell genes induced by cetuximab in the reference collection (GSE108277)

Data file S5 (Microsoft Excel format). Original data

Data file S6 (Microsoft Excel format). Taqman probes used for RT-qPCR

\section{SUPPLEMENTARY MATERIALS AND METHODS}

\section{ChIP-seq analysis of PDXs}

Frozen PDX samples were disaggregated in ice-cold PBS in the presence of protease inhibitors. Tumor suspensions were cross-linked first with $2 \mathrm{mM}$ disuccinimidyl glutarate (Covachem) and then with $1 \%$ formaldehyde (Fisher). Chromatin was sonicated in IP Buffer $(100 \mathrm{mmol} / \mathrm{L}$ Tris $\mathrm{pH}$ 
8.6, $0.3 \%$ SDS, $1.7 \%$ Triton $\mathrm{X}-100$, and $5 \mathrm{mmol} / \mathrm{L}$ EDTA) to an average length of $500-1000 \mathrm{bp}$. Samples were immunoprecipitated overnight at $4^{\circ} \mathrm{C}$ with a rabbit anti- $\beta$-catenin antibody (Cell Signaling Technology). Purified DNA was sonicated to an average length of $200 \mathrm{bp}$ and used for library preparation. Sequencing data were analyzed using QARI and PMS modules of the EpiMINE software (56).

\section{Immunohistochemistry and morphometric analyses}

Tumors were formalin-fixed, paraffin-embedded, and subjected to hematoxylin-and-eosin or immunoperoxidase staining with the following antibodies: mouse anti-Ki-67 (Dako); rabbit antiphospho-S6 (Ser235/236), rabbit anti-phospho-ERK (Thr202/Tyr204), rabbit anti-cleaved caspase-3, rabbit anti-pospho-HER2 (Tyr1248), rabbit anti phospho-HER3 (Tyr1289), all from Cell Signaling Technology; mouse anti- $\alpha 5$ defensin (Abcam); mouse anti-YAP (Santa Cruz Biotechnology); mouse anti- $\beta$-catenin (BD Biosciences); rabbit anti- $\beta$-catenin (Cell Signaling). After incubation with secondary antibodies, immunoreactivities were revealed by incubation in $D A B$ chromogen (Dako). Images were captured with the Leica LAS EZ software using a Leica DM LB microscope. Morphometric quantitation was performed by ImageJ software using spectral image segmentation. For double-staining experiments, the VECTOR® VIP Peroxidase Substrate Kit (Vector Laboratories) was employed. Antigens detected by Phospho-HER2, phospho-HER3, and rabbit $\beta$-Catenin antibodies were revealed by rabbit secondary antibody followed by DAB; $\alpha 5$ defensin immunoreactivity was revealed by mouse secondary antibody and VECTOR $\circledast$ VIP purple chromogen. Morphometric quantitation was performed by image $\mathrm{J}$ software using color deconvolution segmentation. Software outputs were manually verified by visual inspection of digital images.

\section{Gene expression analyses}

For RT-qPCR experiments, total RNA was extracted using the Maxwell@ Instrument (Promega) and reverse-transcribed using High-Capacity cDNA reverse transcription (Life Technologies). Results were normalized to the average of two housekeeper genes. The Taqman probes (all provided by ThermoFisher except for ASCL2, which was purchased from Integrated DNA Technologies) are listed in data file S6. For RNA-seq analysis, 1.5 to $2.5 \mu \mathrm{g}$ RNA were sequenced using the TruSeq stranded mRNA kit from Illumina. FastQ files were processed with the Salmon tool, quality-checked with multiQC and normalized for quality assessment. Principal component analysis was then performed on the gene expression profiles. For microarray experiments, RNA was extracted using the miRNeasy Mini Kit (Qiagen). Synthesis of cDNA and biotinylated cRNA was performed using the IlluminaTotalPrep RNA Amplification Kit (Ambion). Quality assessment and quantitation of total RNA and cRNAs were performed with Agilent RNA kits on a Bioanalyzer 2100 (Agilent). Hybridization of cRNAs was carried out using Illumina Human 48k gene chips (Human HT-12 V4 BeadChip). Array washing was performed by lllumina High Temp Wash Buffer for $10^{\prime}$ at $55^{\circ} \mathrm{C}$, followed by staining using streptavidin-Cy3 dyes (Amersham Biosciences). 
Hybridized arrays were stained and scanned in a Beadstation 500 (Illumina). For bioinformatic analyses of microarray data, probe intensity data were extracted using the Illumina Genome Studio software (Genome Studio V2011.1) and subjected to Loess normalization using the Lumi R package. To minimize the noise, probes that generated detectable signals due to cross-species hybridization of transcripts deriving from murine infiltrates in PDX tissues were removed from the analysis. Finally, for each gene the probe displaying the highest signal variance among those that were detected (Genome Studio detection $P=0$ ) was selected for further analyses. GSEA was performed using the dedicated software (software.broadinstitute.org/gsea/index.jsp); the statistical significance of enrichment was estimated based on default settings and 1000 gene permutations. IPA was applied to signatures of genes modulated by cetuximab by at least 2 folds. Enrichment (chi-square $P$ ) was estimated against the "large intestine" and "colon cancer cell lines" background provided by the IPA software. Gene expression microarray data generated in the course of this study have been deposited in the GEO database with accession number GSE108277.

\section{Genomic analyses}

For WES, exome libraries were generated from $200 \mathrm{ng}$ DNA using the Agilent SureSelectXT2 Human All Exon V5 Kit and were sequenced on the Illumina HiSeq2500 platform. Median coverage achieved after duplicates was 45x and 62x for CRC0252 and CRC0542, respectively. Adapter trimming was performed with Skewer v0.1.126 with minimum read length after trimming of 35 and mean quality value before trimming of 10 . Trimmed reads were aligned to the full human reference genome hg19 with Burrows-Wheeler Aligner (BWA) v0.7.12. PCR duplicates were marked using Picard tools. Joint mutation calling between multiple samples from the same patient was performed using Platypus v0.8.1. Previously sequenced normal sample was used as reference for somatic variant calling (20). The following filtering criteria were used to call somatic variants in WES samples: i) only variants with Platypus filter PASS, alleleBias, Q20, QD, SC and HapScore were kept; ii) minimum coverage and genotype quality of 10 was required; iii) variants in segmental duplicated regions and centromeric regions were removed; iv) a minimum of 3 reads covering the variant in at least one of the tumor samples per patient were considered; v) 0 number of reads covering the variant in the germline sample; and vi) genotype $0 / 0$ in the germline sample. Only somatic alterations and indels with a variant allele frequency higher than $5 \%$ were considered.

\section{Cell cultures, reagents, vectors, viral infection, and YAP reporter assays}

$\mathrm{NCl}-\mathrm{HCA} 46$ cells were purchased from Sigma Aldrich and were cultured in DMEM; DiFi cells (from J. Baselga) were cultured in F12. The genetic identity of cell lines was validated by short tandem repeat profiling (Cell ID, Promega). CRC0078 colospheres were maintained as described (57) in the presence of $20 \mathrm{ng} / \mathrm{ml}$ EGF. BTC and doxycycline were from Sigma Aldrich. Targeted agents for in vivo or in vitro studies included: cetuximab (Merck); trastuzumab (Roche); Pan-HER and the isolated HER2 and HER3 components of Pan-HER (Symphogen); selumetinib, dactolisib, alpelisib (Carbosynth); BMS345541, ruxolitinib, PLX4720, everolimus, pictilisib, MK2206, and buparlisib 
(Selleck Chemicals). YAP-5SA (mutation sites: S61A, S109A, S127A, S164A, S381A) was cloned into the PS100069 lentiviral vector (Origene) for constitutive expression and into the Lenti- $X^{\text {TM }}$ TetOne ${ }^{\mathrm{TM}}$ Inducible Expression System-Puro (Takara) for doxycycline-regulated expression. The lentiviral vector expressing Myc-DDK-tagged wild type YAP was provided by Origene. The MISSION YAP-targeting shRNAs, as well as the non-targeting control vector, were from Sigma Aldrich. The sequence of YAP-targeting shRNAs is the following:

YAP_ShRNA-1:

CCGGGCCACCAAGCTAGATAAAGAACTCGAGTTCTTTATCTAGCTTGGTGGCTTTTTG YAP_ShRNA-2:

CCGGCAGGTGATACTATCAACCAAACTCGAGTTTGGTTGATAGTATCACCTGTTTTTG. The TOP-GFP lentiviral vector was a gift from Laurie Ailles (Princess Margaret Cancer Center, University Health Network, Toronto, Canada). Lentiviral vectors were produced by LipofectAMINE 2000 (Invitrogen)-mediated transfection of 293T cells (ATCC): To evaluate YAP activity, cells at 80 $\%$ confluence were transiently transfected with the 8xGTIIC-luc YAP-reporter construct (Addgene) using LipofectAMINE 2000. Luciferase activity was assayed $72 \mathrm{~h}$ after transfection using the Luciferase Assay System (Promega) and a GloMax 96 microplate luminometer (Promega). For determination of $8 \times$ GTIIC-luciferase plasmid concentration, equivalent volumes of DNA were used. DCts were obtained by a $40-\mathrm{Ct}$ transformation, and relative expression levels were expressed as $2^{\wedge} \mathrm{DDCt}$ (median centered).

\section{Biological assays}

On day 0 , cells were plated at clonal density $(20 \mathrm{cells} / \mu \mathrm{l}$ ) in complete medium (for CRC0078, EGF was reduced to $4 \mathrm{ng} / \mathrm{ml}$ ). On day 1 , cells were treated as indicated in the figure legends. On day 2 , apoptotic activity was measured using the caspase-Glo 3/7 luminescent assay kit (Promega). Results were normalized against viable cells, which were plated in parallel, treated with the same modalities, and assessed by ATP content (Cell Titer-Glo, Promega). Viability assays in the presence of BTC, cetuximab, trastuzumab, Pan-HER, or the isolated HER2 and HER3 components of Pan-HER were conducted for $72 \mathrm{~h}$. Cell numbers were quantitated by ATP content. EDU incorporation in vitro was assessed using the Click-iT ${ }^{\text {TM }}$ Plus EdU Cell Proliferation Kit for Imaging, Alexa Fluor ${ }^{\mathrm{TM}} 488$ dye (Life Technologies). Organoids were incubated for 3 hours with $10 \mu \mathrm{M}$ EdU prior to fixation and permeabilization; then, cells were stained with DAPI. EDU incorporation in vivo was examined by intraperitoneal injection $(75 \mu \mathrm{g})$. Mice were sacrificed after $24 \mathrm{~h}$, and then tumors were explanted, formalin-fixed, paraffin-embedded and processed as described in (58). 


\section{Western blot analysis}

Proteins were extracted with cold EB buffer (50 mmol/L Hepes pH 7.4, $150 \mathrm{mmol} / \mathrm{L} \mathrm{NaCl}, 1 \%$ Triton $\mathrm{X}-100,10 \%$ glycerol, $5 \mathrm{mmol} / \mathrm{L}$ EDTA, $5 \mathrm{mmol} / \mathrm{L}$ EGTA) in the presence of phosphatase and protease inhibitors. Lysates were run on precasted SDS-polyacrylamide gels (Invitrogen) and transferred to nitrocellulose membranes using a Trans-Blot Turbo Blotting System (Biorad). Membrane-bound antibodies were detected by the enhanced chemiluminescence system (Promega). Primary antibodies were the following: rabbit anti-phosho-EGFR (Tyr1068) (Abcam); rabbit anti-EGFR, rabbit anti-phospho-HER2 (Tyr1248), rabbit anti-phospho-HER3 (Tyr1289), rabbit anti-phospho-AKT (Ser473), rabbit anti-AKT, rabbit anti-phospho-ERK (Thr202/Tyr204), rabbit anti-ERK, rabbit anti-phospho-YAP (Ser127), rabbit anti-phospho-YAP (Ser397), all from Cell Signaling Technology; mouse anti-vinculin and mouse anti- $\alpha$-tubulin (Sigma-Aldrich); mouse anti-c-Myc Tag, rabbit anti-YAP, and mouse anti-HER2, both from Santa Cruz Biotechnology; mouse anti-HER3 (Millipore). 


\section{SUPPLEMENTARY FIGURES}

A

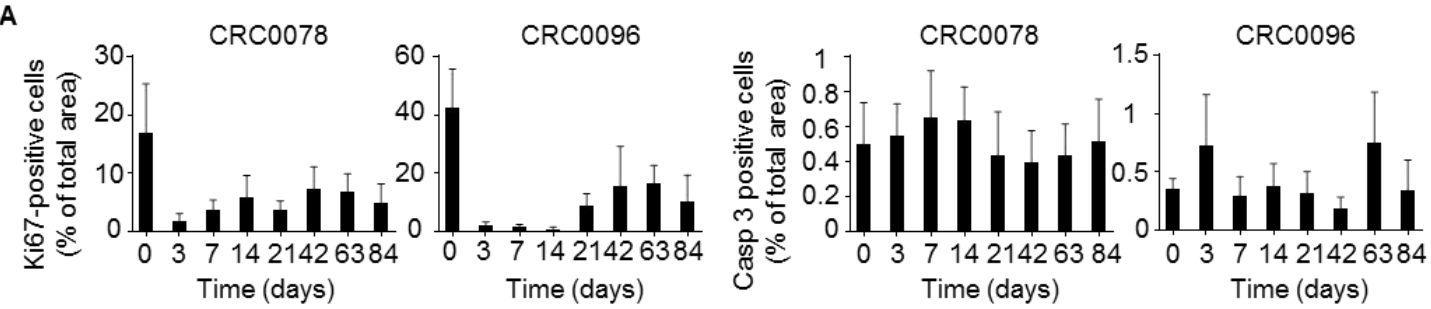

B

PDXs

Organoids
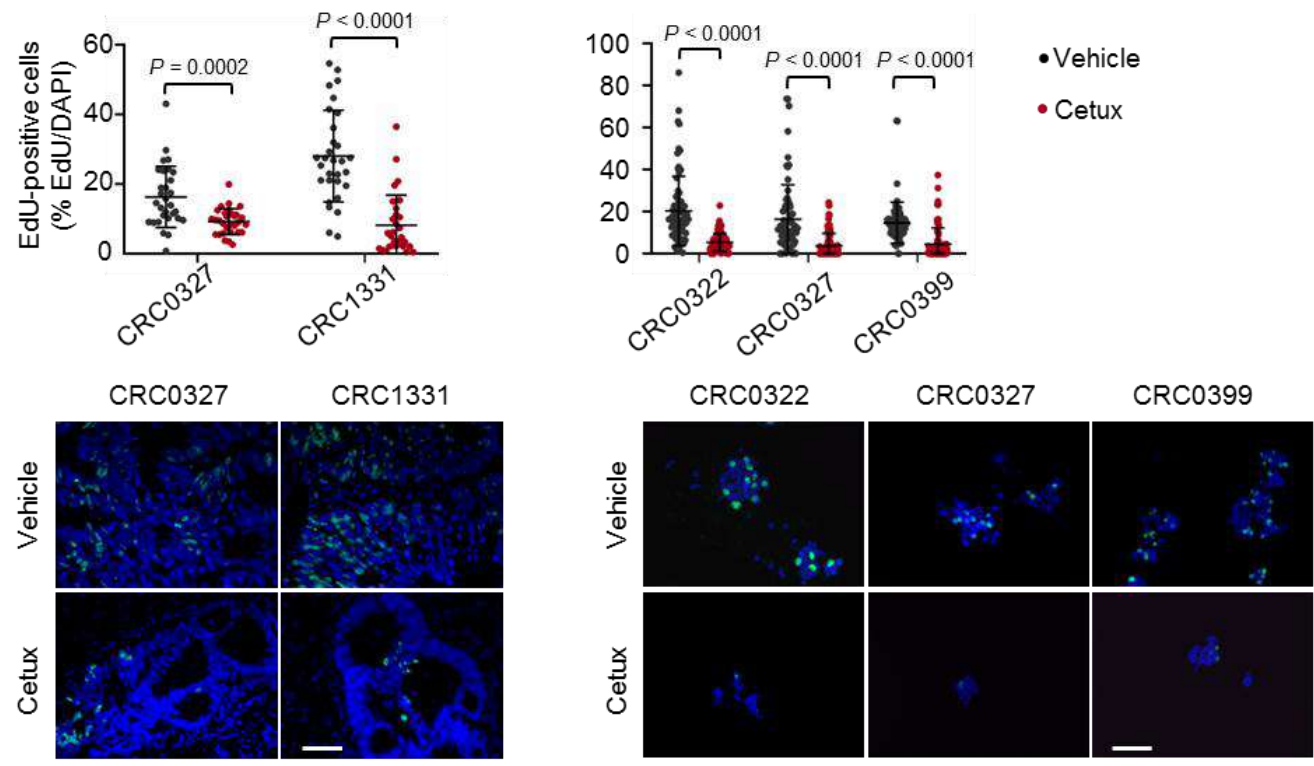

C

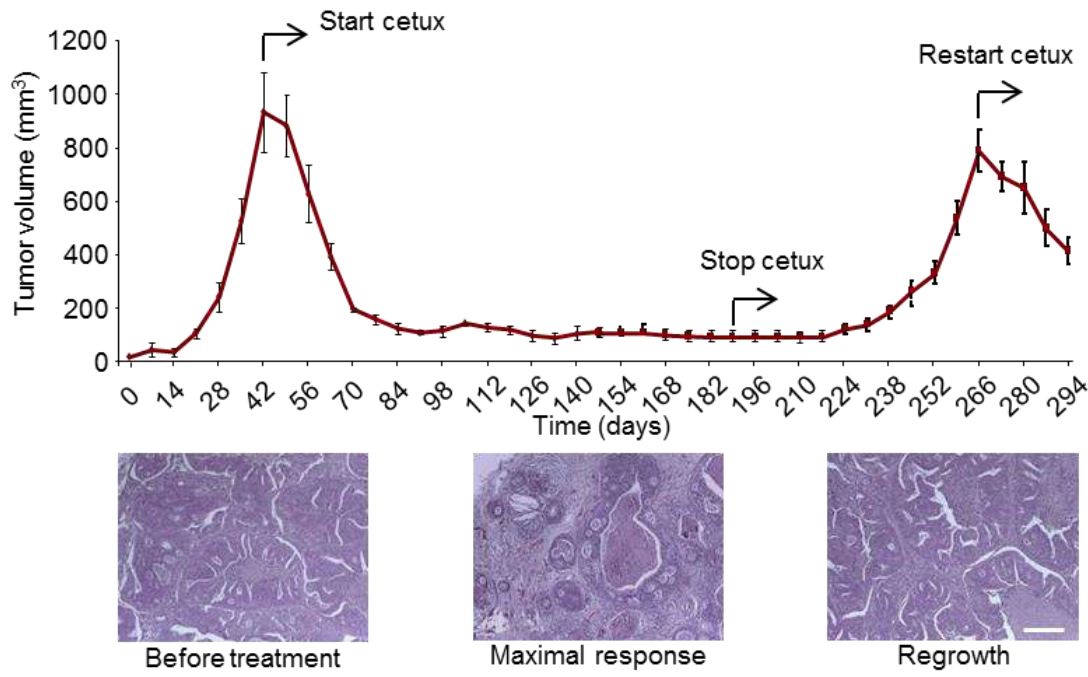


Figure S1. Biological characterization of residual disease after prolonged treatment with cetuximab in representative $\mathrm{mCRC}$ PDXs

(A) Morphometric quantitation of proliferation (left panels) and apoptosis (right panels) in PDX models CRC0078 and CRC0096 treated with cetuximab (20 mg/kg twice a week intraperitoneally) for 12 weeks. At the indicated times, tumors were explanted and subjected to immunohistochemical analysis. Bars are the means \pm SD of 10 optical fields (Ki67, 40X) or 5 optical fields (caspase 3 , 20X) for each time point for each tumor ( $n=20$ to 30 for Ki67 and $n=5$ to 15 for active caspase 3). Casp, caspase. (B) Left panels, morphometric quantitation and representative images of EdU incorporation in two PDXs from the reference collection after treatment with vehicle (until tumors reached an average volume of $1500 \mathrm{~mm}^{3}$ ) or cetuximab (for 6 weeks). At the end of treatment, 3 tumors from 3 different mice were explanted and subjected to immunohistochemical analysis. Each dot represents the value measured in one optical field, with 10 optical fields per tumor $(n=30$ for each condition). Right panels, morphometric quantitation and representative images of EdU incorporation in 3 PDX-derived organoids after treatment with vehicle or cetuximab $(20 \mu \mathrm{g} / \mathrm{ml})$ for 2 weeks. Results are the means \pm SD of 3 biological replicates. Each dot represents the value measured in one optical field, with 25 optical fields per replicate $(n=75)$. Statistical analysis by two-tailed unpaired Welch's $t$-test. Scale bar, $100 \mu \mathrm{m}$ (left panels) or $50 \mu \mathrm{m}$ (right panels). Cetux, cetuximab. (C) Upper panel, tumor growth curve showing the response of PDX model CRC0059 over the course of a 21-week treatment with cetuximab, followed by therapy discontinuation and subsequent drug re-challenging. Values indicate the mean tumor volumes $\pm \operatorname{SEM}(n=6)$. Lower panel, representative hematoxylin-and-eosin images of the same case immediately before treatment, at maximal response ( 6 weeks), and 11 weeks after drug washout. Before treatment the tumor had a well-differentiated phenotype, with cells describing irregular pluristratified tubular/acinar structures with multiple lumens embedded in a scarce stromal matrix. The posttreatment tissue at maximal response displayed reduced cellularity and pseudoglandular remnants scattered among large necrotic areas. After regrowth, the tumor re-acquired the histopathological characteristics of the treatment-naïve counterpart. Scale bar, $500 \mu \mathrm{m}$. 
A

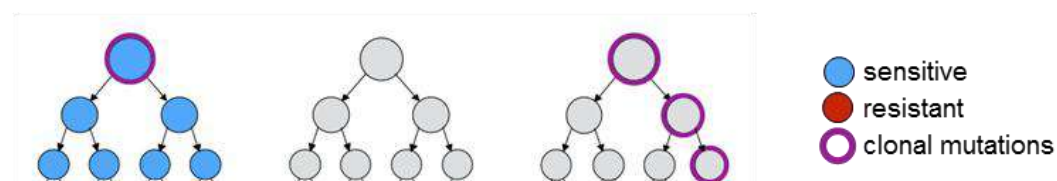

B

Cetux

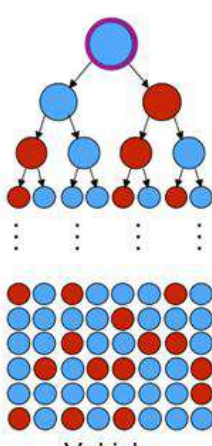

Vehicle

C
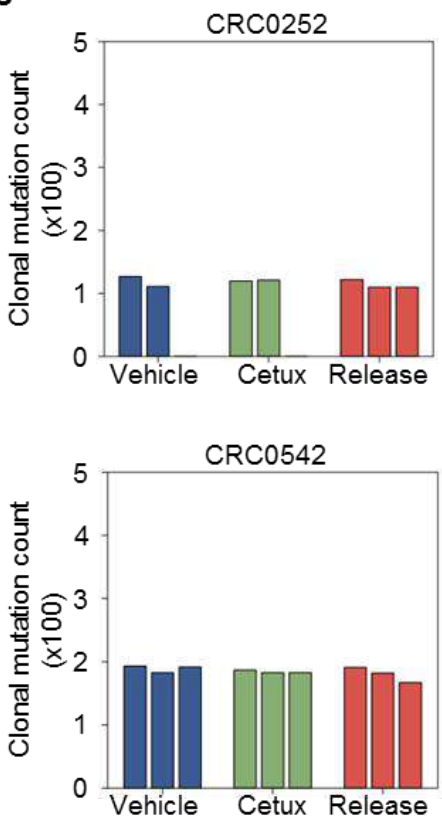

Cetux

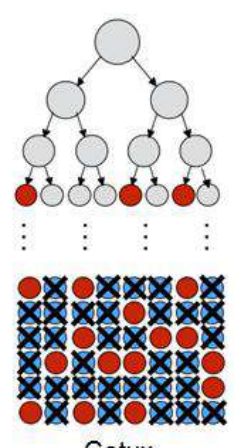

D
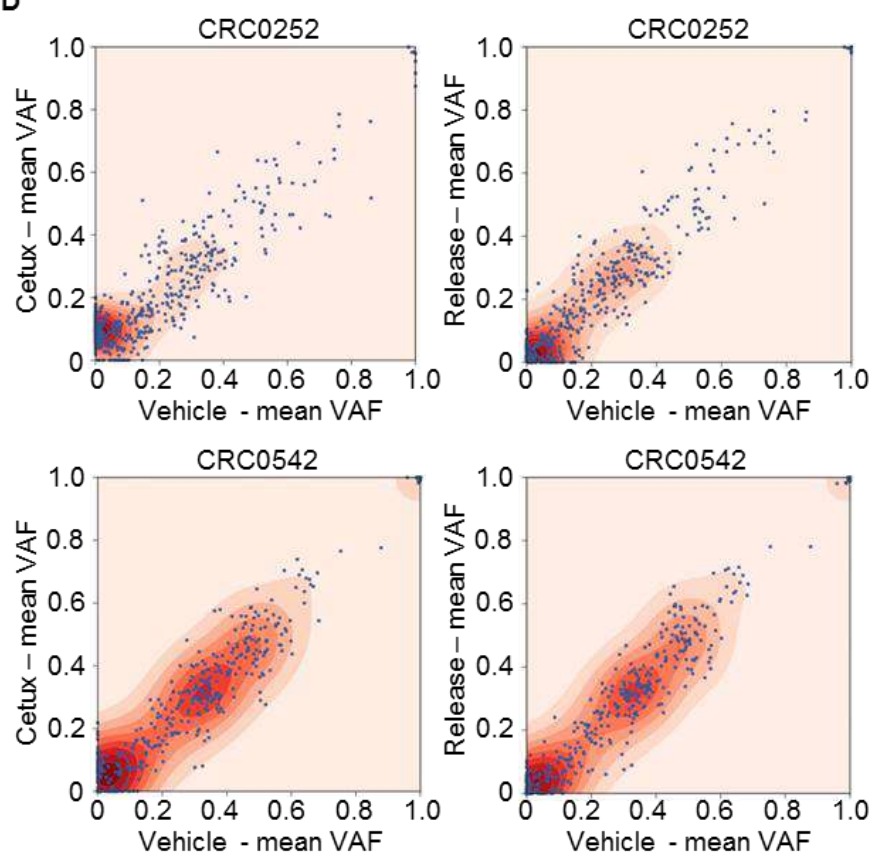

Figure S2. Analysis of adaptive changes versus clonal selection induced by cetuximab in representative mCRC PDXs

(A) Schematic of the changes in clonal mutational load when drug resistance is heritable (e.g. genetically driven). Phylogenetic trees illustrate how selection for a subclonal mutation (subtree, upper right panel) in a heterogeneous population consisting of sensitive (blue dots) and resistant (red dots) cells results in a greater load of clonal/truncal mutations (purple circles) following drug- 
induced selection and after therapy cessation, due to the drug-induced population bottleneck. (B) Schematic of the changes in clonal mutational load when drug resistance is driven by non-heritable or plastic phenotypes. Nodes in the phylogenetic trees are coloured according to a stochastically determined non-heritable phenotype. Surviving cells are an unbiased sub-sample of the population, leaving the number of clonal/truncal mutations unchanged. Drug exposure initially renders the population homogeneous; however, phenotypic heterogeneity returns when therapy ceases. (C) Number of clonal mutations (variant allele frequency greater than or equal to 0.3 ) detected by whole exome sequencing analysis of untreated tumors (Vehicle), cetuximab-treated samples (Cetux), and tumors that had relapsed following drug withdrawal (Release) in PDX models CRC0252 and CRC0542. Bars refer to the number of replicates. (D) Scatter plots showing the mean variant allele frequency of all detected somatic mutations in cetuximab-treated samples and relapsed tumors compared to that detected in untreated tumors. VAF, variant allelic frequency. 


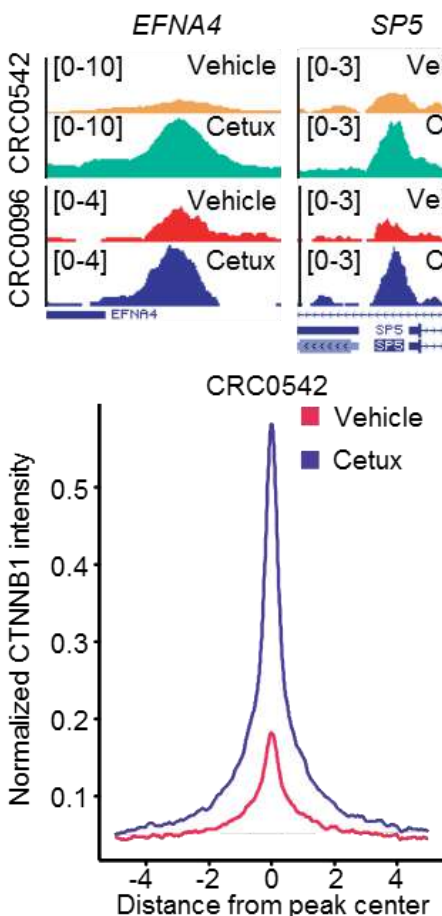

$(\mathrm{Kb})$
MGC32805

[0-5] Vehicle

[0-5] Cetux

[0-3] Vehicle

[0-3] Cetux

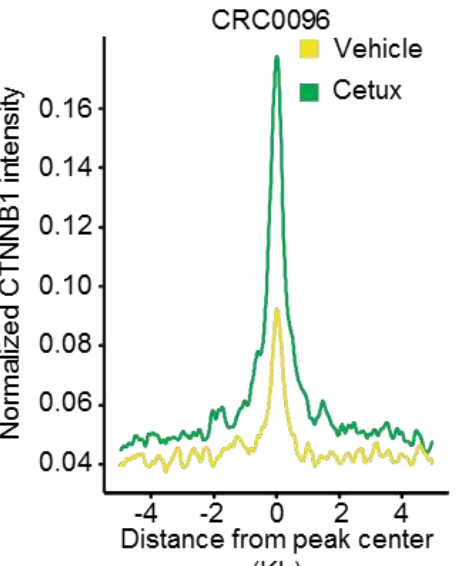

$(\mathrm{Kb})$

Figure S3. ChIP-seq analysis of residual disease after prolonged treatment with cetuximab in representative $\mathrm{mCRC}$ PDXs

Upper panels, genomic snapshots of CTNNB1 ChIP-seq signal at $3 \beta$-catenin target loci from the indicated tumors after treatment with vehicle (until tumors reached an average volume of 1500 $\mathrm{mm}^{3}$ ) or cetuximab (for 6 weeks). Lower panels, cumulative CTNNB1 normalized ChIP-seq intensity from the indicated tumors and conditions at CTNNB1-enriched genomic loci $+/-4 \mathrm{~kb}$ to the peak center. 

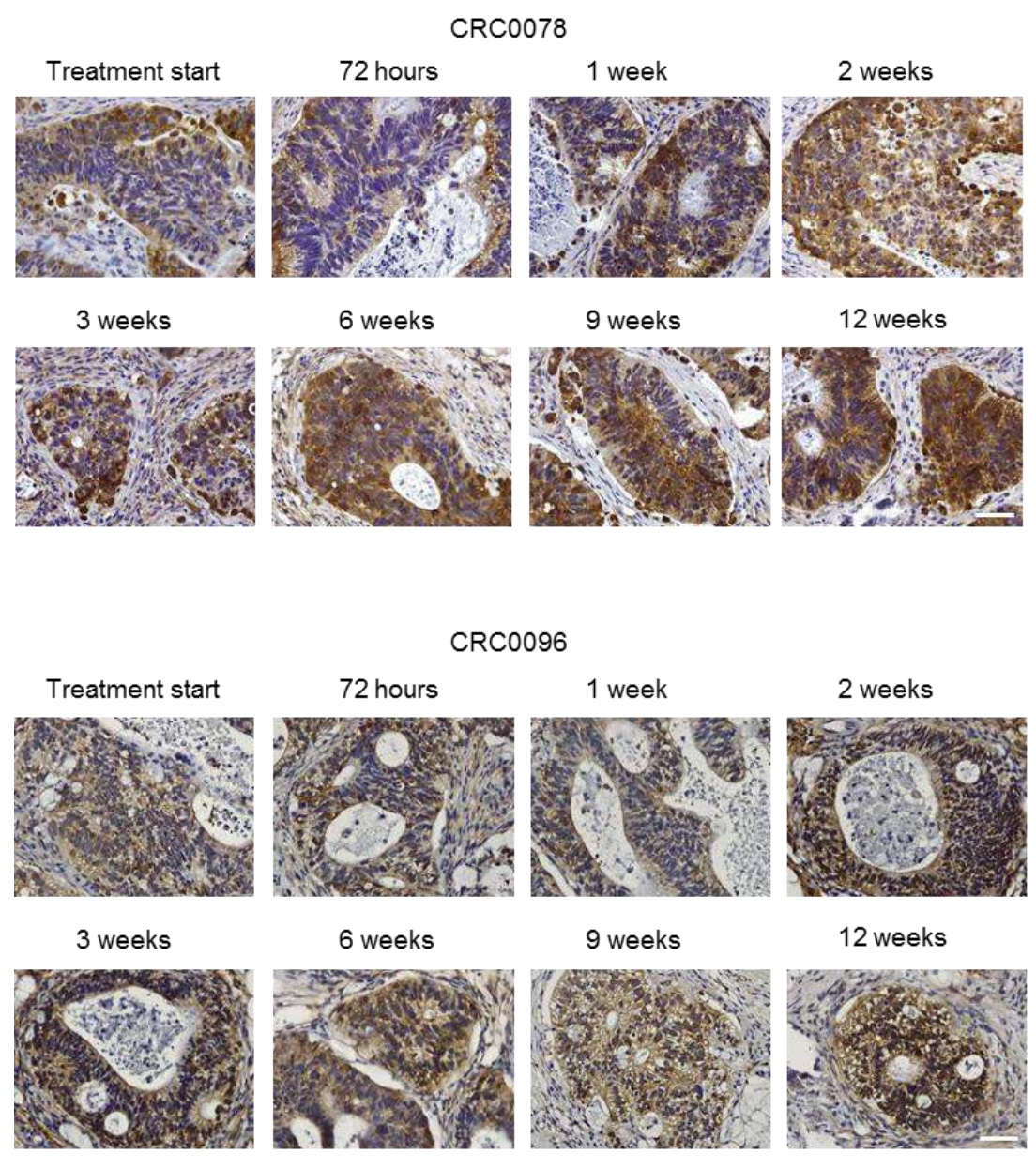

Figure S4. Longitudinal analysis of $\beta$-catenin expression at different time points during prolonged treatment with cetuximab in representative mCRC PDXs

Representative images of $\beta$-catenin expression and localization in two PDX models treated with cetuximab (20 mg/kg twice a week intraperitoneally) and monitored longitudinally for 12 weeks. At the indicated times, tumors were explanted and subjected to immunohistochemical analysis. Scale bar, $50 \mu \mathrm{m}$. 

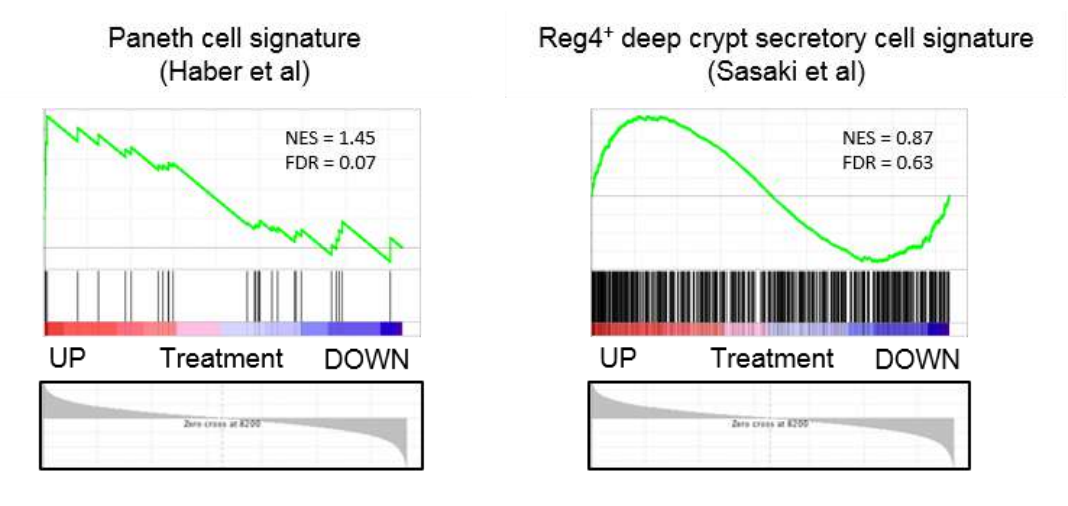

Figure S5. GSEA of residual PDXs with signatures of Paneth cells and deep secretory cells Left panel: GSEA plot showing positive modulation of a Paneth cell signature, obtained in in the mouse small intestinal epithelium (30), in PDXs treated with cetuximab (GSE108277). Right panel: GSEA plot showing no enrichment for a signature of Reg4+ deep crypt secretory cells, which serve as Paneth cell equivalents in the murine colon (31), in PDXs treated with cetuximab. NES, normalized enrichment score; FDR, false discovery rate. 

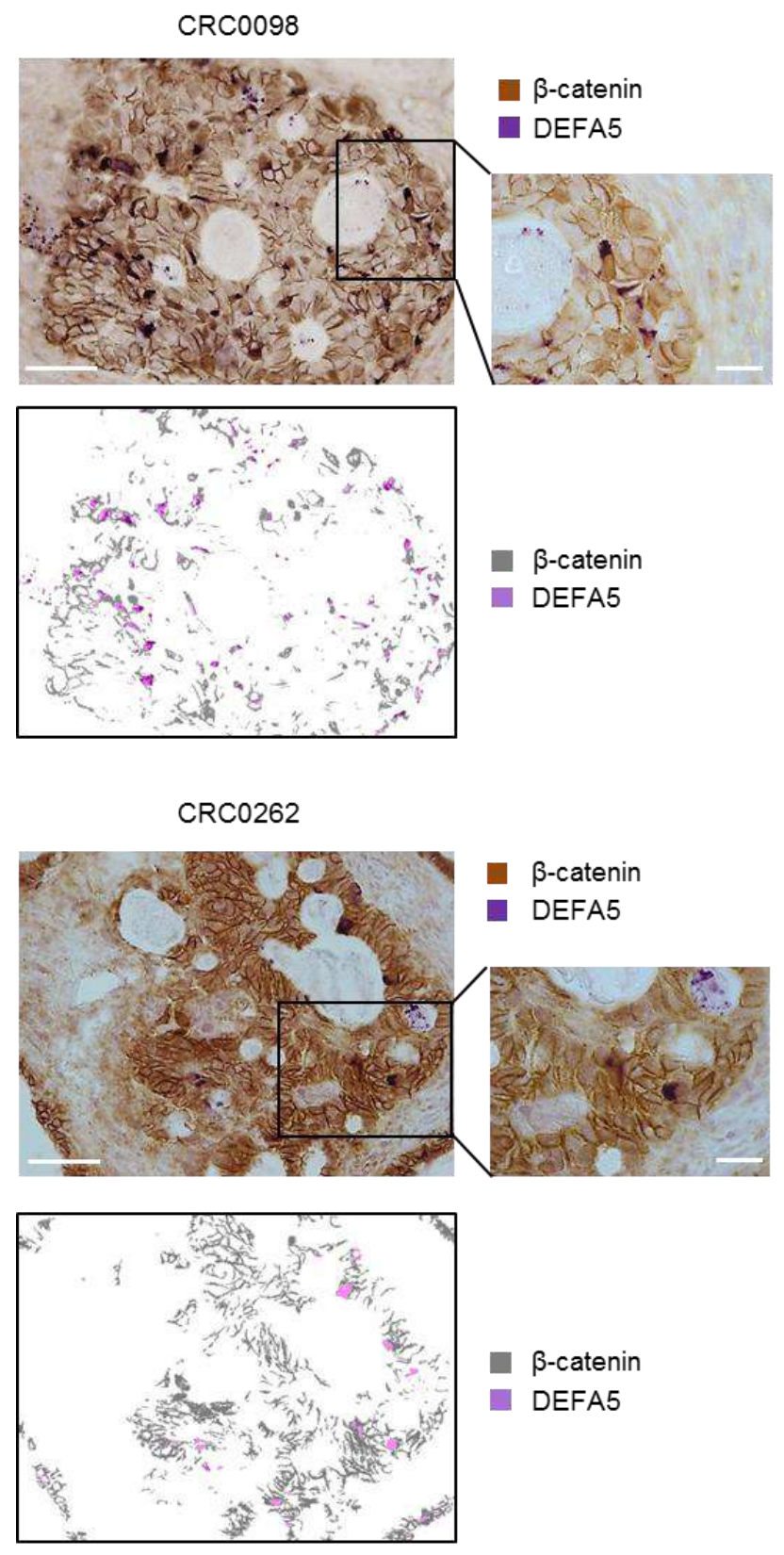

Figure S6. DEFA5 and $\beta$-catenin double staining in representative mCRC PDXs treated with cetuximab

DEFA5 and $\beta$-catenin double staining in 2 PDX models treated with cetuximab $(20 \mathrm{mg} / \mathrm{kg}$ twice a week intraperitoneally) for 6 weeks. For each model, the upper panels are representative images of bright-field optical sections. The lower panels show the corresponding color deconvolution segmentation. Scale bar, $50 \mu \mathrm{M}$ (insets, $20 \mu \mathrm{M}$ ). 


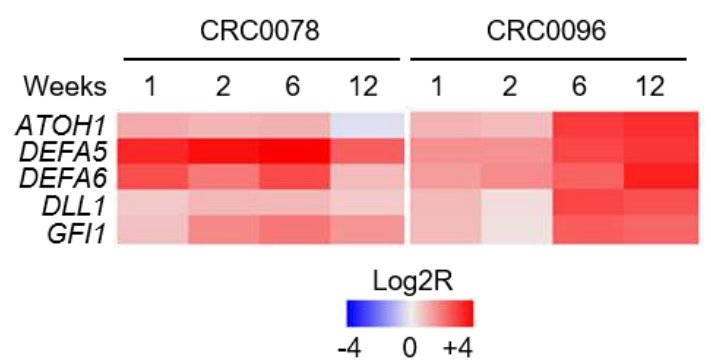

C
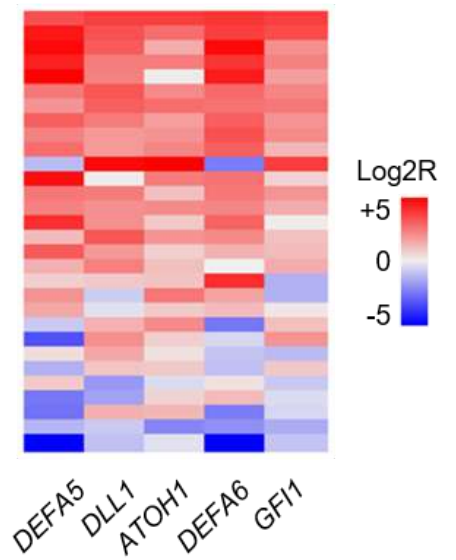

$\mathbf{E}$

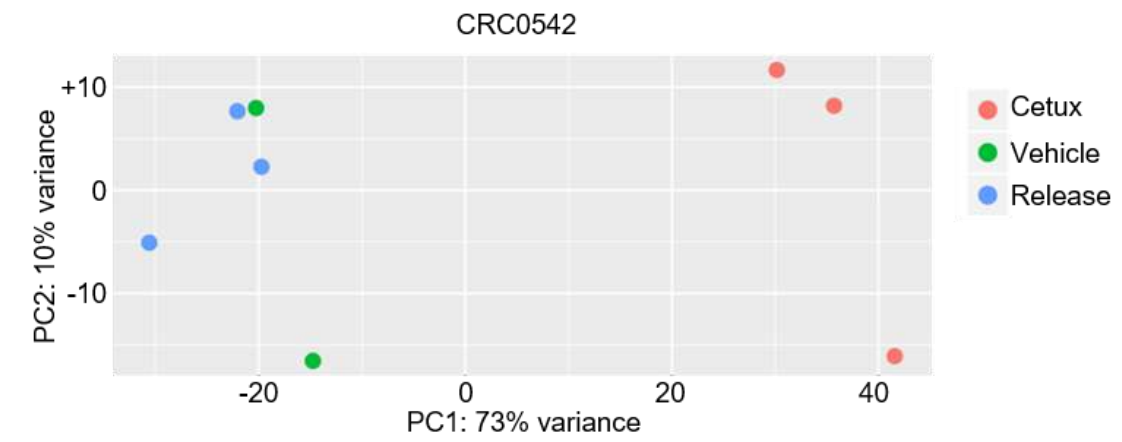

Figure S7. Transcript and protein changes of Paneth-cell markers and global gene expression variations in representative mCRC PDXs during different time points of cetuximab treatment and after therapy suspension

(A) Heatmap showing expression changes for the indicated secretory/Paneth cell markers in 2 representative PDXs treated with cetuximab $(20 \mathrm{mg} / \mathrm{kg}$ twice a week intraperitoneally) and monitored longitudinally for 12 weeks. At the indicated times, 1 tumor from 1 mouse was explanted and subjected to RT-qPCR analysis. Average expression of the 5-gene secretory/Paneth cell signature, Log2R relative to vehicle-treated tumors, for model CRC0078: 1 week, 1.72; 2 weeks, 1.86; 6 weeks, 2.4; 12 weeks, 1.02. Average expression of the 5 -gene secretory/Paneth cell signature, Log2R relative to vehicle-treated tumors, for model CRC0096: 1 week, 1.16; 2 weeks, 
0.92; 6 weeks, 2.68; 12 weeks, 2.94. (B) Morphometric quantitation of DEFA6 protein expression in the 2 PDX models treated with cetuximab and monitored longitudinally for 12 weeks. At the indicated times, tumors were explanted and subjected to immunohistochemical analysis. Bars are the means \pm SD of 5 optical fields (20x) for each tumor ( $n=5$ to 15). (C) Heatmap showing expression changes for the indicated secretory/Paneth-cell markers in PDXs of the reference collection after acute treatment with cetuximab (tumor explant $72 \mathrm{~h}$ after antibody administration), as assessed by RT-qPCR. Average gene expression, Log2R relative to vehicle-treated tumors: DEFA5 1.36, $P<0.0001$; DLL1 1.57, $P<0.0001$; ATOH1 1.45, $P<0.0001$; DEFA6 1.31, $P<$ 0.0001; GFI1 1.01, $P=0.0001$ by two-tailed Wilcoxon test. Benjamini-Hochberg FDR $<0.1$ for all genes. (D) Heatmap showing expression changes for the indicated secretory/Paneth-cell markers in 5 representative PDX models after cetuximab withdrawal, when regrown tumors reached volumes of around $750 \mathrm{~mm}^{3}$. The antibody was discontinued after six weeks of treatment. Gene expression was assessed by RT-qPCR and normalized to cetuximab-treated tumors. Average gene expression of tumors after treatment discontinuation, Log2R relative to cetuximab-treated tumors: DEFA5 -8.75, $P<0.0111 ;$ DEFA6 -7.24, $P=0.007$; DLL1 -3.01, $P=0.0283$; GFI1 -2.31, $P=0.0037 ; A T O H 1-1.54, P=0.2143$ by two-tailed paired Student's t-test. Benjamini-Hochberg FDR $<0.1$ for all genes except $A T O H 1$. (E) Scatter plot showing the two principal components of transcriptional variance (based on RNAseq analysis) in cetuximab-treated samples (Cetux, red dots), relapsed tumors after therapy discontinuation (Release, blue dots) and untreated tumors (Vehicle, green dots) from PDX model CRC0542. Dots refer to the number of replicates. 
A

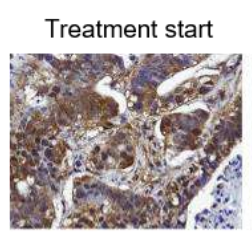

3 weeks
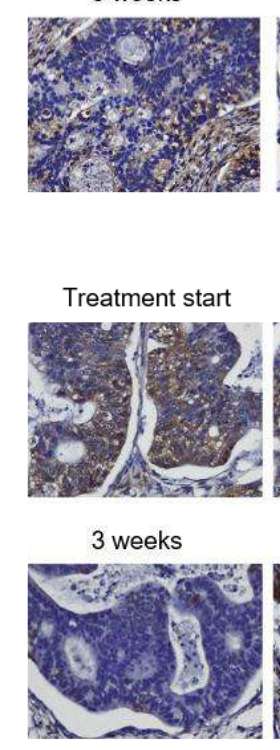

\begin{abstract}
6 weeks
\end{abstract}
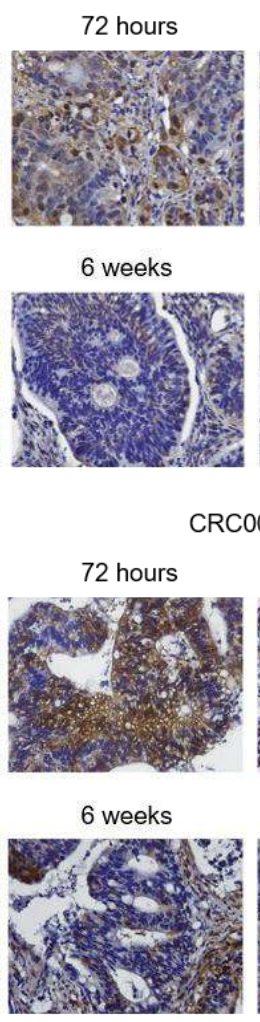

6 weeks
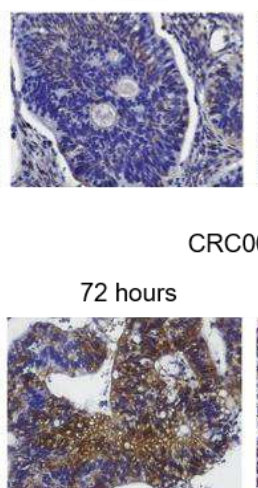

CRC0078

CRC0096

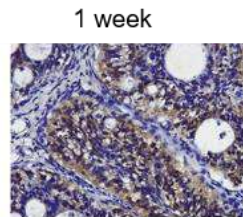

9 weeks
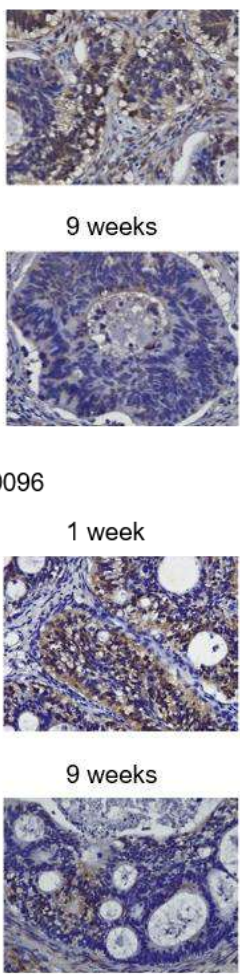

9 weeks
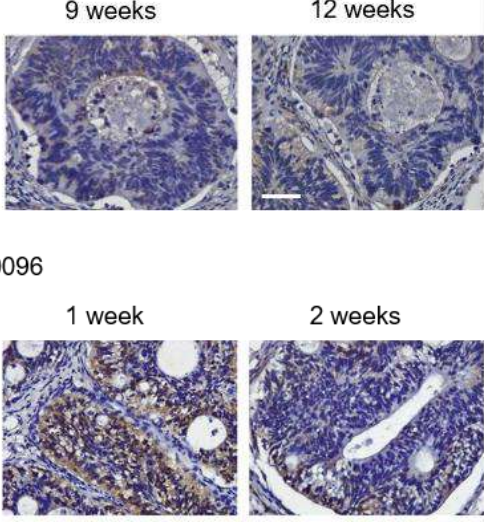

12 weeks

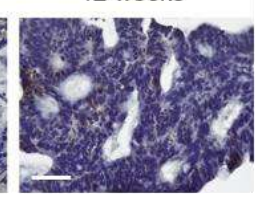

2 weeks

B
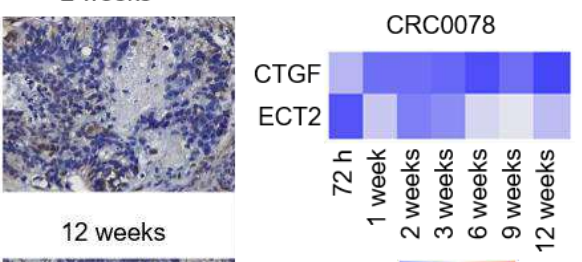

Log2R

$-1.50+1.5$

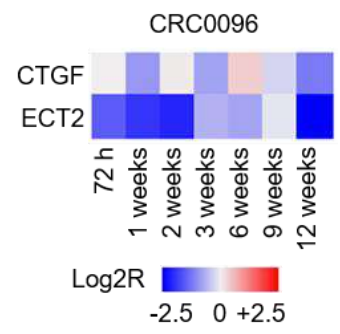

c
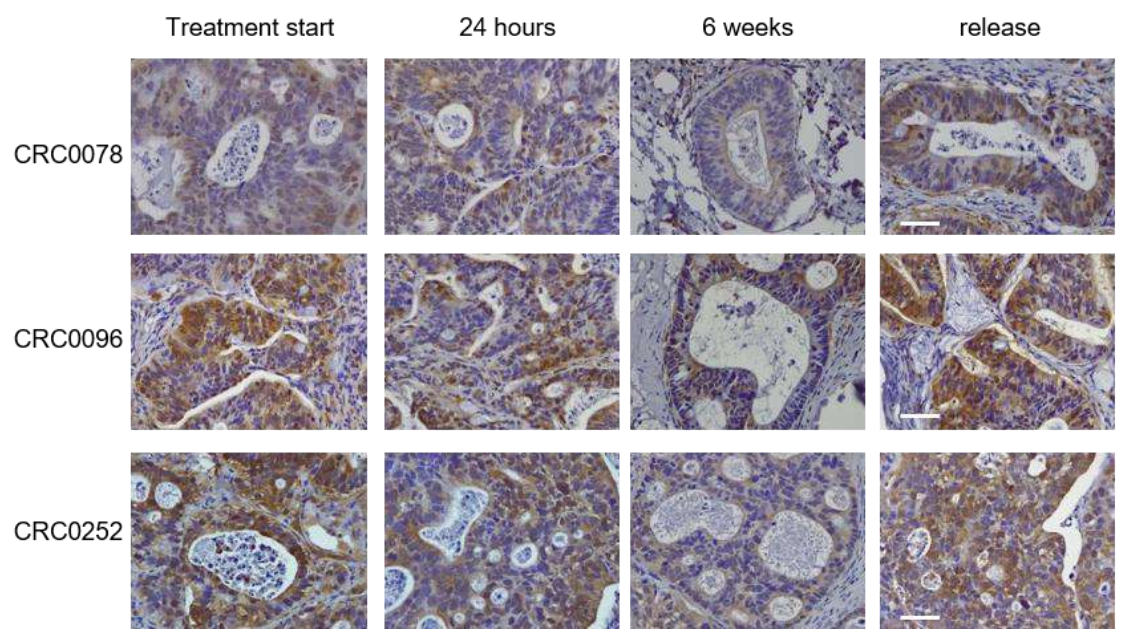

Figure S8. Expression of YAP and YAP targets in representative mCRC PDXs during different time points of cetuximab treatment and after therapy suspension

(A) Representative images of YAP expression and localization in 2 PDX models treated with cetuximab (20 mg/kg twice a week intraperitoneally) and monitored longitudinally for 12 weeks. At the indicated times, tumors were explanted and subjected to immunohistochemical analysis. Scale bar, $50 \mu \mathrm{m}$. (B) Heatmap showing expression changes (assessed by RT-qPCR analysis) for the indicated YAP targets after treatment with cetuximab (20 mg/kg twice a week intraperitoneally) in 
the 2 models shown in panel A. At the indicated times, 1 tumor from 1 mouse was explanted and subjected to RT-qPCR analysis. (C) Representative images of YAP expression and localization in 3 PDX models after treatment with cetuximab for 24 hours and 6 weeks and after antibody withdrawal, when regrown tumors reached volumes of around $750 \mathrm{~mm}^{3}$. Scale bar, $50 \mu \mathrm{m}$. 
A

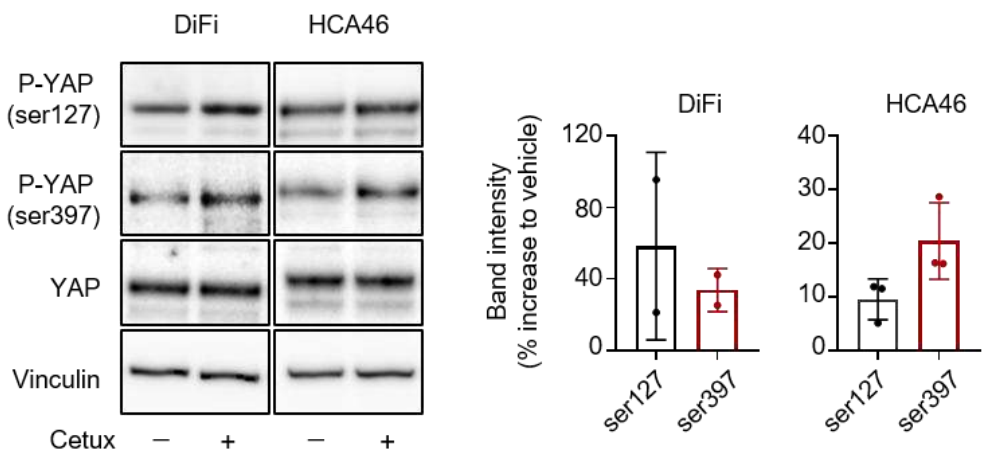

B
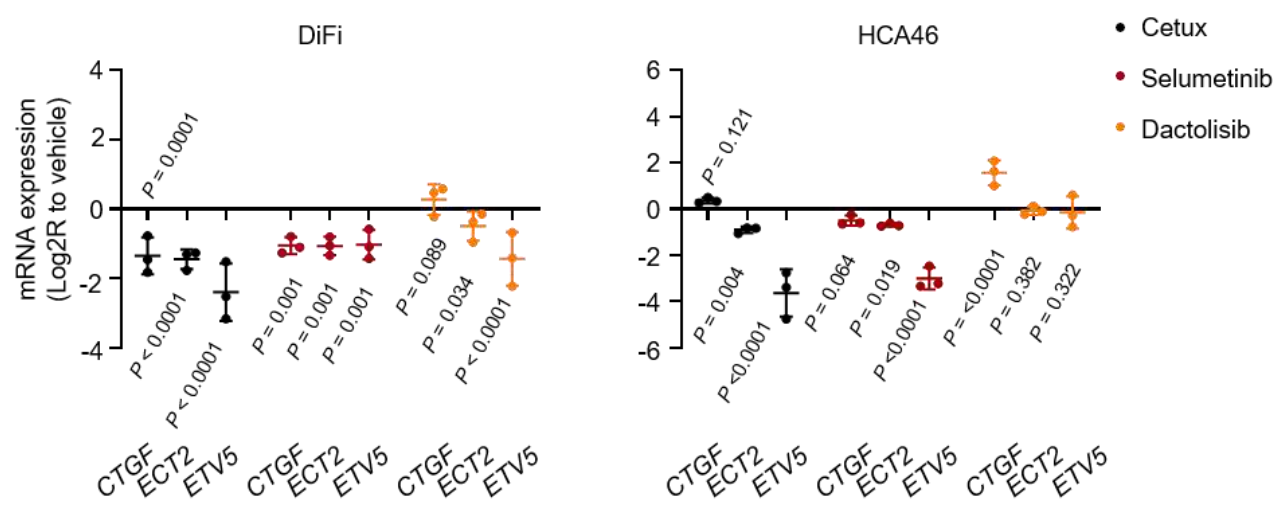

Figure S9. Inhibition of YAP activity and expression of YAP-dependent genes by cetuximab in CRC cell lines

(A) Western blot analysis (left) and densitometric quantitation (right) of YAP phosphorylation in DiFi and HCA46 cell lines treated for 1 hour with $20 \mu \mathrm{g} / \mathrm{ml}$ cetuximab. Total YAP was used for normalization; vinculin was used as a loading control. Western blots for total YAP protein were run with the same lysates as those used for anti-phosphoprotein detection but on different gels. The images shown are representative of 2 (DiFi) or 3 (HCA46) experiments on independent biological replicates. The plots of densitometric analysis show means \pm range (DiFi) or SD (HCA46), with values normalized against vinculin. P-YAP, phospho-YAP; ser127, serine 127; ser397, serine 397; Cetux, cetuximab. (B) RT-qPCR analysis of the indicated YAP target genes in DiFi and HCA46 cells treated for $72 \mathrm{~h}$ with cetuximab $(20 \mu \mathrm{g} / \mathrm{ml})$, selumetinib $(1 \mu \mathrm{M})$, or dactolisib $(250 \mathrm{nM})$. Three independent experiments were performed in technical triplicates. The plots show means \pm SD. Statistical analysis by one-way ANOVA followed by Benjamini, Krieger and Yekutieli FDR correction. 

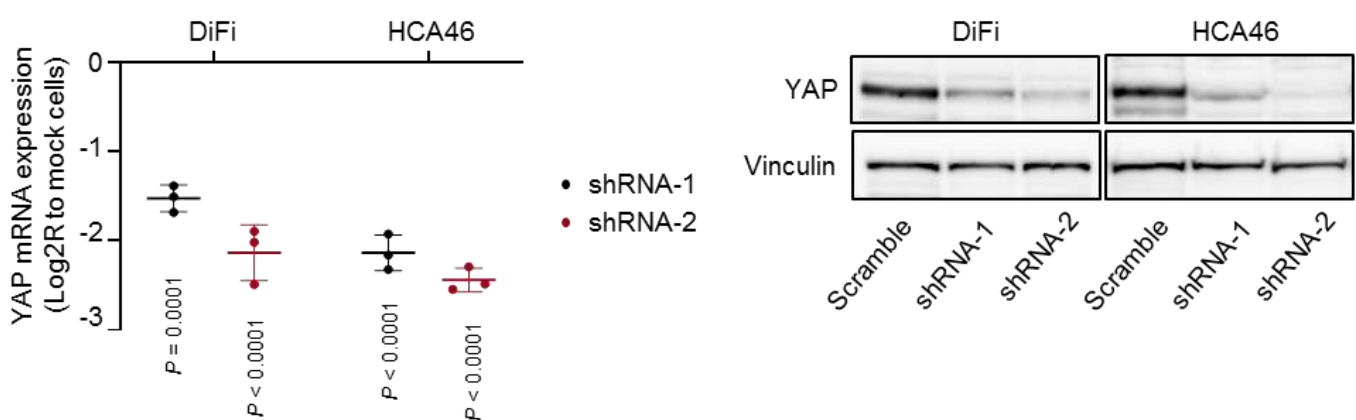

B

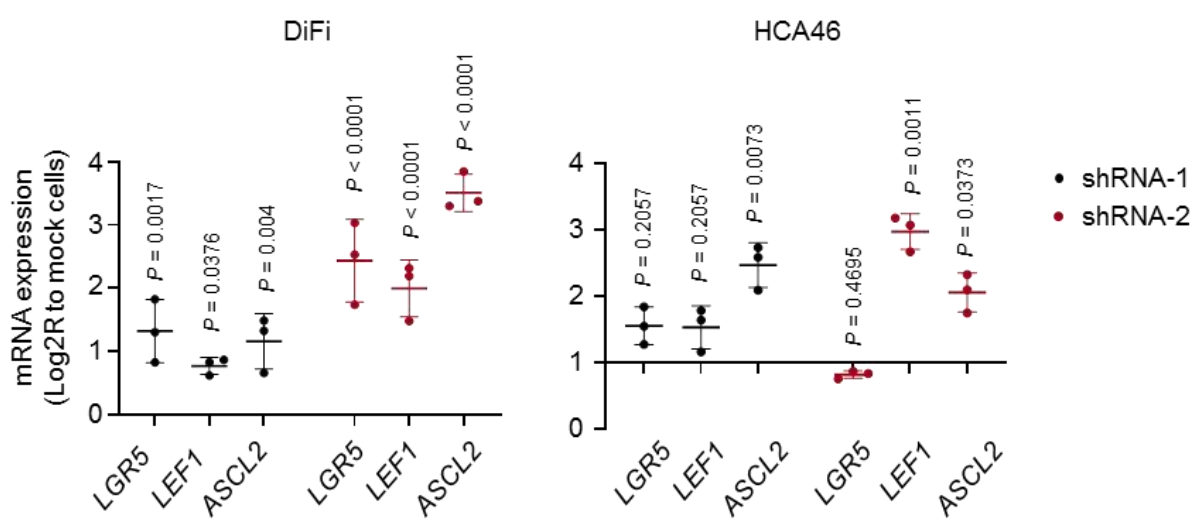

Figure S10. Expression of secretory/Paneth cell genes after YAP silencing in CRC cell lines (A) Transcript (left panel) and protein (right panels) expression of YAP in DiFi and HCA46 cells transduced with two different shRNA lentiviral vectors targeting YAP. For RT-qPCR, 3 independent experiments were performed in biological triplicates $(n=3)$. In western blots, vinculin was used as a loading control. Western blot images are representative of 2 experiments on independent biological replicates. (B) RT-qPCR analysis of Wnt target gene transcripts in DiFi and HCA46 cells after shRNA-based YAP silencing, compared with mock cells. Three independent experiments were performed in technical triplicates. The plots show means \pm SD. Statistical analysis by oneway ANOVA followed by Benjamini, Krieger and Yekutieli FDR correction. 
A

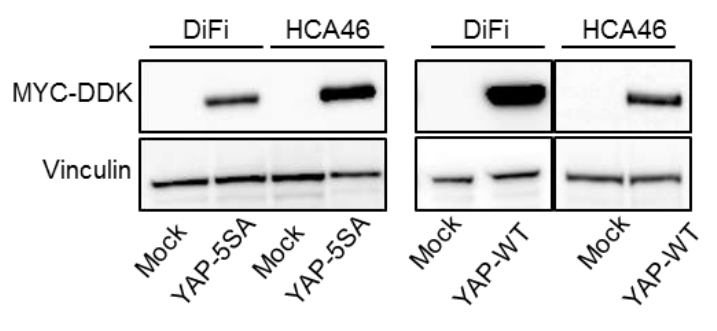

B

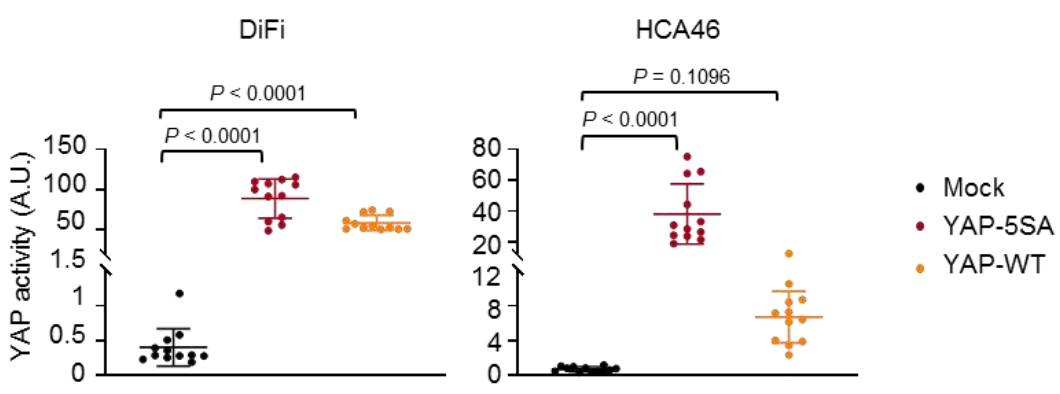

c

DiFi

HCA46

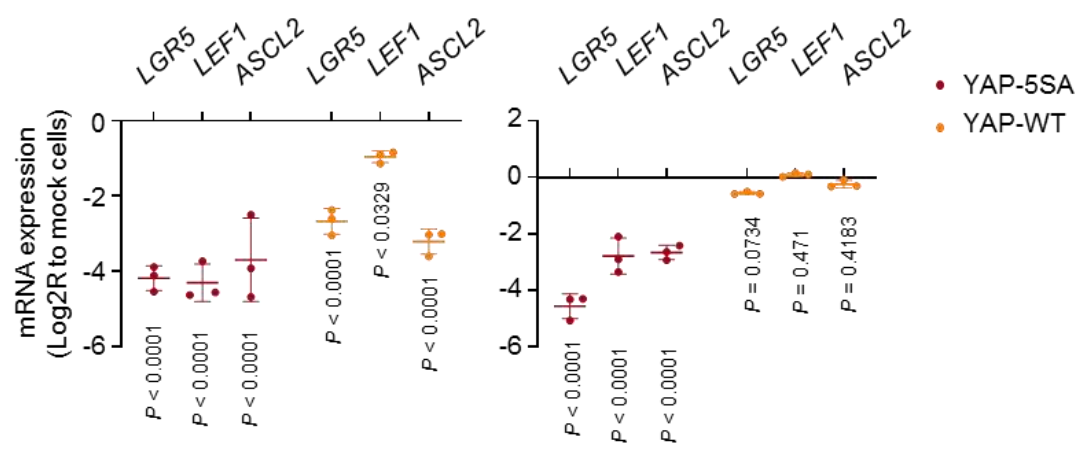

Figure S11. YAP-dependent regulation of Wnt target genes in CRC cell lines

(A) Western blot analysis of total protein extracts from DiFi and HCA46 cells transduced with the control pLVX-IRES-Puro vector (mock), a lentiviral vector enabling constitutive expression of the Myc-DDK-tagged mutant YAP variant YAP-S5A (left panels), or a lentiviral vector encoding MycDDK-tagged wild-type YAP (YAP-WT) (right panels). YAP-S5A and YAP-WT were detected using a Myc antibody. Vinculin was probed as a loading control. (B) Luciferase-based measurement of YAP reporter activity in DiFi and HCA46 cells stably transduced with constitutive YAP-5SA or wildtype YAP and transiently transfected for $72 \mathrm{~h}$ with the 8xGTIIC-luc YAP-reporter construct. Luciferase activity was normalized against reporter plasmid concentration as determined by DNA qPCR and expressed as arbitrary units (A.U.). Three independent experiments were performed in biological quadruplicates $(n=12)$. The plots show means \pm SD. Statistical analysis by one-way ANOVA followed by Benjamini, Krieger and Yekutieli FDR correction. (C) RT-qPCR analysis of the indicated Wnt target genes in DiFi and HCA46 cell lines transduced with YAP-5SA or YAP-WT. 
Three independent experiments were performed in technical triplicates. The plots show means \pm SD. Statistical analysis by one-way ANOVA followed by Benjamini, Krieger and Yekutieli FDR correction.
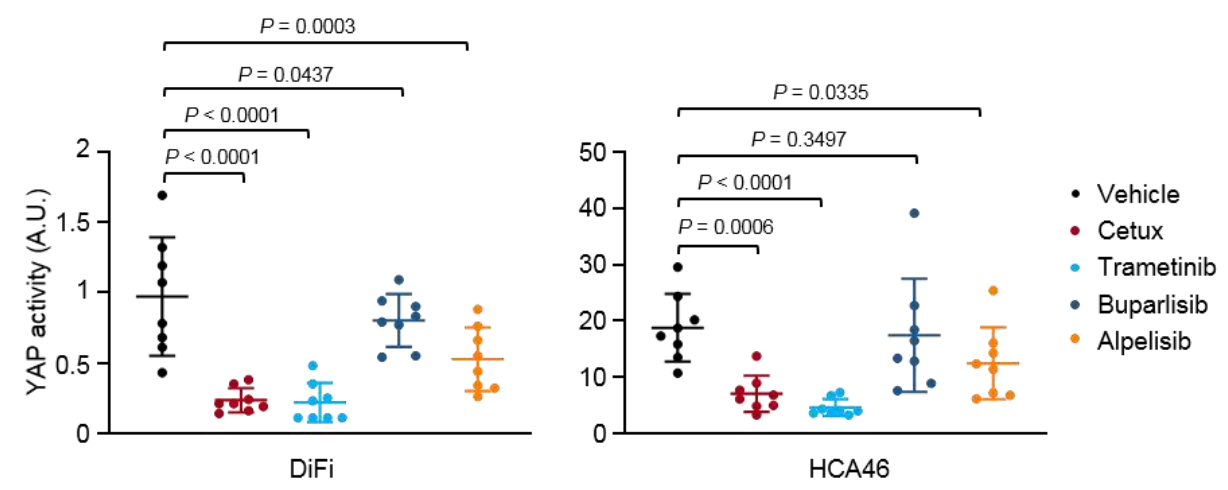

Figure S12. Modulation of YAP transcriptional activity by cetuximab and other inhibitors of the EGFR pathway in CRC cell lines

Measurement of YAP transcriptional activity in DiFi and HCA46 cell lines after treatment for $48 \mathrm{~h}$ with vehicle (DMSO), cetuximab $(20 \mu \mathrm{g} / \mathrm{ml})$, trametinib $(100 \mathrm{nM})$, buparlisib $(0.5 \mu \mathrm{M})$, or alpelisib $(1 \mu \mathrm{M})$. Luciferase activity was normalized against reporter plasmid concentration as determined by DNA qPCR and expressed as arbitrary units (A.U.). Two independent experiments were performed in biological quadruplicates $(n=8)$. The plots show means \pm SD. Statistical analysis by one-way ANOVA followed by Benjamini, Krieger and Yekutieli FDR correction. Cetux, cetuximab. 
A

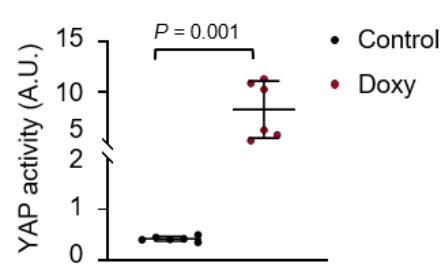

c

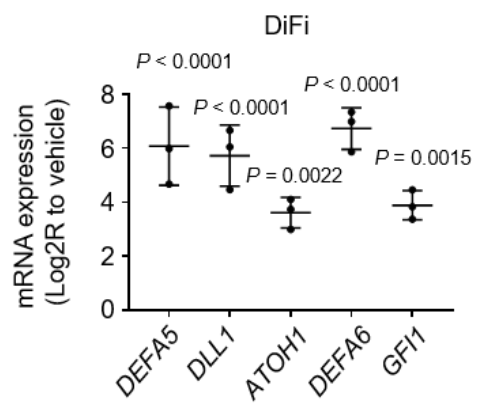

D
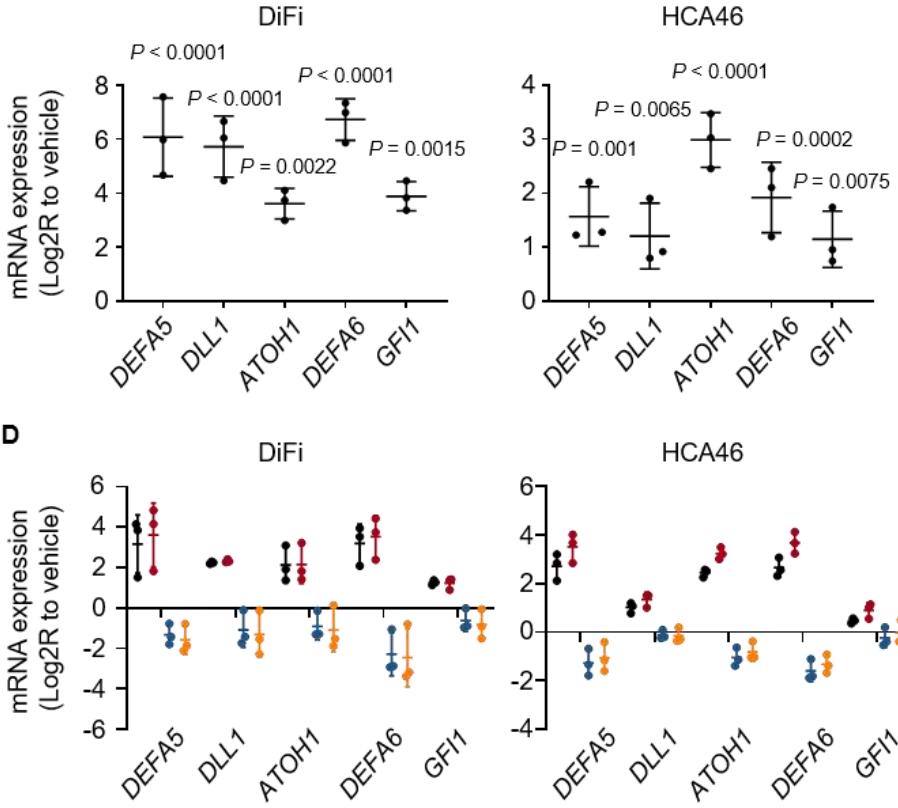

HCA46

HCA46
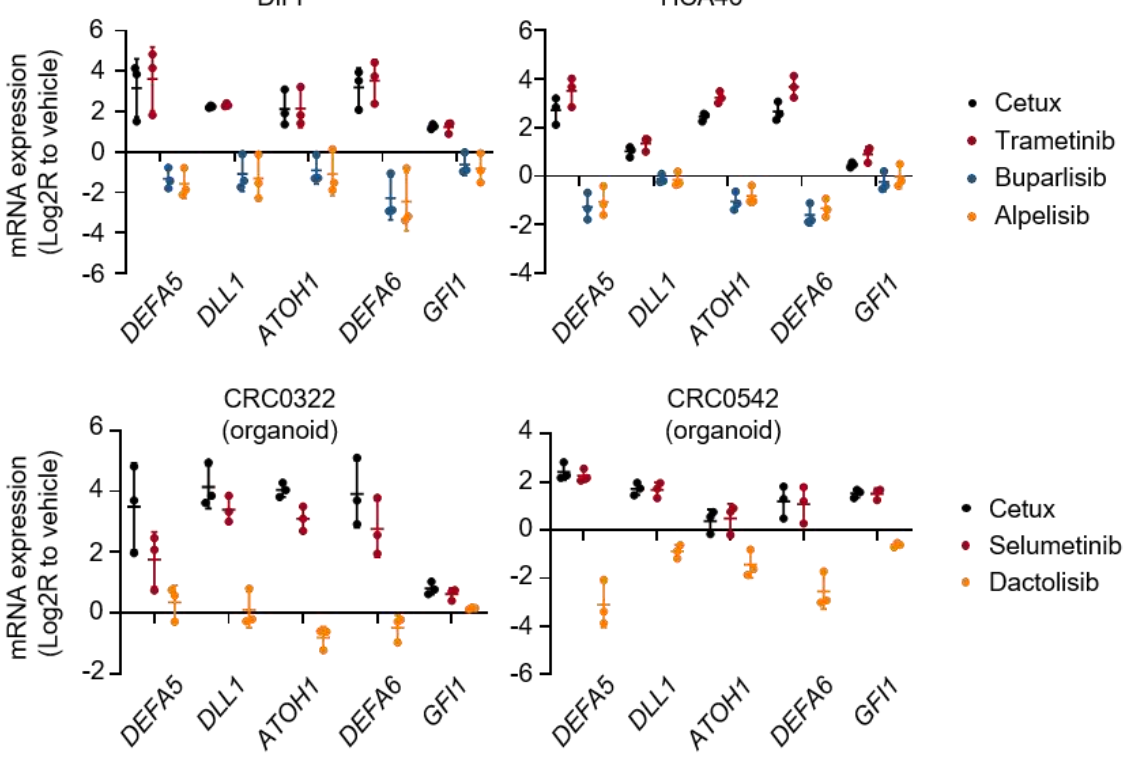

E

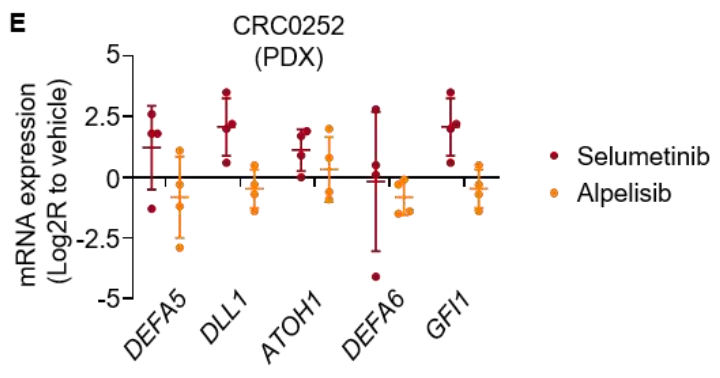

Buparlisib

Alpelisib

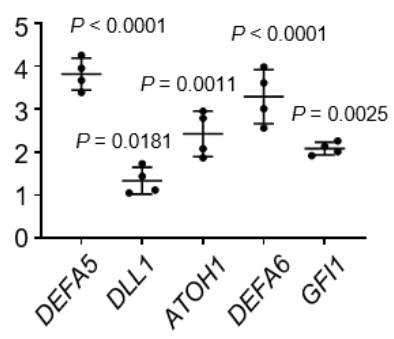

CRC0078

(colosphere) 
transfected for $72 \mathrm{~h}$ with the 8xGTIIC-luc YAP-reporter construct, and either left untreated (control) or treated with doxycycline for $48 \mathrm{~h}$. Luciferase activity was normalized against reporter plasmid concentration as determined by DNA qPCR and expressed as arbitrary units (A.U.). Two independent experiments were performed in biological triplicates $(n=6)$. The plots show means \pm SD. Statistical analysis by two-tailed unpaired Welch's t-test. Doxy, doxycycline. (B) Representative images of YAP expression in DiFi xenografts after treatment with doxycycline (50 $\mathrm{mg} / \mathrm{kg}$ daily oral gavage) for 1 week, compared with vehicle-treated (control) counterparts. Scale bar, $100 \mu \mathrm{m}$ (200 $\mu \mathrm{m}$ for insets). (C) RT-qPCR analysis of the indicated Paneth-cell transcripts in CRC cell lines ( $\mathrm{DiFi}$ and HCA46) and colospheres (CRC0078) treated with $20 \mu \mathrm{g} / \mathrm{ml}$ cetuximab for 72h. Three (DiFi, HCA46) or 4 (CRC0078) independent experiments were performed in technical triplicates. The plots show means \pm SD. Statistical analysis by matched one-way ANOVA followed by Benjamini, Krieger and Yekutieli FDR correction. (D) RT-qPCR analysis of Paneth-cell transcripts in cell lines and organoids treated with the indicated inhibitors for 72h (cetuximab, 20 $\mu \mathrm{g} / \mathrm{ml}$; selumetinib, $1 \mu \mathrm{M}$; trametinib, $100 \mathrm{nM}$; dactolisib, $250 \mathrm{nM}$; buparlisib, $0.5 \mu \mathrm{M}$; alpelisib, 1 $\mu M)$. Three independent experiments were performed in technical triplicates. The effects of EGFR and MEK blockade were positively correlated (DiFi, cetuximab versus trametinib, $r=0.997$; HCA46, cetuximab versus trametinib, $r=0.995$; CRC0322, cetuximab versus selumetinib, $r=0.997$; CRC0542, cetuximab versus selumetinib, $r=0.997$, Pearson's correlation coefficient). In contrast, transcriptional changes induced by PI3K pathway inhibition negatively correlated with those triggered by cetuximab ( $\mathrm{DiFi}$, cetuximab versus buparlisib, $r=-0.832$; cetuximab versus alpelisib, $r=-0.855$; HCA46, cetuximab versus buparlisib, $r=-0.935$; cetuximab versus alpelisib, $r=-0.96$; CRC0322, cetuximab versus dactolisib, $r=-0.415$; CRC0542, cetuximab versus dactolisib, $r=-$ 0.356). Cetux, cetuximab. (E) RT-qPCR analysis of Paneth-cell transcripts in a PDX model treated for 3 weeks with the indicated inhibitors. Four samples for each condition were analyzed in technical triplicates. The plots show means \pm SD. 
A
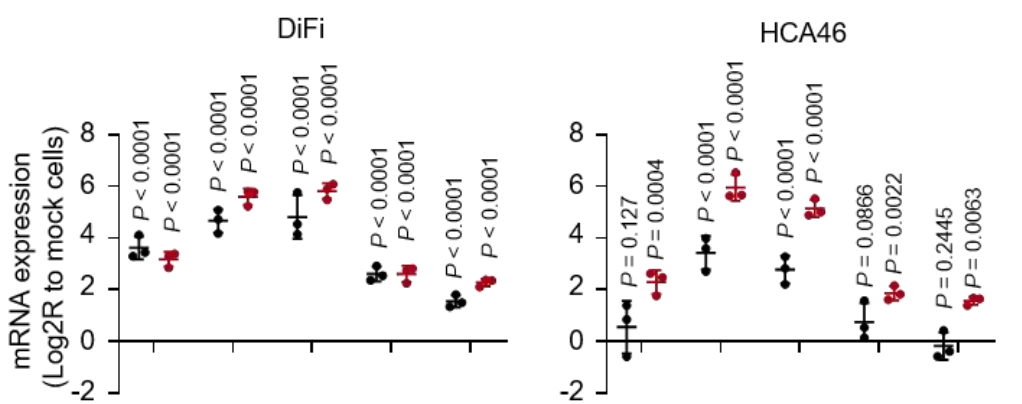

- YAP shRNA-1

- YAP shRNA-2

B

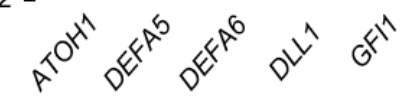

C
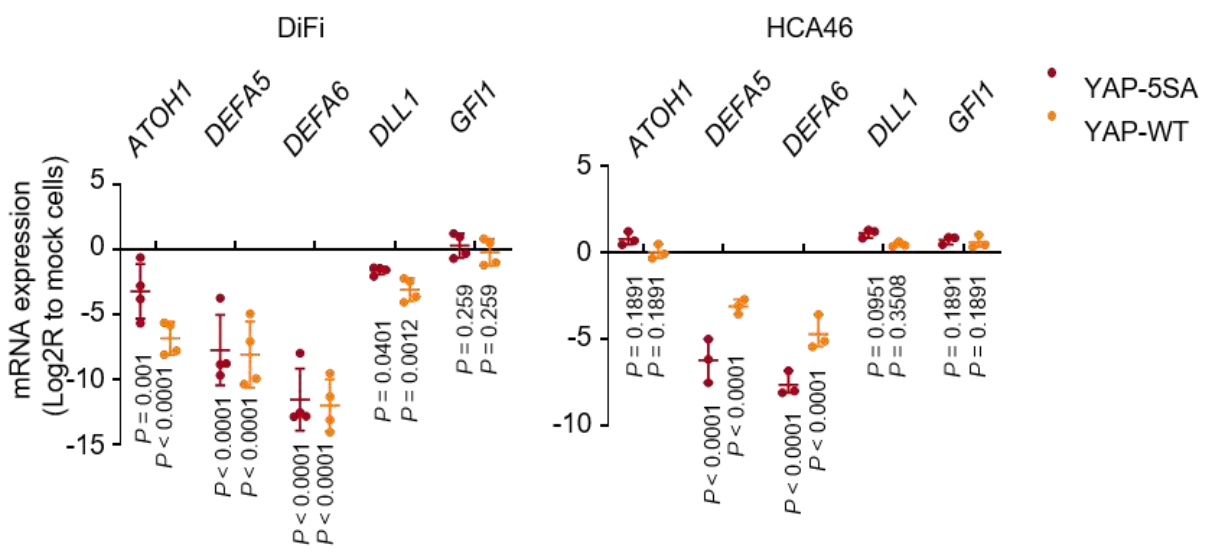

WiFi xenografts

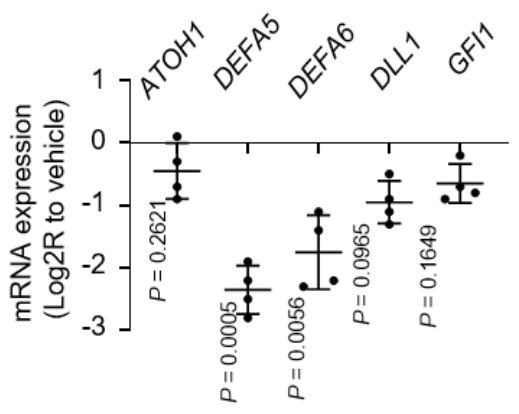

Figure S14. Expression of secretory/Paneth cell genes after YAP silencing or YAP overexpression in vito and in vive

(A) RT-qPCR analysis of the indicated secretory/Paneth-cell genes in WiFi and HCA46 cells after shRNA-based YAP silencing, compared with mock cells. Three independent experiments were performed in technical triplicates. (B) RT-qPCR analysis of the indicated secretory/Paneth-cell genes in WiFi and HCA46 cell lines transduced with YAP-5SA or YAP-WT. Four (DiFi) or 3 (HCA46) independent experiments were performed in technical triplicates. To quantitate gene expression downregulation, technical triplicates consisting of one or two undetermined cycle threshold (Ct) values together with one or two detectable $\mathrm{Ct}>37$ were equalized to a $\mathrm{Ct}$ value of 40 . (C) RTqPCR analysis of the indicated secretory/Paneth-cell genes in established WiFi xenografts 
transduced with doxycycline-inducible YAP-5SA and treated with doxycycline (50 mg/kg daily). Four samples for each condition were analyzed in technical triplicates. In all panels, the plots show means \pm SD. For all experiments, statistical analysis was performed by one-way ANOVA followed by Benjamini, Krieger and Yekutieli FDR correction.

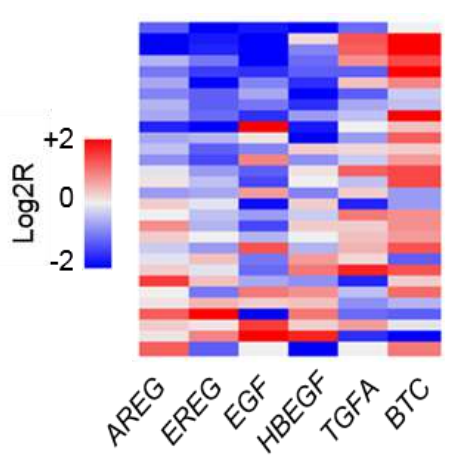

Figure S15. Modulation of EGFR family ligands in PDXs treated with cetuximab

Heatmap showing expression changes for the indicated EGFR ligands in PDXs of the reference collection, after treatment with cetuximab, as assessed by RT-qPCR. Average gene expression, Log2R relative to vehicle-treated tumors: $A R E G-0.28, P=0.206$; $E R E G-0.71, P=0.004$; EGF 0.67, $P=0.349$; HBEGF -0.56, $P=0.220$; TGF $\alpha$ (TGFA) -0.1, $P=0.766$; BTC $0.6, P=0.001$ by two-tailed Wilcoxon test. Benjamini-Hochberg FDR $<0.1$ for EREG and $B T C$. 
A

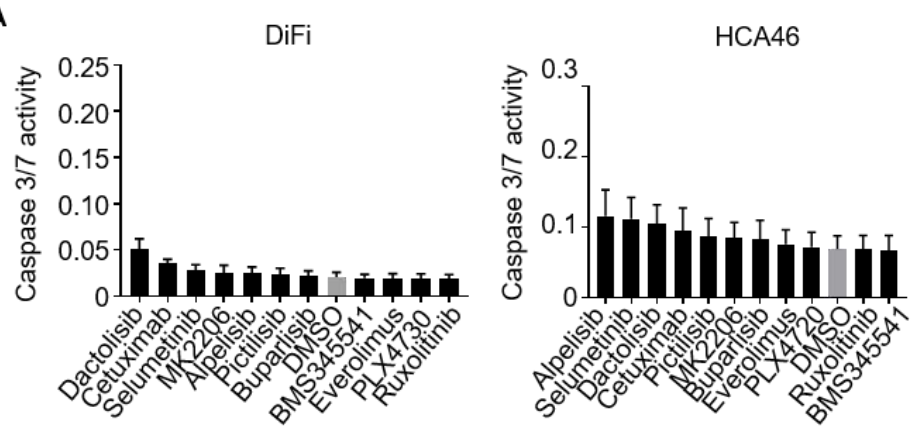

B

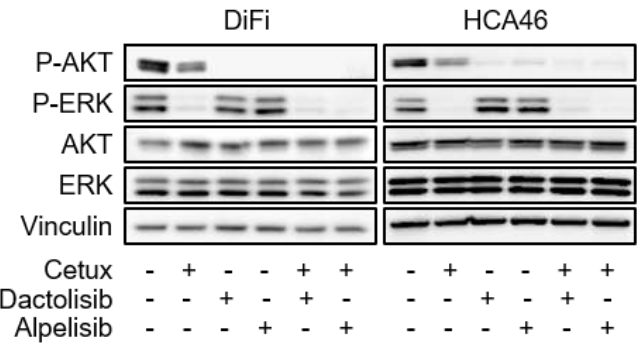

C
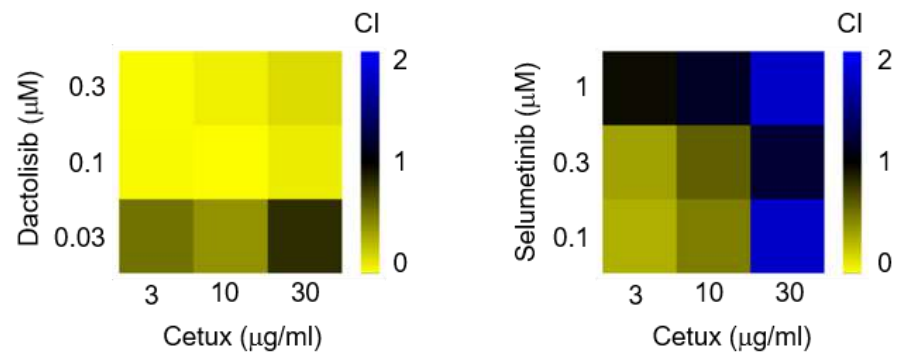

Figure S16. Effects of individual signal inhibition and dual blockade of EGFR and PI3K or EGFR and MEK in CRC cell cultures

(A) Luciferase-based evaluation of apoptosis (assessed by caspase 3/7 activity) in DiFi and HCA46 cell lines either left untreated (vehicle, DMSO, grey bars) or exposed for $24 \mathrm{~h}$ to the indicated drugs, used as monotherapy at the following concentrations: cetuximab, $20 \mu \mathrm{g} / \mathrm{ml}$; BMS345541 (IkB/IKK inhibitor), ruxolitinib (JAK1/JAK2 inhibitor), selumetinib (MEK1 inhibitor), PLX4720 (BRAF inhibitor), alpelisib (PI3Ka inhibitor), $1 \mu \mathrm{M}$; dactolisib (PI3K/mTOR inhibitor), $250 \mathrm{nM}$; everolimus (mTOR inhibitor), $50 \mathrm{nM}$; pictilisib (PI3Ka/ס inhibitor), 100 nM; MK2206 (AKT inhibitor), $0.5 \mu \mathrm{M}$; buparlisib (Pan-PI3K inhibitor), $0.5 \mu \mathrm{M}$. Results represent the means \pm SD of 2 independent experiments performed in biological quintuplicates $(n=10)$. Values shown for cetuximab monotherapy and $\mathrm{Y}$ axis scale are the same as in main Figure 5 for comparative purposes. (B) Western blot analysis of AKT and ERK phosphorylation in DiFi and HCA46 cell lines treated for 2 $\mathrm{h}$ with dactolisib $(250 \mathrm{nM})$, alpelisib $(1 \mu \mathrm{M})$ and/or for $24 \mathrm{~h}$ with cetuximab $(20 \mu \mathrm{g} / \mathrm{ml})$. Total ERK and AKT were used for normalization; vinculin was used as a loading control. Western blots for total proteins were run with the same lysates as those used for anti-phosphoprotein detection but on different gels. The images shown are representative of 2 experiments on independent biological replicates. Cetux, cetuximab. P-AKT, phospho-AKT; P-ERK, phospho-ERK. (C) Analysis of 
combination index $(\mathrm{Cl})$ to assess drug interaction effects in $\mathrm{CRC0078}$ colospheres embedded in PuraMatrix and treated for 2 weeks with the indicated drugs and concentrations. A dose-response matrix design was applied, and $\mathrm{Cl}$ values were calculated by the Compusyn software using the equatorial areas of colospheres as proxies of cell viability. $\mathrm{Cl}>1$ designates antagonism, $\mathrm{Cl}=1$ indicates an additive effect, and $\mathrm{Cl}<1$ is defined as synergy. Cetuximab and dactolisib exhibited a synergistic effect at all concentrations tested $(\mathrm{Cl}$ ranging from 0.007 to 0.822$)$. The synergy between cetuximab and selumetinib occurred only at low compound concentrations and was less pronounced $(\mathrm{Cl}$ ranging from 0,312 to 1,786$)$. 
A

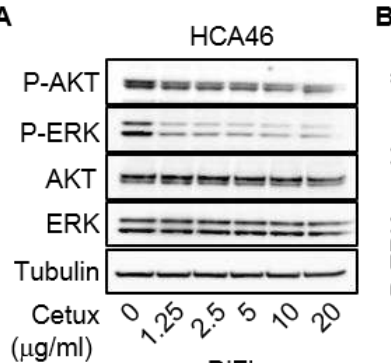

B

$(\mu \mathrm{g} / \mathrm{ml}) \quad \mathrm{DiFi}$

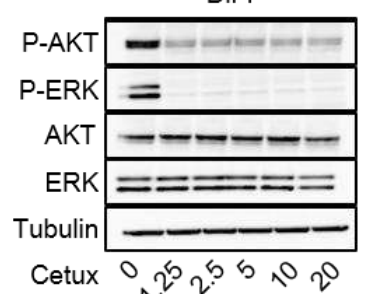

$(\mu \mathrm{g} / \mathrm{ml}) \quad \mathrm{CRC} 0078$
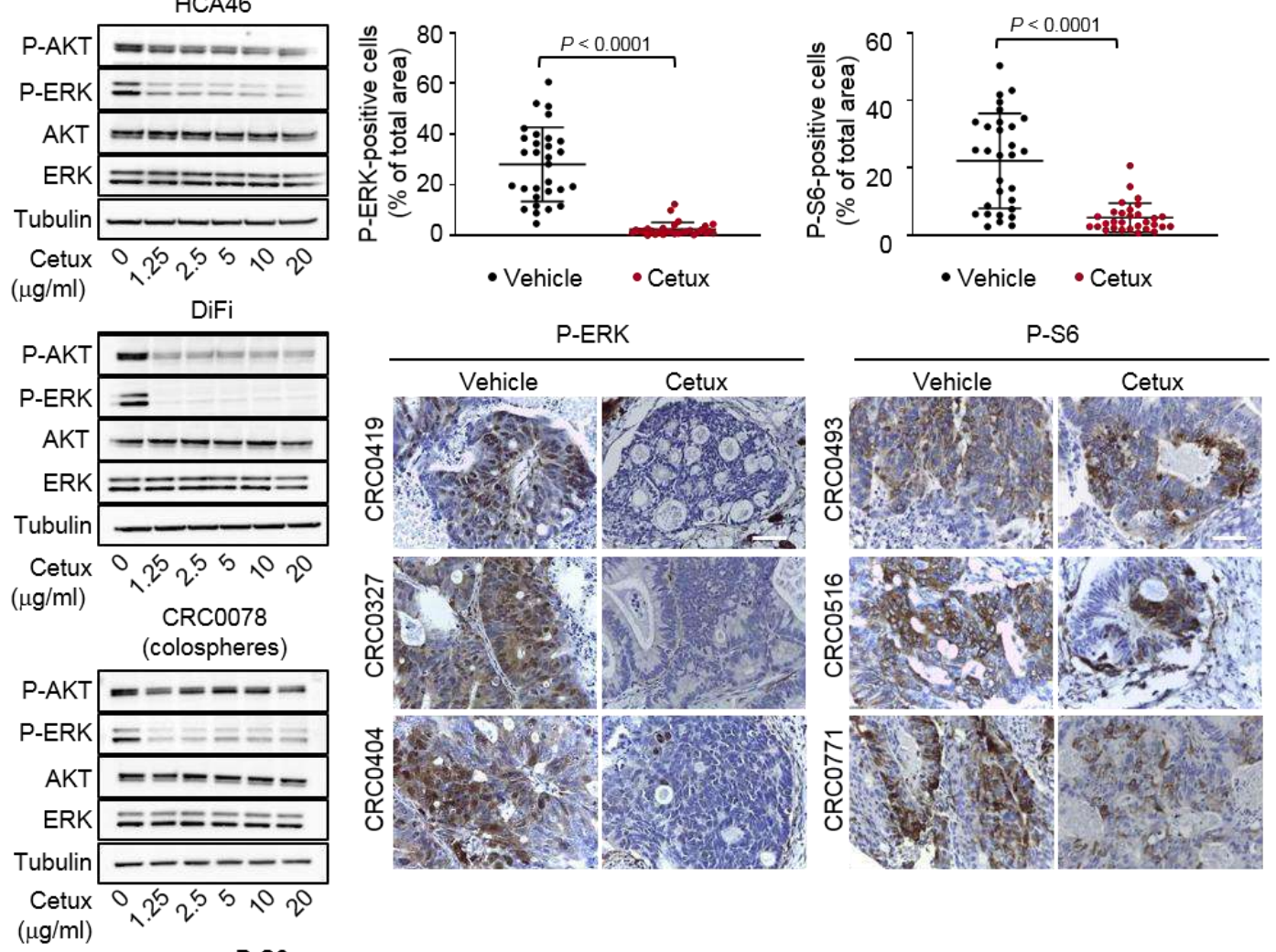
$(\mu \mathrm{g} / \mathrm{ml})$
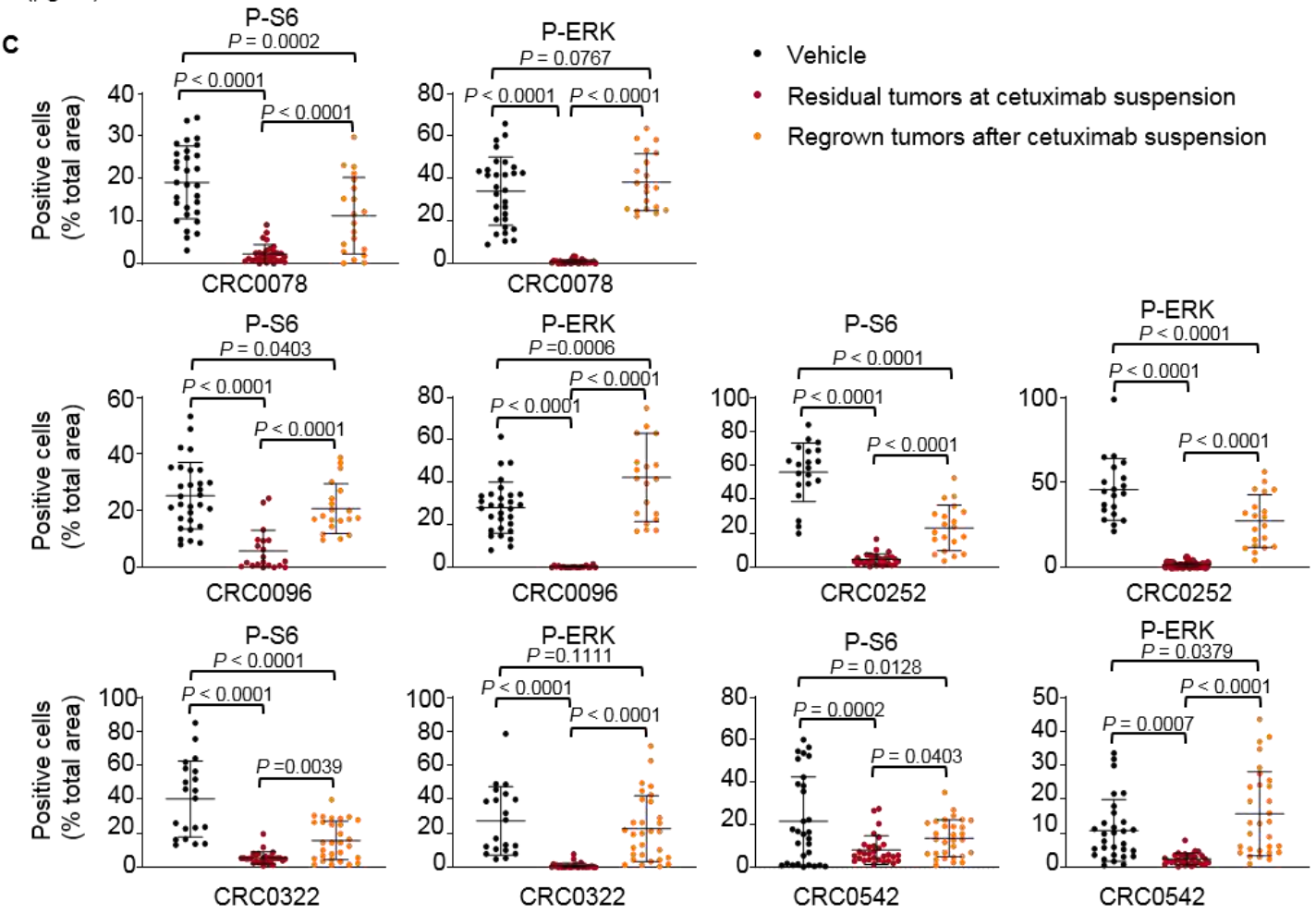

\section{Figure S17. Effects of cetuximab on downstream signals in vitro and in vivo}

(A) Western blot analysis of AKT and ERK phosphorylation in DiFi and HCA46 cell lines and in CRC0078 colospheres treated for $24 \mathrm{~h}$ (cell lines) or $72 \mathrm{~h}$ (CRC0078) with cetuximab at the indicated 
concentrations. Total ERK and AKT were used for normalization; tubulin was used as a loading control. Western blots for total proteins were run with the same lysates as those used for antiphosphoprotein detection but on different gels. The images shown are representative of 2 (DiFi, HCA46) or 3 (CRC0078) experiments on independent biological replicates. Cetux, cetuximab; PERK, phospho-ERK; P-AKT, phospho-AKT. (B) Morphometric quantitation (upper panels) and representative images (lower panels) of phospho-ERK and phospho-S6 immunoreactivity in PDXs from the reference collection after treatment with vehicle (until tumors reached an average volume of $1500 \mathrm{~mm}^{3}$ ) or cetuximab (20 mg/kg twice a week for 6 weeks). Each dot represents the average of 10 optical fields (40X) in a section from randomly chosen tumors from vehicle-treated and cetuximab-treated mice bearing a PDX from the same original patient $(n=30)$. The plots show means \pm SD. Statistical analysis by two-tailed paired Student's $t$-test. Scale bar, $50 \mu \mathrm{m}$. P-S6, phospho-S6. (C) Morphometric quantitation of phospho-S6 and phospho-ERK immunoreactivity in 5 representative PDX models after treatment with vehicle (until tumors reached an average volume of $1500 \mathrm{~mm}^{3}$ ) or cetuximab (20 mg/kg twice a week for 6 weeks). Results are also shown for matched cases after antibody withdrawal, when regrown tumors reached volumes of around 750 $\mathrm{mm}^{3}$. At endpoints, 3 tumors from 3 different mice were explanted and subjected to immunohistochemical analysis. Each dot represents the value measured in one optical field (40x), with 2 to 10 optical fields per tumor depending on the extent of section area ( $n=20$ to 30). The plots show means \pm SD. Statistical analysis by one-way ANOVA followed by Benjamini, Krieger and Yekutieli FDR correction. 
A

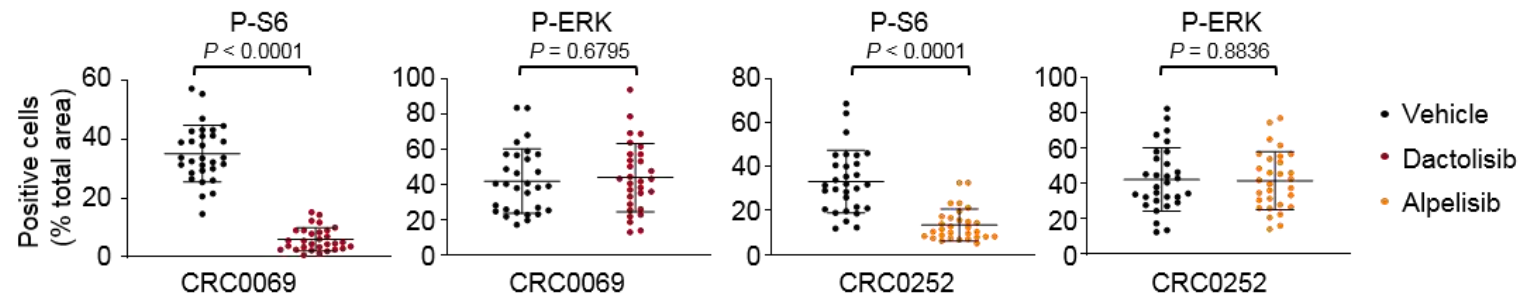

B
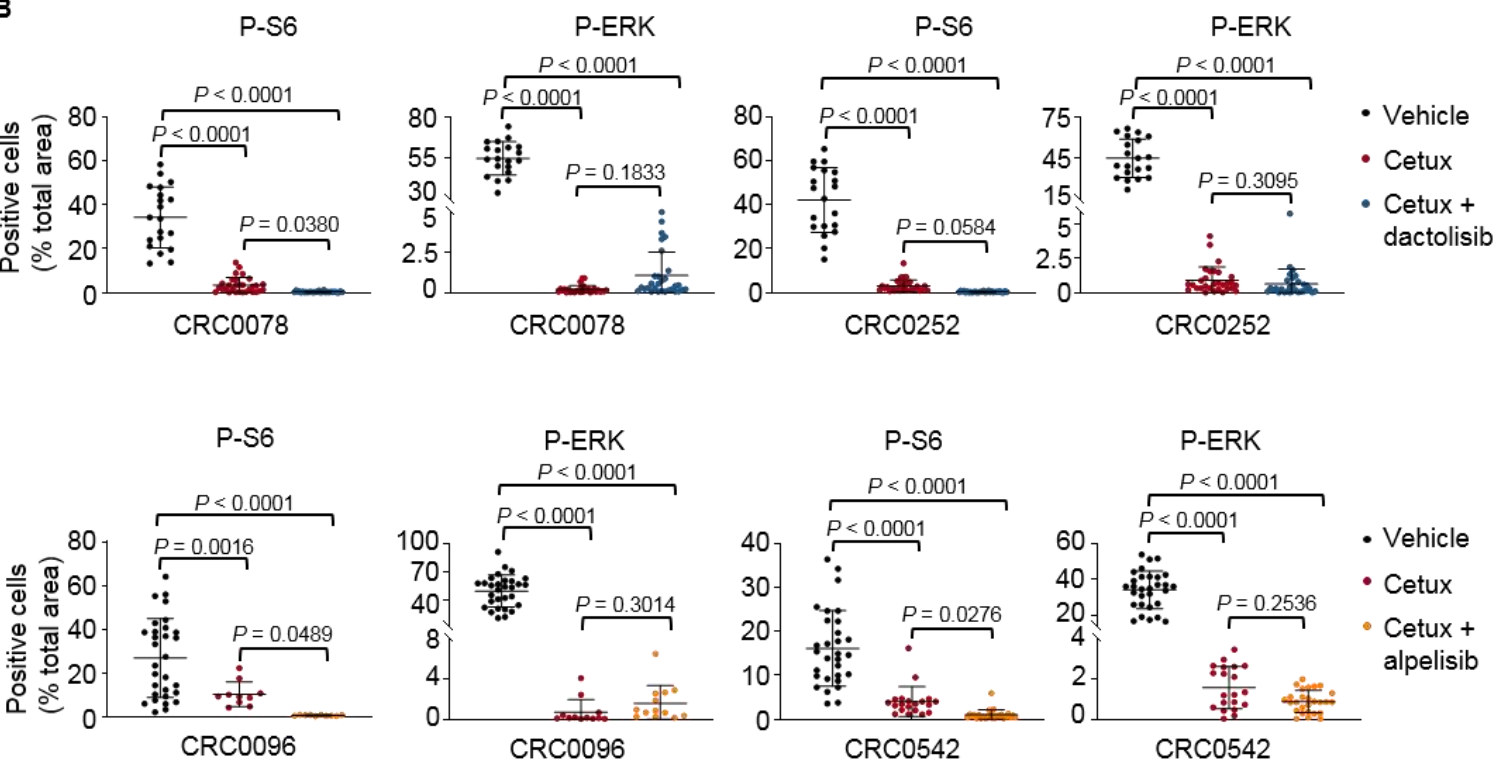

C

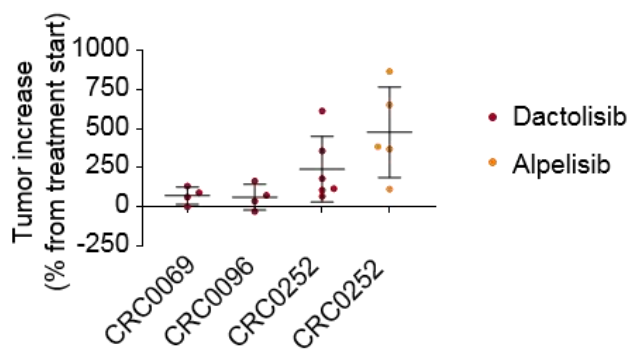

Figure S18. Effects of PI3K inhibitors on downstream signals and tumor growth in vivo

(A) Morphometric quantitation of phospho-S6 and phospho-ERK immunoreactivity in PDXs treated with vehicle (until tumors reached an average volume of $\left.1500 \mathrm{~mm}^{3}\right)$, dactolisib alone $(35 \mathrm{mg} / \mathrm{Kg}$, daily oral gavage) or alpelisib alone (25 $\mathrm{mg} / \mathrm{Kg}$, daily oral gavage) for 4 weeks (CRC0069) or 3 weeks (CRC0252). In the case of CRC0252, the experiment was terminated at 3 weeks because some mice had to be euthanized upon reaching of the humane endpoint. (B) Morphometric quantitation of phospho-S6 and phospho-ERK immunoreactivity in PDXs treated with vehicle (until tumors reached an average volume of $1500 \mathrm{~mm}^{3}$ ), cetuximab alone (4 weeks), cetuximab and dactolisib (4 weeks) or cetuximab and alpelisib (4 weeks). Cetuximab, $20 \mathrm{mg} / \mathrm{Kg}$ (intraperitoneal 
injection twice a week); dactolisib, $35 \mathrm{mg} / \mathrm{Kg}$ (daily oral gavage); alpelisib, $25 \mathrm{mg} / \mathrm{Kg}$ (daily oral gavage). In both (A) and (B), at the end of treatment 3 tumors from 3 different mice (2 tumors from 2 different mice in CRC0096 treated with alpelisib and cetuximab) were explanted and subjected to immunohistochemical analysis. Each dot represents the value measured in one optical field (40x), with 3 to 10 optical fields per tumor depending on the extent of section area ( $n=10$ to 30 ). The plots show means \pm SD. Statistical analysis by two-tailed unpaired Welch's t-test $(\mathbf{A})$ or oneway ANOVA followed by Benjamini, Krieger and Yekutieli FDR correction (B). (C) Tumor volume changes in the indicated cases treated with dactolisib alone or alpelisib alone for 4 weeks (CRC0069, CRC0096) or 3 weeks (CRC0252). Dots represent volume changes of individual mice, and plots show the means \pm SD. $n=4$ to 6 animals per each treatment arm. 

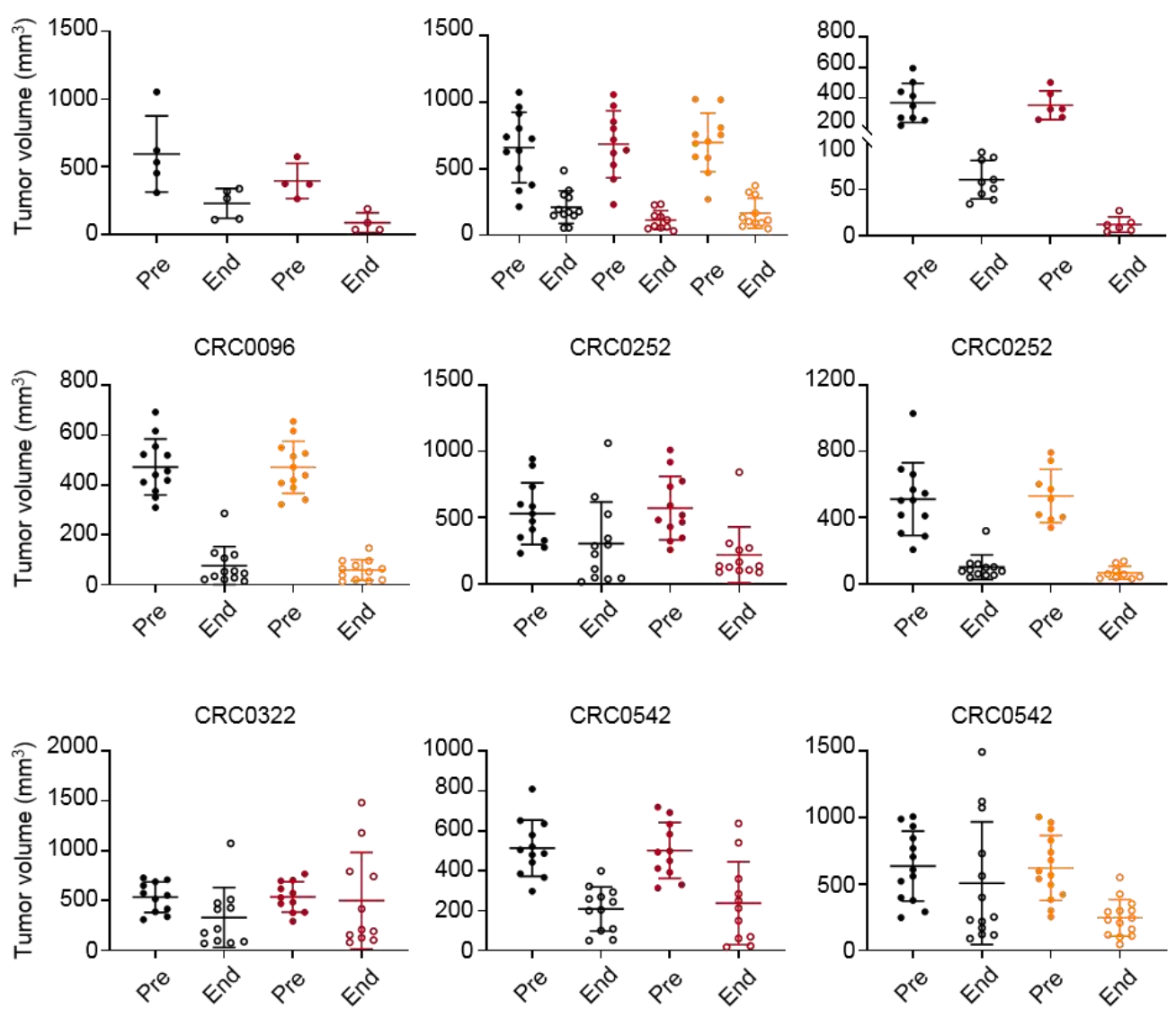

CRC0743

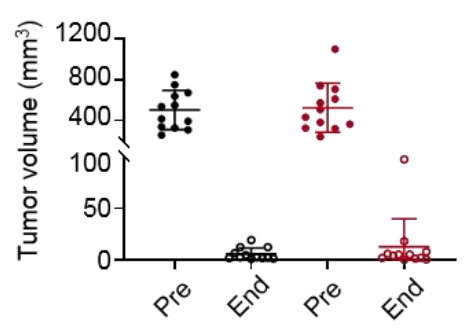

$$
\begin{aligned}
& \text { - Cetux } \\
& \text { - Cetux + dactolisib } \\
& \text { - Cetux + alpelisib }
\end{aligned}
$$

Figure S19. Effects of PI3K inhibition and combined EGFR and PI3K inhibition on MCRC PDX macroscopic residual disease (pre-treatment and end-of-treatment tumor volumes)

Tumor volumes in PDXs treated with the indicated modalities for 4 weeks. Cetuximab, $20 \mathrm{mg} / \mathrm{Kg}$ (intraperitoneal injection twice a week); dactolisib, $35 \mathrm{mg} / \mathrm{Kg}$ (daily oral gavage); alpelisib, 25 $\mathrm{mg} / \mathrm{Kg}$ (daily oral gavage). Dots represent tumor volumes in individual mice, and plots show the means \pm SD for each treatment arm. $n=4$ to 14 animals per each treatment arm. Cetux, cetuximab; Pre, pre-treatment (day 0); End, end-of-treatment (4 weeks). 
A

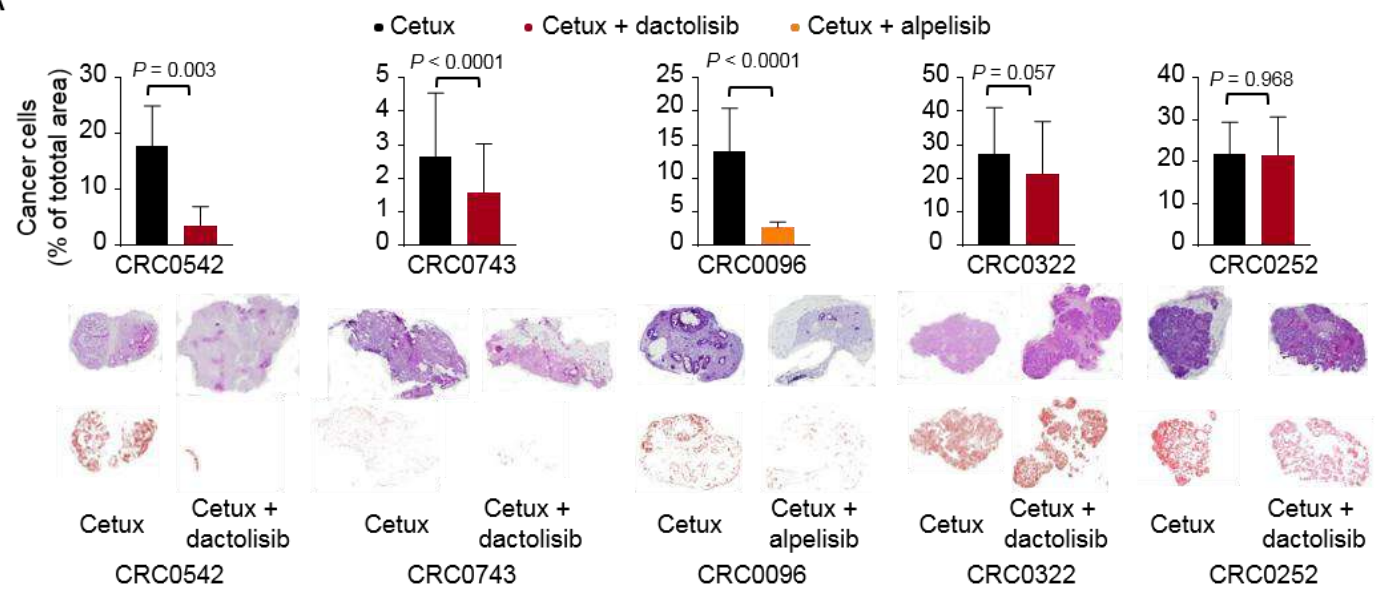

B
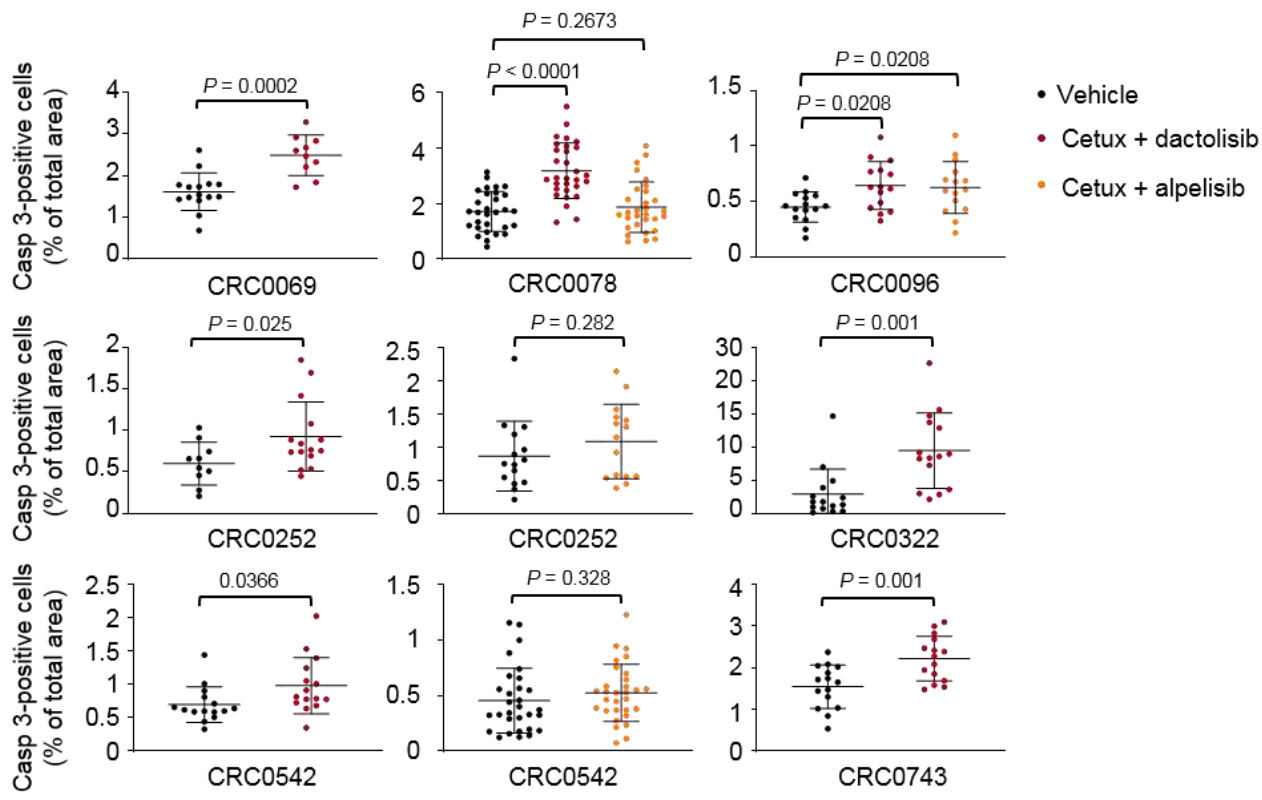

C
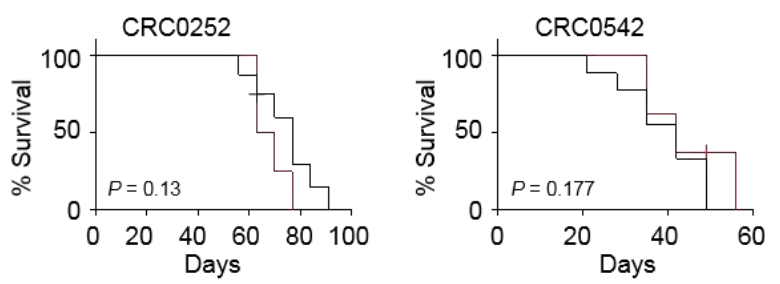

- Cetux

- Cetux + dactolisib

- Cetux + alpelisib
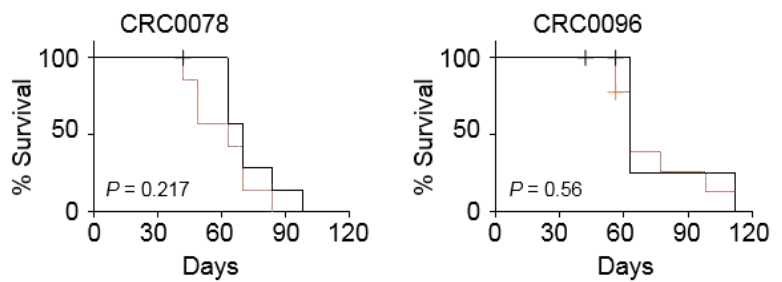
Figure S20. Effects of combined EGFR and PI3K inhibition on mCRC PDX microscopic residual disease, apoptosis, and survival

(A) Microscopic assessment of residual cancer cell burden in PDXs treated with the indicated modalities for 4 weeks. Cetuximab, $20 \mathrm{mg} / \mathrm{Kg}$ (intraperitoneal injection twice a week); dactolisib, $35 \mathrm{mg} / \mathrm{Kg}$ (daily oral gavage); alpelisib, $25 \mathrm{mg} / \mathrm{Kg}$ (daily oral gavage). Upper panels indicate morphometric quantitations ( $n=4$ to 89 depending on the extent of section area); lower panels include hematoxylin-and-eosin staining and visualization of cancer cells (in brown) by digital segmentation. Statistical analysis by two-tailed unpaired Welch's t-test. Cetux, cetuximab. (B) Morphometric quantitation of apoptosis (caspase 3 staining) in PDXs treated for 24 hours with the indicated modalities. After treatment, 3 tumors from 3 different mice were explanted and subjected to immunohistochemical analysis. Each dot represents the value measured in one optical field (20x), with 3 to 10 optical fields per tumor depending on the extent of section area ( $n=10$ to 30 ). The plots show means \pm SD. For CRC0069, CRC0252, CRC0322, CRC0542, and CRC0743, statistical analysis was performed by two-tailed unpaired Welch's $t$-test. For CRC0096 and CRC0078, statistical analysis was performed by one-way ANOVA followed by Benjamini, Krieger and Yekutieli FDR correction. Casp, caspase. (C) Kaplan-Meier survival curves in PDXs after discontinuation of the indicated treatments. $n=6$ to 9 animals per each treatment arm. Statistical analysis by Log-rank (Mantel-Cox) test. 
A
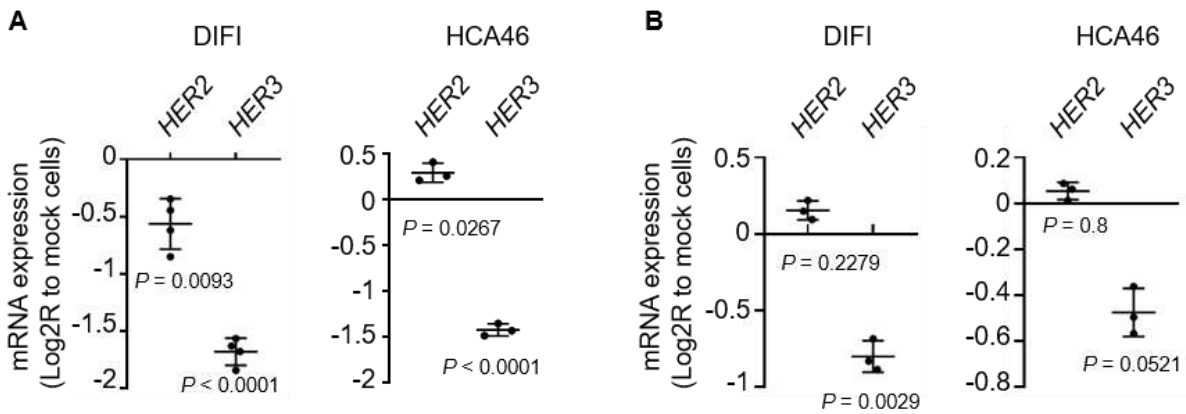

YAP-5SA

YAP-WT

C
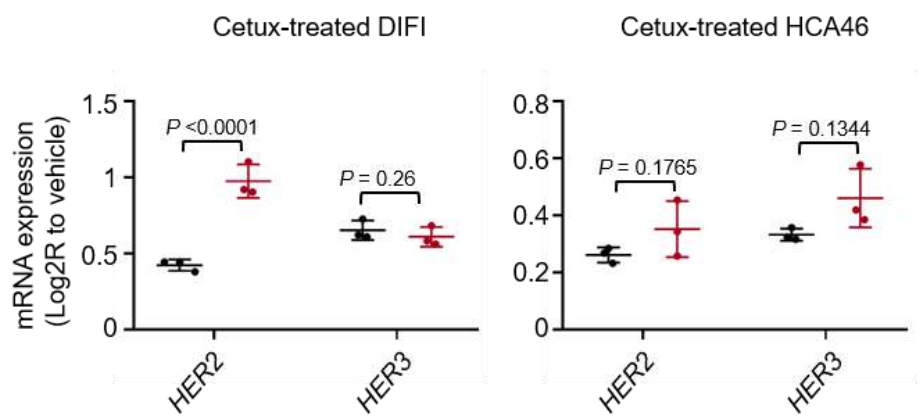

- Mock

- YAP-WT

D

DiFI

HCA46
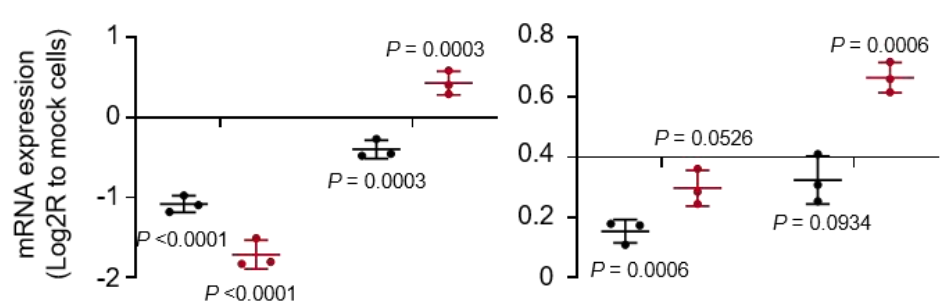

- YAP shRNA-1

- YAP shRNA-2<smiles>[C]1=CC=C1</smiles><smiles>c1ccccc1</smiles><smiles>[C]=[Co]</smiles><smiles>C1=CCCCCC1</smiles>

Figure S21. YAP-dependent transcriptional modulation of $H E R 2$ and $H E R 3$ in CRC cell lines (A and B) RT-qPCR analysis of HER2 and HER3 transcript expression in DiFi and HCA46 cell lines transduced with constitutive active YAP (YAP-5SA) (A) or wild-type YAP (YAP-WT) (B). (C) RT-qPCR analysis of HER2 and HER3 transcript expression in DiFi and HCA46 cell lines transduced with a pLVX-control vector (mock) or wild-type YAP (YAP-WT), and treated with cetuximab $(20 \mu \mathrm{g} / \mathrm{ml})$ for $72 \mathrm{~h}$. (D) RT-qPCR analysis of HER2 and HER3 transcript expression in DiFi and HCA46 cells after shRNA-based YAP silencing, compared with mock cells. In all panels, the plots show means \pm SD. In panel $(\mathbf{A}), 4$ (DiFi) or 3 (HCA46) independent experiments were performed in technical triplicates. In all other panels, 3 independent experiments were performed in technical triplicates. Statistical analysis was performed by one-way ANOVA followed by Benjamini, Krieger and Yekutieli FDR correction. 


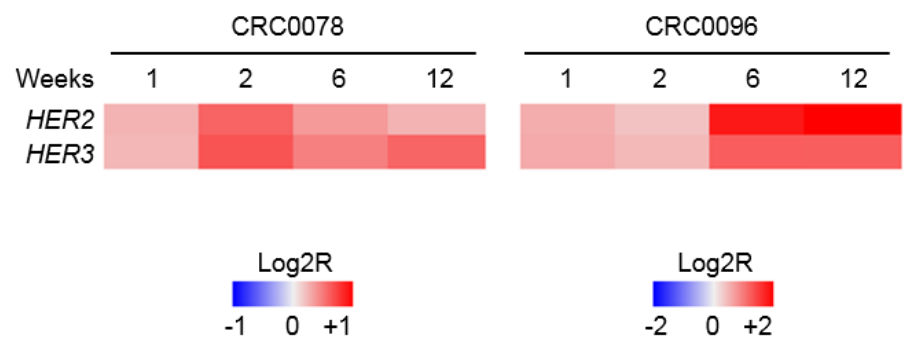

Figure S22. Modulation of HER2 and HER3 expression in PDXs during prolonged treatment with cetuximab

Heatmap showing expression changes for the indicated secretory/Paneth cell markers in 2 representative PDXs treated with cetuximab $(20 \mathrm{mg} / \mathrm{kg}$ twice a week intraperitoneally) and monitored longitudinally for 12 weeks. At the indicated times, 1 tumor from 1 mouse was explanted and subjected to RT-qPCR analysis. 

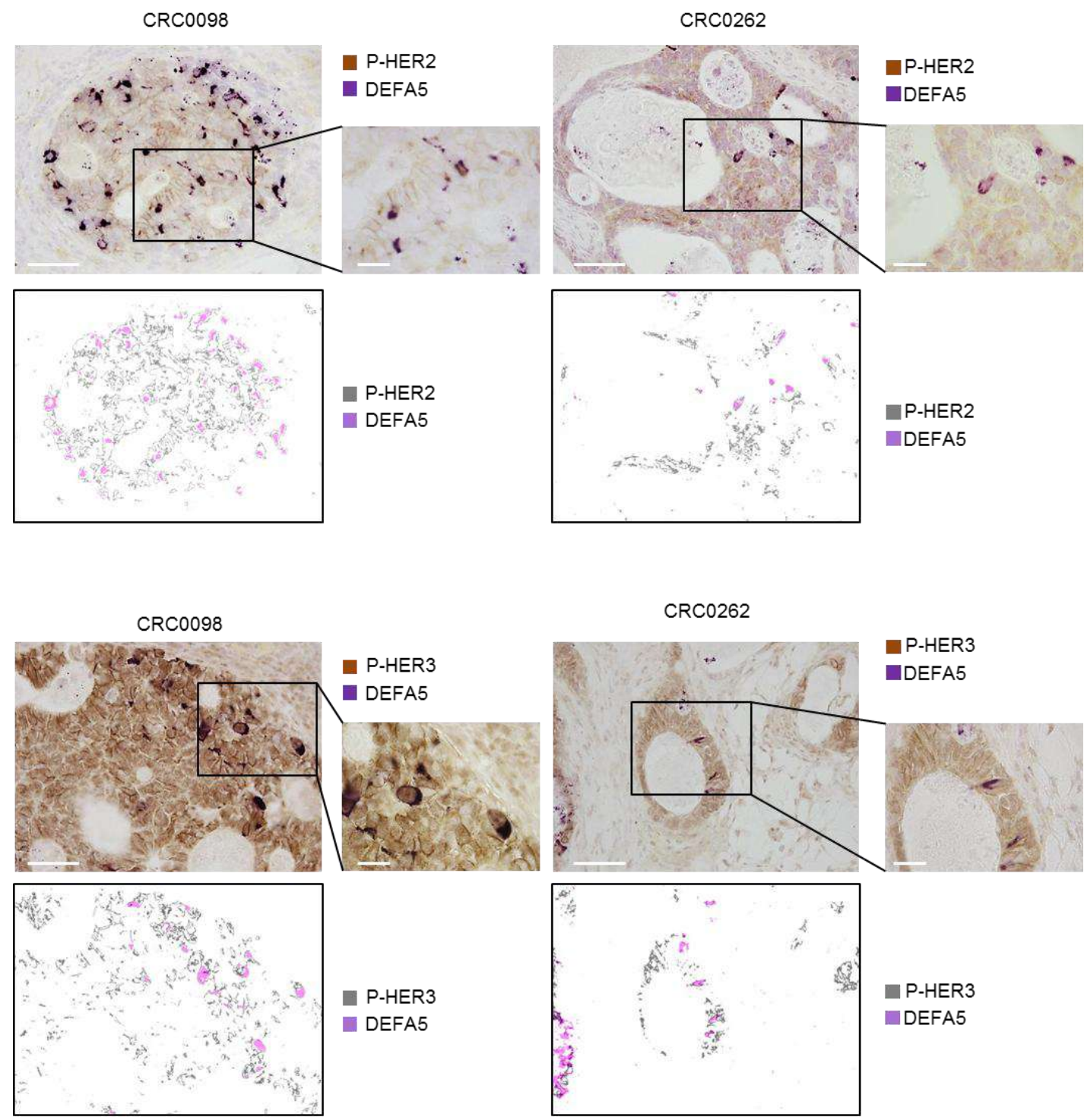

Figure S23. DEFA5 and HER2/HER3 double staining in representative mCRC PDXs treated with cetuximab

HER2 or HER3 and $\beta$-catenin double staining in 2 PDX models treated with cetuximab $(20 \mathrm{mg} / \mathrm{kg}$ twice a week intraperitoneally) for 6 weeks. For each model, the upper panels are representative images of bright-field optical sections. The lower panels show the corresponding color deconvolution segmentation. Scale bar, $50 \mu \mathrm{M}$ (insets, $20 \mu \mathrm{M}$ ). P-HER2, phoshpo-HER2; P-HER3, phospho-HER3. 


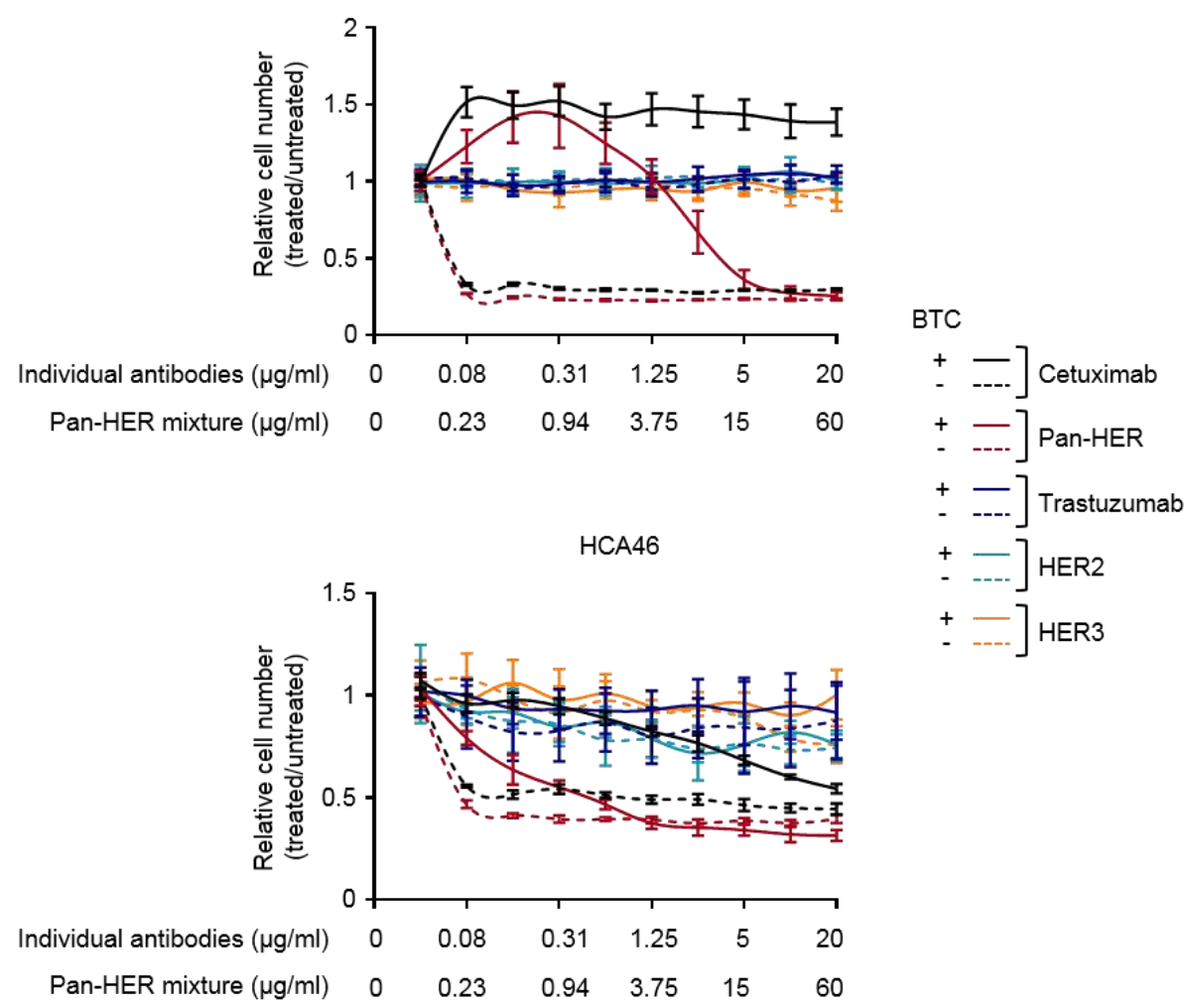

Figure S24. CRC cell line sensitivity to individual targeting of HER family members

Quantitation of cell number (assessed by ATP content) in DiFi and HCA46 cell lines treated for $72 \mathrm{~h}$ with the indicated antibodies at the indicated concentrations in the absence or presence of $10 \mathrm{ng} / \mathrm{ml}$ BTC. Results represent the means \pm SD of 2 independent experiments performed in biological triplicates $(n=6)$. HER2, anti-HER2 isolated component of Pan-HER; HER3, anti-HER3 isolated component of Pan-HER. 


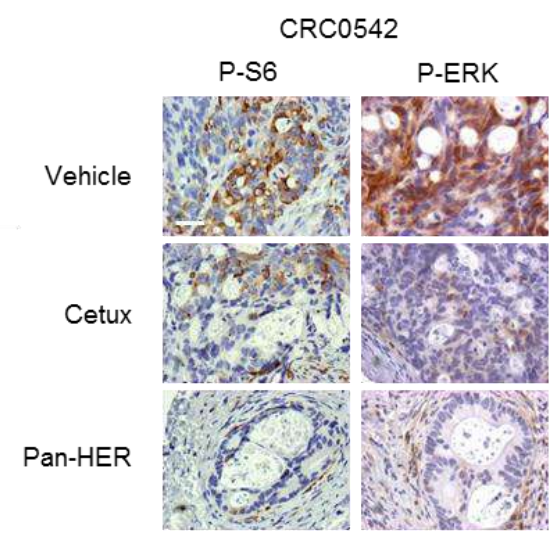

Figure S25. Effects of cetuximab and Pan-HER on EGFR downstream targets in vivo

Representative images (right panels) of phospho-S6 and phospho-ERK immunoreactivity (see main Figure 7 for quantitations) in PDXs after treatment with vehicle (until tumors reached an average volume of $1500 \mathrm{~mm}^{3}$ ), cetuximab (20 mg/Kg, intraperitoneal injection twice a week for 5 weeks), or Pan-HER (60 mg/Kg, intraperitoneal injection twice a week for 5 weeks). At the end of treatment, 3 tumors from 3 different mice were explanted and subjected to immunohistochemical analysis. Cetux, cetuximab; P-S6, phospho-S6; P-ERK, phospho-ERK. Scale bar, $50 \mu \mathrm{m}$. 